TITLE:

\title{
Nongeminate charge recombination in organic photovoltaics
}

\section{AUTHOR(S):}

Fukuhara, Tomohiro; Tamai, Yasunari; Ohkita, Hideo

\section{CITATION:}

Fukuhara, Tomohiro ...[et al]. Nongeminate charge recombination in organic photovoltaics. Sustainable Energy \& Fuels 2020, 4(9): 4321-4351

ISSUE DATE:

2020-09-01

URL:

http://hdl.handle.net/2433/254684

\section{RIGHT:}

This is the accepted Manuscript of the article, which has been published in final form at

http://doi.org/10.1039/d0se00310g.; The full-text file will be made open to the public on $24 \mathrm{June} 2021$ in accordance with publisher's 'Terms and Conditions for Self-Archiving'; ここの論文は出版社版でありません。引用の際には出版社版 をご確認ご利用ください。; This is not the published version. Please cite only the published version. 


\title{
ARTICLE
}

\section{Nongeminate Charge Recombination in Organic Photovoltaics}

Received 00th January 20xx, Accepted 00th January 20xx DOI: $10.1039 / x 0 x \times 00000 x$

\author{
Tomohiro Fukuhara, ${ }^{a}$ Yasunari Tamai, ${ }^{* a, b}$ and Hideo Ohkita*a
}

\begin{abstract}
Charge recombination is a major loss process in state-of-the-art organic photovoltaics (OPVs). Nongeminate recombination (NGR) is typically in competition with collection of photogenerated charge carriers to each electrode not only at the shortcircuit but also at the open-circuit conditions, and therefore impacts on both external quantum efficiency (EQE) and fill factor (FF). In most cases, therefore, the optimised active layers are kept as thin as possible (typically $\sim 100 \mathrm{~nm}$ ) to avoid NGR loss although it is not thick enough to absorb the solar light effectively. As such, a key to efficient EQE and FF is suppressing NGR beyond limitations arising from the Langevin recombination. In addition, NGR is closely related to open-circuit voltage ( $\left.V_{0 c}\right)$ as well. In particular, nonradiative NGR is a critical loss process in OPVs. In this review, we aim to summarise our current understanding of underlying photophysics that governs NGR in OPVs. Recent experimental results and proposed mechanisms relevant to NGR are summarised where we highlight the significant importance of morphology and energetics for suppressing NGR. These insights will provide a comprehensive understanding of the recombination and future materials/devices design principles.
\end{abstract}

\section{Introduction}

Organic photovoltaics (OPVs) based on bulk-heterojunction (BHJ) active layers consisting of electron-donating semiconducting polymers and electron-accepting molecules are gaining interest because of their promising advantages of low cost, light weight, and flexibility. Over the past two decades, the power conversion efficiency (PCE) has increased from $\sim 2$ to $>18 \% .{ }^{1-13}$ In order to further improve PCE toward 20\%, the external quantum efficiency (EQE) and fill factor (FF) of single-junction OPVs likely need to approach $90 \%$ and 0.75 , respectively, as shown in Figure 1 . Here we assumed the photon energy (voltage) loss defined as $E_{\mathrm{g}}-q V_{\mathrm{oc}}$ to be $0.5 \mathrm{eV}$ where $E_{\mathrm{g}}, q$, and $V_{\mathrm{OC}}$ are the bandgap energy of the material, elementary charge, and open-circuit voltage, respectively. Although several OPVs with an FF of $>0.75$ have already been reported,, 14 achieving such a high FF and EQE simultaneously is undoubtedly a challenging issue. To achieve an EQE of $90 \%$, the active layer must absorb more than $90 \%$ of incident photons above $E_{\mathrm{g}}$ as the EQE never exceeds the photon absorption efficiency (unless including multi-excitons generation such as singlet fission). Thus, the active layer must be $>200 \mathrm{~nm}$ in thickness. However, increasing active layer thickness usually degrades device performance especially FF because charge carriers must travel further in the active layer, and hence are more likely to recombine before reaching the electrodes, indicating that there is a trade-off between EQE and FF. Thus, slowing charge recombination while maintaining charge mobility is important to achieve a high FF and EQE simultaneously.

\footnotetext{
a. Department of Polymer Chemistry, Graduate School of Engineering, Kyoto University, Katsura, Kyoto 615-8510, Japan.

b. PRESTO, Japan Science and Technology Agency, 4-1-8 Honcho, Kawaguchi, Saitama, 332-0012, Japan.
}

Another challenging issue is to minimise the photon energy (voltage) loss. In Figure 1, the photon energy loss is assumed to be $0.5 \mathrm{eV}$. However, the photon energy loss has been typically reported to be as large as more than $0.7 \mathrm{eV}$ for most OPVs. A part of this photon energy loss arises during the charge generation process. Because excitons in OPVs split to form charge transfer state (CTS) with the aid of the offset in molecular orbital energy levels as summarised in the next section, this photon energy loss was thought to be

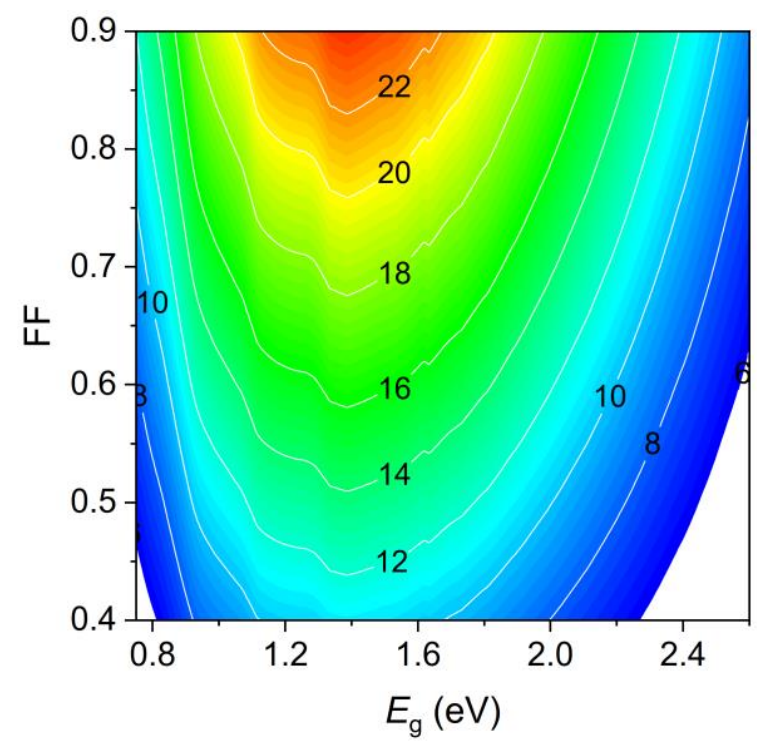

Figure 1. Contour plot showing the calculated PCE against the bandgap energy $E_{\mathrm{g}}$ and FF. The $q V_{\mathrm{OC}}$ and EQE were fixed to $E_{\mathrm{g}}-$ $0.5 \mathrm{eV}$ and $90 \%$, respectively. The $J_{S C}$ was calculated by integrating a product of solar photon flux under AM1.5G condition and the rectangle EQE spectrum. 
unavoidable. ${ }^{15}$ Recent studies, however, have shown that the photon energy loss due to charge generation can be suppressed to less than $0.1 \mathrm{eV}$, resulting in a voltage loss of less than $0.6 \mathrm{~V}$ for state-of-theart devices. ${ }^{16-19}$ The other source of voltage loss is due to charge recombination and the consequent decrease in quasi-Fermi level splitting. At this moment, there are only limited papers reporting OPVs with a voltage loss of less than $0.5 \mathrm{~V}^{20-24}$ In other words, there is still room for further improvement in $V_{O C}$ as will be discussed later. Therefore, suppressing charge recombination is of ultimate importance to further improve not only FF but also $V_{O C}$.

Significant progress has been made in the development of new materials as well as our understanding of the basic photophysical phenomena in OPVs. ${ }^{25-37}$ Yet many questions about charge recombination still remain unclear or controversial. Our motivation in this article is to give a brief introduction of underlying photophysics that governs nongeminate recombination (NGR), also referred to as bimolecular recombination. This review consists of three parts. First, we summarise the fundamentals of photophysics and charge recombination in OPVs. Then, we provide a brief explanation for typical experimental techniques to evaluate the recombination dynamics. Finally, we highlight recent researches on reduced NGR, the origin of slower NGR, and the relevance to photovoltaic parameters.

\section{Fundamental Photophysics in OPVs}

Upon photoexcitation, as shown in Figure 2, singlet excitons are promptly generated in either the electron donor or acceptor material in the active layer and then diffuse to a donor/acceptor interface. Because of the short lifetime of singlet excitons, typically less than 1 $\mathrm{ns}^{38}$ the BHJ concept has been widely applied to OPVs where donor and acceptor materials are finely phase-separated with a domain size of less than $10 \mathrm{~nm}^{39,40}$ As a result of CT at the interface, singlet excitons separate into holes on the donor and electrons on the acceptor. If the hole and electron spatially separate further, they would dissociate into charge carriers free from Coulomb attraction, which can survive on a time scale of ns- $\mu$ s. Otherwise, the hole and electron pair is likely to recombine monomolecularly to the ground state (GS). This is called geminate recombination (GR), which is monomolecular recombination of an electron-hole pair generated upon photoexcitation. As reported previously, GR is one of the most detrimental processes in OPVs with modest efficiencies. ${ }^{41-43}$ On the other hand, some high-efficiency OPVs exhibit excellent charge (a)

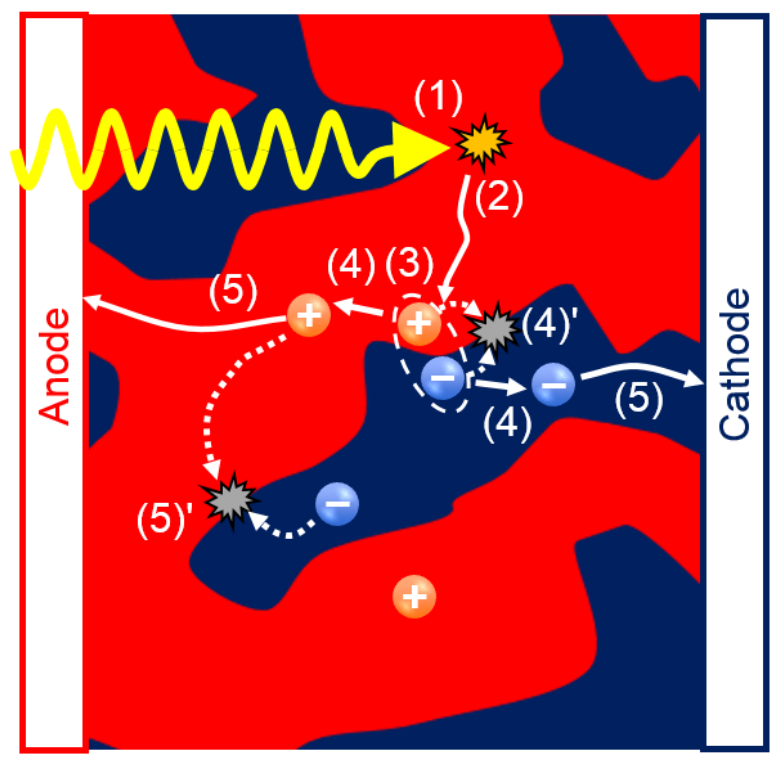

(b)

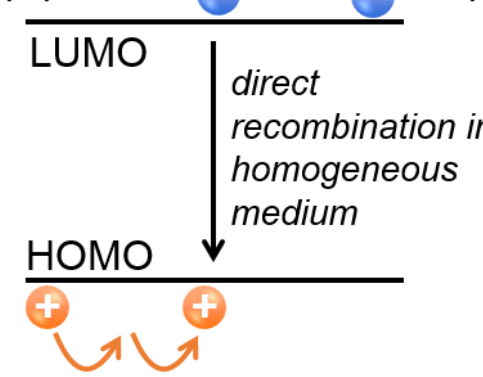

Langevin recombination SRH recombination

(d)

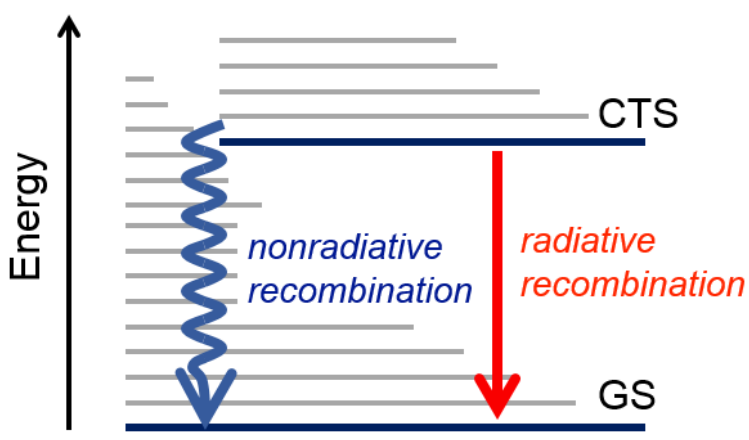

Figure 2. (a) Bulk heterojunction OPVs under light illumination. Light to electricity conversion undergoes taking five fundamental steps: (1) photon absorption to form excitons, (2) exciton diffusion to the interface, (3) charge transfer at the interface, (4) charge dissociation into free carriers, and (5) charge collection to each electrode. (b) Langevin recombination between free charges in homogeneous medium. (c) Shockley-Read-Hall (SRH) recombination between free holes (or electrons) and deeply trapped electrons (or holes). (d) Radiative and nonradiative transitions of charge transfer state (CTS) to the ground state (GS). (4)' Geminate recombination (GR) is monomolecular recombination of a bound charge pair at the interface (3) in the panel (a) generated upon photoexcitation. (5)' Nongeminate recombination (NGR) is bimolecular recombination of free charge carriers generated after the charge dissociation (4) in the panel (a). Both GR and NGR deactivate to the GS radiatively or nonradiatively as shown in the panel (d). 
dissociation efficiency overcoming GR on a sub-ps time scale. ${ }^{44-51}$ This results in extremely high internal quantum efficiencies under short-circuit conditions, approaching unity in some blend systems. ${ }^{52-}$ ${ }^{54}$ Although GR is still a key issue in our research field, we would not focus on the competition between charge dissociation and GR in this review. After the dissociation, free charge carriers are then transported in the active layer to each electrode to be collected as photocurrent. As most OPVs comprise nanoscale phase-separated domains, holes and electrons frequently encounter at the interface during charge transport, and then recombine bimolecularly. This is called NGR, which is bimolecular recombination of free charges generated after charge dissociation. Both GR and NGR deactivate to the GS radiatively or nonradiatively. As mentioned above, GR loss is not significant in the state-of-the-art efficient OPVs, NGR should be the dominant loss process.

\section{Fundamentals of NGR}

If the oppositely charged carriers are statistically independent of each other, then carrier recombination is a random process and hence depends on charge carrier densities and the relative mobility $\mu\left(\mu=\mu_{\mathrm{e}}+\mu_{\mathrm{h}}\right.$ where $\mu_{\mathrm{e}}$ and $\mu_{\mathrm{h}}$ are electron and hole mobility, respectively). Thus, the recombination rate $R$ can be described as follows.

$$
R=-\frac{\mathrm{d} n}{\mathrm{~d} t}=\gamma\left(n p-n_{\mathrm{i}} p_{\mathrm{i}}\right) \approx \gamma n p
$$

Here, $n$ and $p$ represent charge carrier density for electrons and holes, respectively. The prefactor $\gamma$ is the recombination rate coefficient and is a function of the relative mobility $\mu$. For simplicity, we will disregard intrinsic carrier density $n_{\mathrm{i}}$ and $p_{\mathrm{i}}$ as $n_{\mathrm{i}} p_{\mathrm{i}}<<n p$ under 1 sun condition.

The Coulomb capture radius $r_{c}$, at which the Coulomb attraction between opposite charges is equal to the thermal energy from surroundings, is defined as

$$
r_{\mathrm{c}}=\frac{q^{2}}{4 \pi \varepsilon_{0} \varepsilon_{\mathrm{r}} k_{\mathrm{B}} T}
$$

where $q, \varepsilon_{0}, \varepsilon_{\mathrm{r}}, k_{\mathrm{B}}$, and $T$ are the elementary charge, permittivity of vacuum, relative permittivity, Boltzmann constant, and absolute temperature, respectively. At room temperature, this value can be as large as $>10 \mathrm{~nm}$ because of the low dielectric constant of organic semiconductors $\left(\varepsilon_{\mathrm{r}} \approx 3-4\right)$, meaning that charges always feel the Coulomb interaction in the active layer because the domain size of $\mathrm{BHJ}$ type OPVs is in the same order as mentioned above. This indicates that charges easily recombine bimolecularly. By considering entropy contribution to the free energy, 41,42 it would be reduced to a few $\mathrm{nm}$. The entropy effect on NGR will be discussed later.

\section{Reaction Order}

The reaction order $\Delta$ evaluated by steady-state $J-V$ measurements describes how the recombination rate $R$ scales with the free charge carrier density. In the case of Langevin NGR, the reaction order $\Delta$ is 2. On the other hand, in the case of Shockley-Read-Hall (SRH) recombination, ${ }^{55,56}$ where mobile free charge carriers recombine with opposite charges in deep traps, the reaction order is 1 because the density of deeply trapped charges does not change. In real cases in which both NGR and SRH recombination contribute to charge dynamics, $\Delta$ is expected to be in between 1 and 2 .

On the other hand, the empirical reaction order $\delta$ evaluated by transient measurements described later is often higher than 2 . Note that while the Auger recombination is known as a third-order recombination process, ${ }^{57}$ it is not the case for OPVs because it needs a much higher charge density than that under 1 sun illumination. The empirical $\delta$ higher than 2 is usually explained by the presence of an energetic disorder leading to a broad electronic density of states. ${ }^{58}$ This leads to a discrepancy between the $\Delta$ and $\delta$ because the former is based on free charges and the later on all charges in a device including both free and trapped charges. Kirchartz and Nelson have considered the recombination of free charge carriers with trapped charge carriers in an exponential tail state to explain the link between $\Delta$ and $\delta$, and also the relation between $\delta$ and the diode ideality factor $n_{\text {id }}$ as follows. ${ }^{59}$ Since $\delta$ is defined as $R \propto n^{\delta}$,

$$
\delta=\frac{\mathrm{d} \ln R}{\mathrm{~d} \ln n}=\frac{\mathrm{d} V}{\mathrm{~d} \ln n} \cdot \frac{\mathrm{d} \ln R}{\mathrm{~d} V}
$$

$R$ and $n$ are expressed as

$$
\begin{gathered}
R=R_{0} \exp \left(\frac{q V}{n_{\mathrm{id}} k_{\mathrm{B}} T}\right) \\
n=n_{0} \exp \left(\frac{q V}{m k_{\mathrm{B}} T}\right)
\end{gathered}
$$

where $m$ is a constant which determines the slope of $n$ against voltage. Then, $\delta$ is expressed as

$$
\delta=\frac{m}{n_{\mathrm{id}}}
$$

On the other hand, $\Delta$ is defined as $R \propto n_{\mathrm{f}} A$,

$$
\Delta=\frac{\mathrm{d} \ln R}{\mathrm{~d} \ln n_{\mathrm{f}}}=\frac{\mathrm{d} V}{\mathrm{~d} \ln n_{\mathrm{f}}} \cdot \frac{\mathrm{d} \ln R}{\mathrm{~d} V}
$$

The density of free electrons $n_{\mathrm{f}}$ (holes $p_{\mathrm{f}}$ ) is described as

$$
n_{\mathrm{f}}\left(p_{\mathrm{f}}\right) \propto \exp \left(\frac{q V}{2 k_{\mathrm{B}} T}\right)
$$

Thus, $\Delta$ is expressed as

$$
\Delta=\frac{2}{n_{\mathrm{id}}}
$$

When we assume recombination between free charges, we obtain $n$ $=n_{\mathrm{f}}, p=p_{\mathrm{f}}$, and $m=2$ by comparing Equation 5 with Equation 8 . Thus, $R$ is described as

$$
R \propto n_{\mathrm{f}} p_{\mathrm{f}} \propto \exp \frac{q V}{k_{\mathrm{B}} T}
$$

We obtain $n_{\text {id }}=1$ by comparing Equation 4 with Equation 10, and then $\delta=\Delta=2$.

For the recombination between free electrons (holes) and trapped holes (electrons), the density of trapped electrons $n_{\mathrm{t}}$ (holes $p_{\mathrm{t}}$ ) is given by $n_{\mathrm{t}}=p_{\mathrm{t}} \propto \exp \left(q V / 2 E_{\mathrm{ch}}\right)$ assuming the same exponential tail $E_{\mathrm{ch}}$ due to an energetic disorder. Thus, $R$ is described as

$$
R \propto n_{\mathrm{f}} p_{\mathrm{t}} \propto n_{\mathrm{t}} p_{\mathrm{f}} \propto \exp \left\{\frac{q V}{k_{\mathrm{B}} T}\left(\frac{1}{2}+\frac{k_{\mathrm{B}} T}{2 E_{\mathrm{ch}}}\right)\right\}
$$


Therefore, $n_{\text {id }}$ and $m$ are expressed as

$$
\begin{gathered}
n_{\mathrm{id}}=\left(\frac{1}{2}+\frac{k_{\mathrm{B}} T}{2 E_{\mathrm{ch}}}\right)^{-1}, \\
m=\frac{2 E_{\mathrm{ch}}}{k_{\mathrm{B}} T},
\end{gathered}
$$

Finally, $\delta$ and $\Delta$ are expressed as

$$
\begin{gathered}
\delta=\frac{E_{\text {ch }}}{k_{\mathrm{B}} T}+1 . \\
\Delta=\frac{k_{\mathrm{B}} T}{E_{\text {ch }}}+1 .
\end{gathered}
$$

Note that the exponential tail $E_{\mathrm{ch}}$ for the highest occupied molecular orbital (HOMO) and the lowest unoccupied molecular orbital (LUMO) are not necessarily identical. Equations 14 and 15 mean that $\delta$ becomes larger than 2 while $\Delta$ becomes less than 2 with increasing $E_{\mathrm{ch}}$ over $k_{\mathrm{B}} T$. In summary, $\delta$ and $\Delta$ would be different for trap-assisted recombination in the presence of an energetic disorder.

\section{Langevin Recombination}

Recombination of free charge carriers in noncrystalline semiconductors is often described by the Langevin theory. ${ }^{60}$ When the mobility of localised charge carriers is relatively low, the mean free path of the carriers is less than the radius of capture of one carrier by the other. ${ }^{61}$ In this case, the recombination rate is proportional to the probability of opposite charges finding each other. This is the case for polymer semiconductors. As polymer semiconductors are disordered materials, the recombination rate is limited by localised hopping transport with a low mobility as opposed to crystalline semiconductors with a high mobility where delocalised charge transport is dominant. ${ }^{61}$

In the Langevin framework, the Langevin recombination rate coefficient $\gamma_{L}$ can be derived by solving the Debye-Smoluchowski equation with an assumption of the validity of the Einstein relationship $D=\mu k_{\mathrm{B}} T / q$, where $D$ is the relative diffusion coefficient $\left(D=D_{\mathrm{n}}+D_{\mathrm{p}}\right) .62,63$

$$
\gamma_{\mathrm{L}}=4 \pi D r_{\mathrm{c}}=\frac{q \mu}{\varepsilon_{0} \varepsilon_{\mathrm{r}}}
$$

Equation 16 tells us that the Langevin recombination rate is proportional to the mobility. This means that if you obtain a higher mobility polymer material to shorten the charge collection time, you would also experience a faster NGR, resulting in a negligible or often a negative impact on charge collection efficiency. In other words, a high FF could not be obtained with a thick active layer if the recombination is governed by the Langevin framework.

\section{Reduced NGR}

In order to obtain a long charge carrier lifetime and a high FF with a thick active layer beyond the limitation of the Langevin theory, "reduced Langevin" description is of particular importance. In this context, the recombination rate is "reduced" as

$$
\gamma=\zeta \gamma_{\mathrm{L}}
$$

where $\zeta$ is often called the Langevin reduction factor. Indeed, some blend systems have shown reduced NGR rates, which are 2-4 orders of magnitude lower than the Langevin recombination rate, i.e. $\zeta$ is on the order of $10^{-2}-10^{-4}$, as will be summarised in Table 1 . In such cases, high FFs can be obtained even with thicker active layers. It is, therefore, important to understand what is the origin of the reduced Langevin recombination and what controls the reduction factor. Proposed theories for the reduced NGR will be described later.

\section{NGR Measurement Techniques}

We now turn to recent experimental findings regarding NGR. Dynamics of NGR has been measured by various techniques. Here, we briefly summarise some major techniques before moving to detailed discussion.

\section{Light Intensity Dependence of $J_{\text {sc }}$}

A classical method to discuss NGR is light intensity dependent $J-V$ measurements where $J_{S C}$ is plotted against light intensity $1 .{ }^{64-68}$ Then the $J_{\text {SC }}$ is fitted by a power-law function $J_{\text {SC }} \propto I^{\alpha}$ (Figure 3 ). Note that this $\alpha$ is different from that in Equations 22-26, and 67. If the NGR loss can be disregard under short-circuit condition, $\alpha$ would be close to unity. On the other hand, $\alpha$ would be close to $1 / 2$ when the NGR loss is significant. The fraction of charge carriers that recombined bimolecularly is given as $\alpha^{-1-1}$. This method provides useful information with a simple measurement setup, however, charge dynamics in the active layer remains unclear. Note that this method can predict the loss fraction of charge carriers correctly when NGR is the major intensity dependent loss mechanism and space-charge effects are not operative.

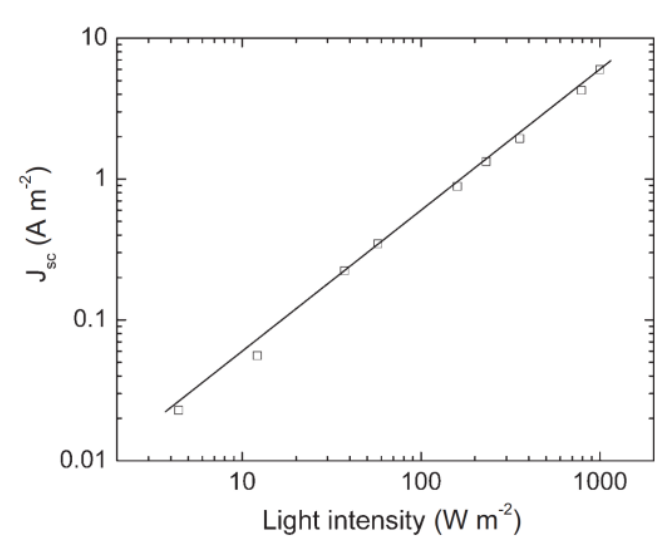

Figure 3. Log-log plots of short-circuit current density $\left(J_{\mathrm{SC}}\right)$ against light intensity of an MDMO-PPV/PCNEPV solar cell. The solid line represents a linear relation with a slope of unity. Reprinted with permission from ref. 64. Copyright 2007 WILEY-VCH Verlag Gmbh \& Co. KGaA, Weinheim.

\section{Transient Photovoltage/Photocurrent (TPV/TPC) Measurement}

Transient photovoltage/photocurrent (TPV/TPC) measurements (Figure $4 a, b$ ) are optoelectronic techniques with which we evaluate 
(a)

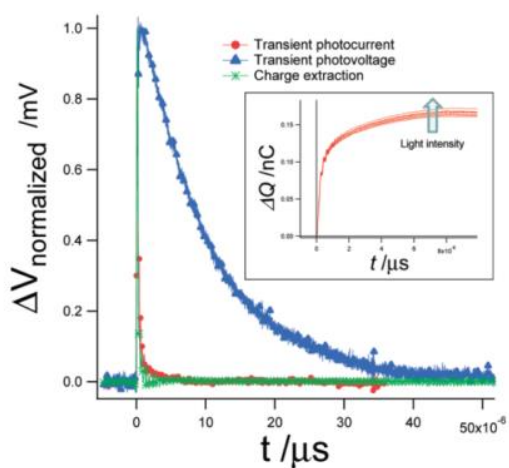

(b)

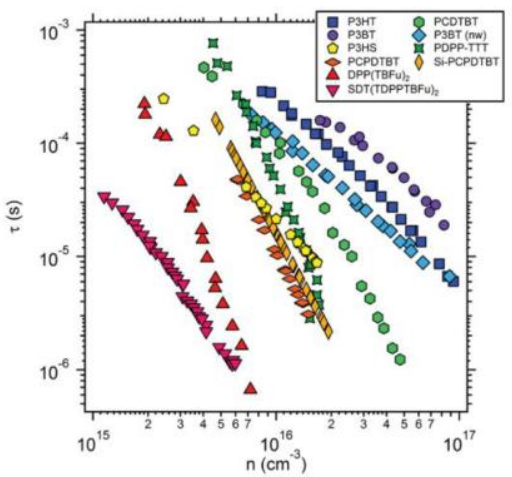

(c)

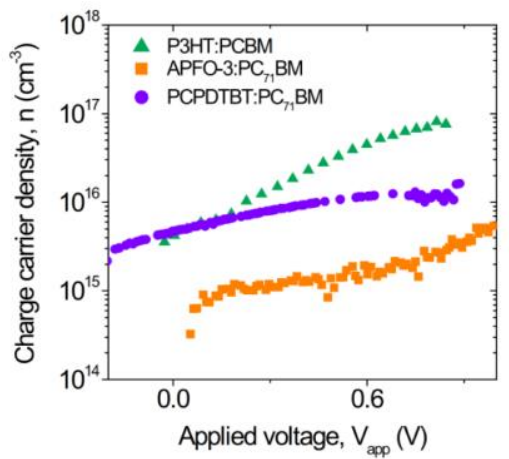

Figure 4. (a) Normalised TPV, TPC, and CE transients acquired for a $\mathrm{P} 3 \mathrm{HS} / \mathrm{PC}_{61} \mathrm{BM}$ solar cell under $\sim 1$ sun steady-state background illumination condition. The inset shows the charging transients obtained by integration of the TPC transients under various background light intensities from dark to 1 sun, indicating the amount of additional charges $\Delta q$ is independent of the background light intensity. Reprinted with permission from ref. 74. Copyright 2011 American Chemical Society. (b) Log-log plots of charge carrier lifetime against charge carrier density for solar cells based on various blend systems. Reprinted with permission from ref. 77. Copyright 2012 American Chemical Society. (c) Semilogarithmic plots of the steady-state charge carrier densities against applied bias. Reprinted with permission from ref. 81. Copyright 2013 American Chemical Society.

charge carrier lifetime $\tau_{n}$ as a function of charge carrier density $n .{ }^{69}$ 79 Full details of these techniques are described in ref. 74. The advantage of these techniques is that we can obtain these recombination parameters under steady-state device operating condition. In the TPV measurements, a solar cell is held at open circuit with $\sim 1 \mathrm{M} \Omega$ input impedance. The background white light is illuminated under various intensities to produce free charge carriers in the active layer. Under open-circuit condition, charges cannot leave the device instead they accumulate and provide a voltage $V_{\mathrm{Oc}}$. Then, a weak pulse laser is provided to give a small amount of extra charges, resulting in a small perturbation for the $V_{\text {oc. }}$. Additional charges recombine with a time constant of $\tau_{\Delta n}$, where pseudo firstorder reaction is assumed, and finally the voltage decays back to $V_{O C}$ is evaluated. As described in ref. 74 , the time constant $\tau_{\Delta n}$ is related to charge carrier lifetime $\tau_{n}$ as $\tau_{n}=\delta \tau_{\Delta n}$. On the other hand, in TPC measurements, the device is held at a quasi short circuit with a small (typically $\sim 50 \Omega$ ) resistor inserted in parallel to the device. The voltage response of the device after the pump laser under various background illumination is also measured in the TPC measurements. The TPC transients are converted into current transients based on the Ohm's law and integrated over the time to estimate the amount of additional charges $\Delta q$. Then, the differential capacitance $\mathrm{d} C$ is calculated as $\mathrm{d} C\left(V_{\text {OC }}\right)=\Delta q / \Delta V_{0}\left(V_{\text {OC }}\right)$ where $\Delta V_{0}\left(V_{\text {OC }}\right)$ is the amplitude of the TPV transients under the same background illumination. The total carrier density at open circuit $n\left(V_{\text {OC }}\right)$ is evaluated by integrating $\mathrm{d} C$ from $0 \mathrm{~V}$ to $V_{\text {oc. }}{ }^{74}$ As shown in Equations 18 and $19, \tau_{\Delta n}$ and $n\left(V_{o C}\right)$ exponentially decrease and increase with increasing $V_{O c}$, respectively. The exponent $\lambda$ in Equation 20 is equal to $m / v$.

$$
\begin{gathered}
\tau_{\Delta n}=\tau_{\Delta n_{0}} \exp \left(-\frac{q V_{\mathrm{OC}}}{v k_{\mathrm{B}} T}\right) \\
n\left(V_{\mathrm{OC}}\right)=n_{0} \exp \left(\frac{q V_{\mathrm{OC}}}{m k_{\mathrm{B}} T}\right) \\
\tau_{n}=\tau_{0} n^{-\lambda}=\delta \tau_{\Delta n}=(1+\lambda) \tau_{\Delta n}
\end{gathered}
$$

$$
\lambda=\frac{m}{v}
$$

Note that the effect of current leakage due to small shunt resistance should be considered especially for measurement region of low light intensity. ${ }^{74}$ To eliminate the effect, you should choose devices with enough shunt resistance and restrict the measurement within light intensity region where the linear relationship between the logarithm of light intensity and open-circuit voltage.

\section{Charge Extraction (CE) Measurement}

Charge extraction (CE) measurements (Figure 4a,c) are also optoelectronic techniques with which we can evaluate $n$ under various applied voltages. ${ }^{71,72,74,76,80-82}$ In the CE measurements, a solar cell is illuminated with white light under various intensities and held at a certain voltage $V_{\text {app }}$. Once the device reaches a steady-state, the white light is turned off and simultaneously the device is shorted using a small $(\sim 50 \Omega)$ resistor. The device is discharged and produces a voltage signal that is measured across the small resistor. By converting the voltage decay to current decay based on the Ohm's law and integrating the current over time, $n$ is evaluated directly. The assumption in these measurements is that all charges in the device are extracted under short-circuit condition without any loss such as NGR. However, this is not the case in some blend systems. To overcome this problem, bias-assisted charge extraction (BACE) measurements are employed. In these measurements, a reverse bias is applied to the device instead of under short circuit to enhance charge extraction.

Time-resolved charge extraction (TRCE) measurements also revealed an extraction transient, but it tracks the time evolution of charge density. ${ }^{83-85} \mathrm{~A}$ solar cell is illuminated with a laser pulse instead of white light, under open-circuit condition. By short-circuiting the device after a certain time delay, charge carrier density at each delay 
time is obtained. From the gradient of the charge carrier decay, the NGR rate constant is evaluated.

\section{Transient Absorption Spectroscopy (TAS)}

Transient absorption spectroscopy (TAS) (Figure 5) is one of the most powerful techniques for observing transient species such as charge carriers generated after photoexcitation. ${ }^{47,86-93}$ In the TA measurement, the probe light intensity transmitted through a sample is detected with and without the photoexcitation $\left(I\right.$ and $I_{0}$, respectively). From the intensity ratio, the absorbance change is given by $\triangle O D=\log \left(I_{0} / I\right)$. Note that, in many cases, TA measurements are also presented as the fractional differential transmission $\Delta T / T$. For fractional changes on the order of a few percent or smaller, it can be shown that $\Delta T / T \approx-(\ln 10) \Delta O D$. Consequently, photoproducts provide positive signals in the $\triangle O D$ but emission or ground-state bleaching results in negative signals in the $\triangle O D$. According to the Beer-Lambert law, the $\triangle O D$ is proportional to the density of transient species $\Delta \mathrm{OD}=n \sigma l$ where $n, \sigma$, and $/$ are the density of transients, absorption cross-section, and optical pass length, respectively. The NGR rate constant is evaluated from the time evolution of charge carrier density $n(t)$. The $n(t)$ for NGR can be fitted with an empirical power-law equation.

$$
n(t)=\frac{n(0)}{(1+a t)^{\alpha}}
$$

where $n(0)$ is the initial charge density and $a$ and $\alpha$ are kinetic parameters. Note that this $\alpha$ is different from the previous one and that in Equation 67. On the basis of Equation 1, time-dependent NGR rate constant $\gamma(t)$ is given by

$$
\gamma(t)=-\frac{1}{n^{2}(t)} \frac{\mathrm{d} n(t)}{\mathrm{d} t}=\frac{a \alpha}{n(0)}(1+a t)^{\alpha-1}
$$

On the other hand, charge density-dependent NGR rate constant $\gamma(n)$ is given by

$$
\gamma(n)=\frac{a \alpha}{n}\left(\frac{n}{n(0)}\right)^{\frac{1}{\alpha}}
$$

Thus, $\tau_{n}$, which is defined as reciprocal of first-order rate constant, is given by ${ }^{74}$

$$
\tau_{n}=\frac{1}{\gamma(n) n}=\frac{n(0)^{\frac{1}{\alpha}}}{a \alpha} n^{-\frac{1}{\alpha}}
$$

Comparing Equations 18-21 from TPV/TPC with Equation 25 from TAS, the following relations are obtained.

$$
\begin{gathered}
\alpha=\frac{1}{\lambda} \\
a=\frac{\lambda n(0)^{\lambda}}{(\lambda+1) \tau_{\Delta n_{0}} n_{0}^{\lambda}}
\end{gathered}
$$

Therefore, the empirical equation can be expressed with the kinetic parameters obtained from TPV/TPC measurements as follows:

$$
n(t)=n(0)\left[1+\frac{\lambda n(0)^{\lambda}}{(\lambda+1) \tau_{\Delta n_{0}} n_{0}^{\lambda}} t\right]^{-\frac{1}{\lambda}}
$$

(a)

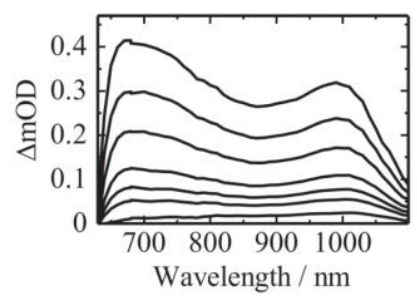

(b)

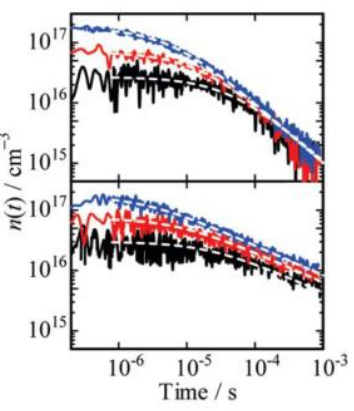

Figure 5. (a) TA spectra of RR-P3HT/PC 61 BM blend films measured at $0.5,1,2,5$, and $10 \mu$ s from top to bottom excited at $400 \mathrm{~nm}$. Reprinted with permission from ref. 93. Copyright 2016 IEEE. (b) Charge carrier density decays of RR$\mathrm{P} 3 \mathrm{HT}: \mathrm{PC}_{61} \mathrm{BM}$ blend films measured at $700 \mathrm{~nm}$ (upper panel) and $1000 \mathrm{~nm}$ (lower panel) excited at $0.8,1.8$, and $4.7 \mu \mathrm{J} \mathrm{cm}^{-2}$ from bottom to top in each panel. The broken lines represent fitting curves with Equation 22. Reprinted with permission from ref. 87. Copyright 2010 American Chemical Society.

Note that TAS is a transient measurement where charge carriers are not always under steady-state condition as is the case in operating devices. This is also the case with CELIV and TDCF as will be described later.

\section{Photoinduced Charge Carrier Extraction by Linearly Increasing Voltage (Photo-CELIV) Technique}

Photoinduced charge carrier extraction by linearly increasing voltage (photo-CELIV) technique (Figure 6) is used to simultaneously evaluate the charge carrier mobility and NGR rate in a solar cell. ${ }^{94-105}$ First, a laser pulse is applied to a solar cell while applying a constant voltage to minimise charge extraction. After a certain delay time $t_{\mathrm{d}}$ the remaining charges are extracted by a linearly increasing voltage. The resulting transient current exhibits an initial step $j_{0}$ arising from

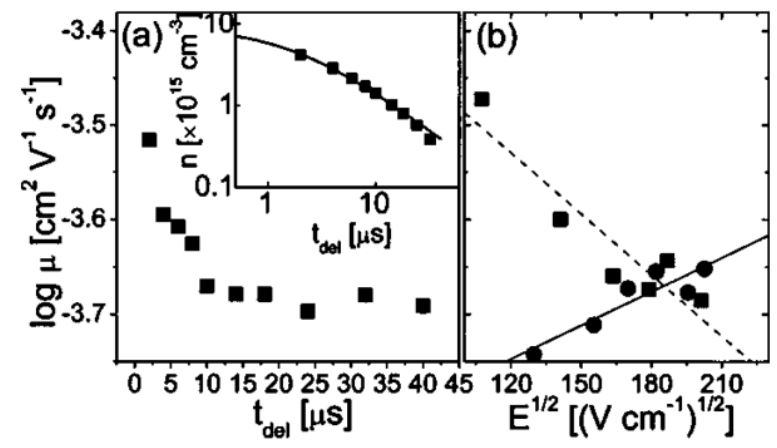

Figure 6. The logarithm of charge carrier mobility plotted against the delay time (a) and the square root of electric field

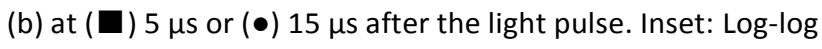
plots of charge carrier density against delay time. The solid line represents a fitting curve. Reprinted with permission from ref. 97. Copyright 2005 American Institute of Physics. 
geometrical capacitance of the device and reaches the maximum current $j_{0}+\Delta j$ at a certain time $t_{\max }$. Using $j_{0}, \Delta j$, and $t_{\max }$, the charge carrier mobility is calculated as

$$
\mu=\frac{2 d^{2}}{3 A t_{\max }^{2}\left(1+0.36 \frac{\Delta j}{j_{0}}\right)}
$$

where $A$ is the voltage rise speed. Charge carrier density is also evaluated from the difference between collected charges with and without a laser pulse. The time evolution of charge carrier density is obtained by varying $t_{\mathrm{d}}$, from which the NGR rate constant is evaluated.

\section{Time-Delayed Collection Field Measurement}

Time-delayed collection field (TDCF) (Figure 7) is a technique that can be used to evaluate charge recombination loss including fielddependent GR and NGR. ${ }^{106-109} \mathrm{~A}$ laser pulse is applied to a solar cell under a pre-bias of $V_{\text {pre. }}$. After a certain delay time $t_{\mathrm{d}}$, a voltage for charge collection $V_{\text {col }}$ is applied to the device to extract all the remaining charges in the active layer. The voltage signal is recorded through a small resistor and transferred to the current. By integrating the current, the amount of extracted charges by $V_{\text {pre }}\left(Q_{\text {pre }}\right)$ and that by $V_{\text {col }}\left(Q_{\text {col }}\right)$ are estimated. Total extracted charges $Q_{\text {total }}\left(=Q_{\text {pre }}+\right.$ $\left.Q_{\text {col }}\right)$ as a function of $V_{\text {pre }}$ enables us to evaluate field-dependence of GR losses. On the other hand, $Q_{\text {col }}$ as a function of $t_{\mathrm{d}}$ under a condition of constant $V_{\text {pre }}$ provides the time dependence of collected charges. The NGR rate constant is calculated by analysing the charge decay.

\section{Steady-State J-V Measurement}

Wetzelaer et al. proposed a way for evaluating $\zeta$ by steady-state $J-V$ measurements (Figure 8). ${ }^{110}$ In this method, three types of devices

(a)

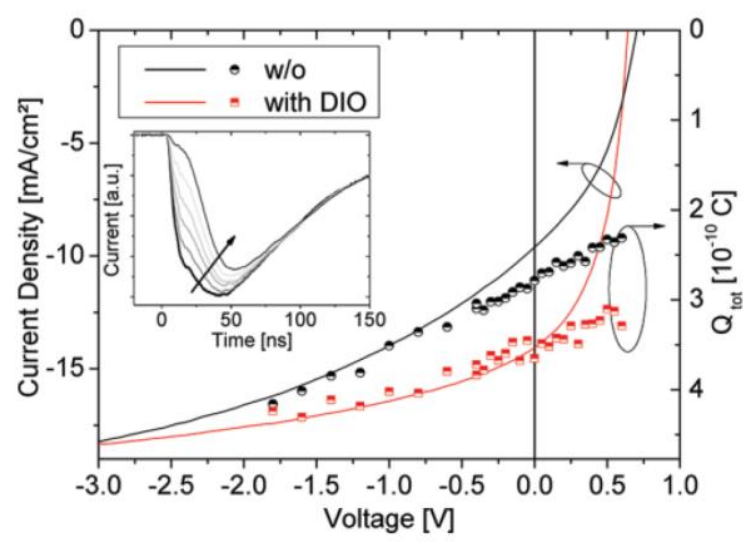

are fabricated: hole-only devices, electron-only devices, and doublecarrier devices. The former two single-carrier devices are used for space-charge limited current (SCLC) measurements. The current follows the Mott-Gurney law:

$$
J_{\mathrm{h}(\mathrm{e})}=\frac{9}{8} \varepsilon_{0} \varepsilon_{\mathrm{r}} \mu_{\mathrm{h}(\mathrm{e})} \frac{V^{2}}{d^{3}}
$$

where $J_{\text {h(e) }}$ represents the current in hole (or electron)-only devices. For double carrier devices, the active layer is sandwiched by an electron-injecting electrode such as LiF/Al and a hole-injecting electrode such as Au/PEDOT:PSS. They assumed the current in double-carrier devices $J_{D}$ as

$$
J_{\mathrm{D}}=\sqrt{J_{\mathrm{BB}}^{2}+J_{\mathrm{IP}}^{2}}
$$

where $J_{\mathrm{BB}}$ and $J_{\mathrm{IP}}$ are the current under two extreme conditions called back to back and injected plasma, respectively. ${ }^{111}$

$$
\begin{gathered}
J_{\mathrm{BB}}=\frac{9}{8} \varepsilon_{0} \varepsilon_{\mathrm{r}}\left(\mu_{\mathrm{h}}+\mu_{\mathrm{e}}\right) \frac{V^{2}}{d^{3}} \\
J_{\mathrm{IP}}=\left(\frac{\mathbf{9}}{\mathbf{8}} \boldsymbol{\pi}\right)^{\frac{1}{2}} \varepsilon_{\mathbf{0}} \varepsilon_{\mathbf{r}}\left(\frac{\mathbf{2} \mu_{\mathrm{h}} \boldsymbol{\mu}_{\mathrm{e}}}{\zeta}\right)^{\frac{1}{2}} \frac{V^{2}}{\boldsymbol{d}^{3}}
\end{gathered}
$$

Using the above equations, $\zeta$ is expressed as

$$
\zeta=\frac{16 \pi}{9} \frac{\mu_{\mathrm{h}} \mu_{\mathrm{e}}}{\mu_{\mathrm{eff}}^{2}-\left(\mu_{\mathrm{h}}+\mu_{\mathrm{e}}\right)^{2}}
$$

where $\mu_{\text {eff }}$ is the effective mobility in double-carrier devices.

$$
J_{\mathrm{D}}=\frac{9}{8} \varepsilon_{0} \varepsilon_{\mathrm{r}} \mu_{\mathrm{eff}} \frac{V^{2}}{d^{3}}
$$

This is a brief technique because you do not need any measurement setups other than conventional $J-V$ measurement equipment. Note

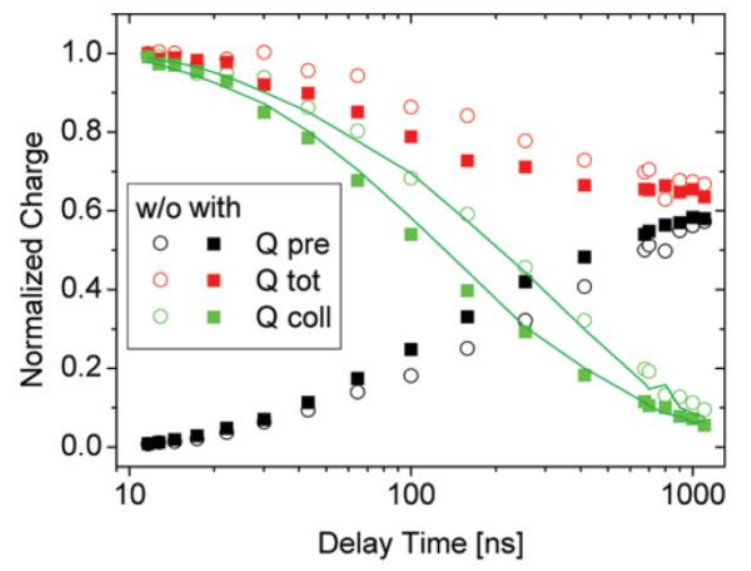

Figure 7. (a) Left scale: photocurrent density for blends with (red) and without (black) processing additive diiodooctane (DIO). Right scale: total extracted charges for blends with (red) and without (black) DIO plotted against prebias voltage during excitation. The inset shows the corresponding TDCF transients for blends with DIO and $V_{\text {pre }}$ ranging from -0.4 to $0.6 \mathrm{~V}$. (b) $Q_{\text {pre }}\left(t_{\mathrm{d}}\right), Q_{\text {coll }}\left(t_{\mathrm{d}}\right)$, and $Q_{\text {tot }}\left(t_{\mathrm{d}}\right)$ normalised to the initially generated charge under a prebias voltage of $0.3 \mathrm{~V}$ plotted against delay time for blends with (filled symbols) and without (open symbols) DIO. The solid lines represent NGR fitting curves. Reprinted with permission from ref. 107. Copyright 2012 American Chemical Society. 


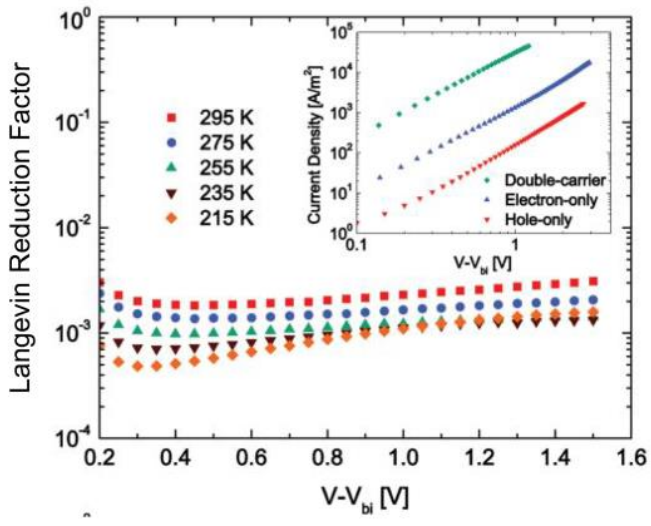

Figure 8. Semilogarithmic plots of temperature-dependent Langevin reduction factor calculated by Equation 34 for an RR-P3HT/PC 61 BM solar cell. The inset shows the $J-V$ curves of the three devices measured at room temperature. Reprinted with permission from ref. 110. Copyright 2013 WILEY-VCH Verlag GmbH \& Co. KGaA, Weinheim.

that the measurement does not track actual recombination dynamics like in other transient measurements.

\section{Consistency among Different Techniques}

The question is whether there is a consistency among different techniques, and whether we should select an appropriate measurement technique depending on the situation. A major difference among the techniques is whether the measured sample is a solar cell device or a film on substrate without electrodes. It seems appropriate to use a device structure to simulate the operating condition whereas electrodes may induce undesirable effects of shunts or surface recombination which prevents direct observation of bulk NGR loss.

Shuttle et al. employed reflection-mode TAS on a blend film with and without electrodes and TPV/TPC measurement on a solar cell to compare these results. ${ }^{69}$ An excellent agreement was obtained among the time evolutions of charge carrier density, indicating that TAS and TPV/TPC methods provide the same information about NGR loss. Clarke et al. conducted five different measurements for the same blend system. ${ }^{112}$ The measurements are divided into three categories: optical (TAS), optoelectronic (TPV, photo-CELIV, and TRCE), and electrical (electrochemical impedance spectroscopy: EIS) Figure 9 shows the $\tau_{n}$ plotted against $n$ measured by five techniques. The dashed lines show that at a high charge density, the power law fits the equation $\tau_{n}=\tau_{0} n^{-\lambda}$. The $\tau_{n}$ evaluated at high charge carrier densities $\left(>3 \times 10^{16} \mathrm{~cm}^{-3}\right.$ ) showed similar values in all methods, except for EIS. They attributed the deviation from other techniques observed in EIS to the problem of unsophisticated analysis method. The exponent $\lambda$ changed with the techniques. Photo-CELIV exhibited the smallest $\lambda$ of 1.4 , which was likely due to charge leakage or a short integration time. On the other hand, $\lambda$ of 2.1-2.6 was obtained for TPV, TRCE, and TAS. In addition, higher $\lambda$ at a low charge density was observed for TPV and TRCE. They mentioned the possible mechanism of surface recombination or inhomogeneous charge

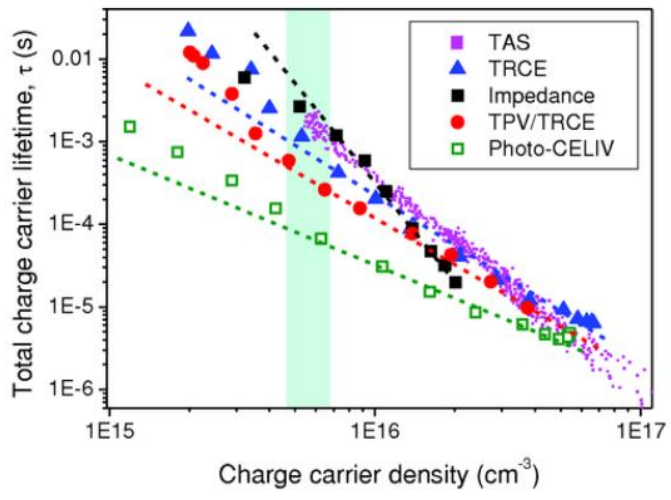

Figure 9. Log-log plots of charge carrier lifetime against charge carrier density for the five techniques. The dashed lines represent fitting lines at high charge density with a power-law equation $\tau=\tau_{0} n^{-\lambda}$. The shaded region represents the range where each of the techniques deviates from this equation (except TAS). Reprinted with permission from ref. 112. Copyright 2015 WILEY-VCH Verlag Gmbh \& Co. KGaA, Weinheim.

distribution. They proposed that a combination of TAS and TRCE is appropriate for investigation of the NGR dynamics over a wide charge density range.

All these studies suggest that there is a consistency among different techniques when we evaluate the NGR rate under 1 sun illumination condition. However, when we evaluate the NGR rate over a wide charge density range or determine the NGR reaction order, it would be necessary to carefully consider the effects of current leakage or surface recombination, especially for techniques employing a device structure.

\section{Reduced NGR}

The reduction factors $\zeta$ reported for various blends are summarised in Table 1. Some trends can be seen in the data depending on the type of donor or acceptor material, crystallinity of polymers, or device fabrication condition. In polymer/fullerene systems, blends employing highly crystalline polymers such as regioregular (RR)-P3HT, DT-PDPP2T-TT, and PNTZ4T exhibit a small reduction factor of 0.01 or less (the chemical structure of materials are shown in the Appendix). Blends employing polymers with low crystallinity such as PCPDTBT and PTB7 exhibit moderate reduction factor of about 0.1. Amorphous polymers such as MDMO-PPV and regiorandom (RRa)P3HT exhibit a reduction factor of 1 , indicating Langevin-type diffusion-limited recombination. Note that morphological change by introducing additives can decrease the reduction factor in some blends. On the other hand, polymer/nonfullerene acceptor (NFA) systems, a wide range of values are reported. For polymer/small molecule NFA (SMNFA) systems, reduction factors between 0.01-1 are reported. Recent studies have shown relatively small reduction factors less than 0.05 for blend films employing more crystalline polymers such as PTB7-Th and PffBT4T-2OD. Blends employing fluorinated SMNFA such as ITIC-2F and IT-4F exhibit reduction factors 
Table 1. Experimental reduction factors in each blend system.

\begin{tabular}{|c|c|c|c|c|c|c|}
\hline Group & Donor & Acceptor & Remark & Reduction factor & Measurement & Ref. \\
\hline \multirow[t]{28}{*}{ polymer/fullerene } & RR-P3HT & $\mathrm{PC}_{61} \mathrm{BM}$ & pristine & $0.1-1$ & CELIV, TPV & 113,114 \\
\hline & & & annealed & $0.0001-0.01$ & TAS, CELIV, TPV & $86,113-115$ \\
\hline & RR-P3HT & $\mathrm{PC}_{71} \mathrm{BM}$ & & 0.006 & TDCF & 106 \\
\hline & DT-PDPP2T-TT & $\mathrm{PC}_{71} \mathrm{BM}$ & & 0.0042 & TPV & 115 \\
\hline & PSBTBT & $\mathrm{PC}_{71} \mathrm{BM}$ & & 0.04 & TPV & 116 \\
\hline & PNTz4T & $\mathrm{PC}_{71} \mathrm{BM}$ & & 0.01 & TPV & 117 \\
\hline & PNTZ4TF2 & $\mathrm{PC}_{71} \mathrm{BM}$ & & 0.03 & TPV & 117 \\
\hline & PNTz4TF4 & $\mathrm{PC}_{71} \mathrm{BM}$ & & 0.1 & TPV & 117 \\
\hline & РВTTT & $\mathrm{PC}_{61} \mathrm{BM}$ & $1: 1$ & $\sim 0.3$ & CELIV & 118 \\
\hline & & & $1: 4$ & 0.03 & CELIV & 118 \\
\hline & NT812 & $\mathrm{PC}_{71} \mathrm{BM}$ & & 0.00125 & $J-V^{a}$ & 119 \\
\hline & PPDT2FBT & $\mathrm{PC}_{71} \mathrm{BM}$ & & 0.01 & IPDA $^{b}$ & 120 \\
\hline & PIPCP & $\mathrm{PC}_{61} \mathrm{BM}$ & & 0.2 & IPDA & 120 \\
\hline & PDPP-TNT & $\mathrm{PC}_{71} \mathrm{BM}$ & & 1 & CELIV & 121 \\
\hline & PffBT4T-2OD & $\mathrm{PC}_{71} \mathrm{BM}$ & & 0.1 & TPV & 122 \\
\hline & PCPDTBT & $\mathrm{PC}_{71} \mathrm{BM}$ & $w / o$ & 0.2 & TDCF & 123 \\
\hline & & & DIO 3\% & 0.07 & TDCF & 123 \\
\hline & F-PCPDTBT & $\mathrm{PC}_{71} \mathrm{BM}$ & w/o & 0.14 & TDCF & 123 \\
\hline & & & DIO 1\% & 0.04 & TDCF & 123 \\
\hline & & & DIO 3\% & 0.03 & TDCF & 123 \\
\hline & PTB7 & $\mathrm{PC}_{71} \mathrm{BM}$ & w/o & 0.1 & IPDA & 120 \\
\hline & & & DIO 3\% & 0.03 & IPDA & 120 \\
\hline & PTB7-Th & $\mathrm{PC}_{71} \mathrm{BM}$ & w/o & 0.1 & IPDA & 120 \\
\hline & & & DPE 3\% & 0.03 & IPDA & 120 \\
\hline & PCDTBT & $\mathrm{PC}_{61} \mathrm{BM}$ & & $0.3-1$ & TOF $^{c}$ & 124 \\
\hline & RRa-P3HT & $\mathrm{PC}_{61} \mathrm{BM}$ & & 1 & CELIV & 125 \\
\hline & MDMO-PPV & $\mathrm{PC}_{61} \mathrm{BM}$ & & 1 & CELIV & 98 \\
\hline & PTQ1 & $\mathrm{PC}_{71} \mathrm{BM}$ & & 0.05 & CELIV, TRMC $^{d}$ & 32 \\
\hline \multirow[t]{12}{*}{ polymer/SMNFA } & PBDB-T & ITIC & & 0.5 & $J-V$ & 126 \\
\hline & PBDBT-2F & ITIC & & 0.283 & $J-V$ & 127 \\
\hline & & IT-4F & & 0.0371 & $J-V$ & 127 \\
\hline & NT812 & ITIC & & 0.2 & BACE & 128 \\
\hline & PTB7-Th & ITIC-2F & & 0.013 & $J-V$ & 129 \\
\hline & РMOT40 & IDIC & & $0.001-0.01$ & TPV & 130 \\
\hline & PBDB-T & IDTIC & & 1.22 & CELIV & 131 \\
\hline & & IDTTIC & & 0.16 & CELIV & 131 \\
\hline & PBDB-T & ITCC & & 0.68 & $J-V$ & 132 \\
\hline & PTB7-Th & IEICO-4F & & 0.027 & $J-V$ & 132 \\
\hline & РffBT4T-2OD & FBR & & 0.05 & TPV & 122 \\
\hline & PTB7-Th & bay-di-PDI & & 0.1 & TAS & 51 \\
\hline \multirow[t]{5}{*}{ polymer/polymer } & RR-P3HT & N2200 & $\begin{array}{c}200{ }^{\circ} \mathrm{C} \\
\text { dried }\end{array}$ & 0.002 & TDCF & 133 \\
\hline & & & RT dried & 0.015 & TDCF & 133 \\
\hline & PBDB-T & N2200 & & 0.43 & $J-V$ & 134 \\
\hline & PTzBI & N2200 & & 0.28 & $J-V$ & 134 \\
\hline & PTQ1 & N2200 & & $0.1^{e}$ & TAS & 135 \\
\hline
\end{tabular}

${ }^{a}$ Steady-state $J-V$ measurements described in the main text. ${ }^{b}$ Impedance-photocurrent device analysis (IPDA) demonstrated by Heiber et al. Note that reduction factors are evaluated at maximum power point. ${ }^{c}$ Time-of-flight. ${ }^{d}$ Time-resolved microwave conductance. ${ }^{e}$ Reduction factor when using mobilities determined from SCLC measurement. Details are given in ref. 135 
less than 0.04. For polymer/polymer systems, although it is difficult to find trends owing to the lack of experimental reports, relatively small reduction factors were reported for blend films employing RR$\mathrm{P} 3 \mathrm{HT}$. In this section, we will discuss suggestive results among these systems.

\section{Crystalline Polymer-Based Solar Cells}

Many researchers have pointed out the importance of crystallinity for suppressing NGR. Hamilton et al. studied the annealing effects on NGR in OPVs based on RR-P3HT/PC ${ }_{61} B M$ blends. ${ }^{114}$ It is well known that RR-P3HT/PC ${ }_{61} \mathrm{BM}$ devices exhibit relatively high $\mathrm{FF}(>0.65)$ even with a thick (>200 nm) active layer. ${ }^{3}$ RR-P3HT, a well-known benchmark polymer used for OPVs, forms a $\pi$-stacking crystalline structure in thin films, in which the crystallinity of RR-P3HT increases after thermal annealing. They performed TPV/TPC measurements for pristine and annealed RR-P3HT/PC ${ }_{61} B M$ devices, and found that the charge carrier lifetime $\tau_{n}$ of the annealed device is one order of magnitude longer than that of the pristine device. As shown in Figure 10 , the reduction factor for the annealed device $\zeta$ was significantly reduced to $10^{-3}-10^{-2}$ as compared to $10^{-1}$ for the pristine device. Such significant suppression of NGR was also observed in OPVs consisting of crystalline low-bandgap polymers. Fukuhara et al. have studied the NGR dynamics in OPVs based on blends of DT-PDPP2TTT/PC $C_{71} \mathrm{BM}^{115}$ DT-PDPP2T-TT is also a crystalline polymer and shows a considerably high $J_{\mathrm{SC}}$ of $20.07 \mathrm{~mA} \mathrm{~cm}^{-2}$ and a high $\mathrm{FF}$ of 0.70 with an optimum thickness of $340 \mathrm{~nm}$ when blended with $\mathrm{PC}_{71} \mathrm{BM} .{ }^{136}$ The reduction factor was estimated through TPV/TPC measurements to be on the order of $10^{-3}$, indicating that NGR is substantially suppressed in these systems. These results shed light on the importance of crystallinity for suppressing NGR and boosting FF. Increasing crystallinity is, of course, not a sufficient condition to achieve a high FF with thick active layers. Fukuhara et al. also investigated the NGR dynamics in PSBTBT/PC 71 BM devices. ${ }^{116}$ PSBTBT is also a crystalline polymer although the crystalline size is

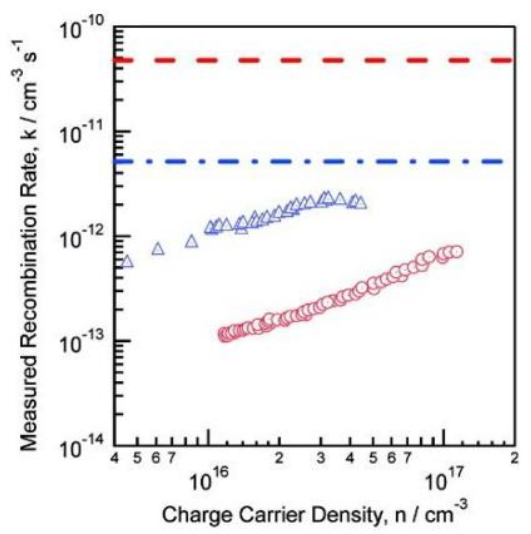

Figure 10. Log-log plots of recombination rate constant against charge carrier density for annealed (red circles) and pristine (blue triangles) solar cells in comparison with the Langevin recombination rate constant calculated using hole mobility of annealed (red dashed line) and pristine (blue dash-dot line) blend films. Reprinted with permission from ref. 114. Copyright 2010 American Chemical Society. smaller than that of annealed RR-P3HT. ${ }^{48}$ However, the optimum thickness is as thin as $\sim 100 \mathrm{~nm}$, and otherwise FF is degraded with the increase in thickness. They found by TPV/TPC measurements that $\zeta$ is on the order of $10^{-2}$, indicating that NGR is sufficiently suppressed in this system. Nonetheless, FF of this device was as low as 0.46 with an active layer thickness of $180 \mathrm{~nm}$. Using recombination parameters obtained by TPV/TPC measurements, they tried to reproduce the observed $J-V$ curve. However, the estimated $J-V$ curve largely overestimated the photocurrent over a whole voltage range from short circuit to open circuit. The deviation from the measured data became larger with increasing applied bias. This behaviour might be a sign of electric field dependent charge generation. However, TA measurements performed under various applied voltages showed that the charge generation yield hardly depends on the applied voltage from 0 to $1 \mathrm{~V}$. Rather, they speculated that the charge density at short circuit would be much higher than they assumed. The hole mobility of PSBTBT is as low as a quarter of the electron mobility of $\mathrm{PC}_{71} \mathrm{BM}$. Such an imbalanced mobility would cause charge accumulation in the active layer, which screens the built-in electric field. They concluded that the low FF for PSBTBT/PC ${ }_{71} B M$ devices originates from the NGR under a short-circuit condition caused by charge accumulation. This suggests that not only a reduced Langevin context but also efficient charge extraction is necessary for achieving a high FF with a thick active layer.

Blends employing crystalline donor polymer exhibits relatively small reduction factor for both fullerene and NFA-based solar cells. Although it has been still under debate about the mechanism of the reduction of NGR caused by polymer crystallinity, several possible mechanisms have been proposed as will be described in detail in the part 'Origin of Slower NGR'.

\section{Nonfullerene Acceptor (NFA) -Based Solar Cells}

Nonfullerene acceptors (NFAs) have attracted considerable attention as an alternative to fullerenes by providing stronger optical absorption and allowing further optimisation of the molecular orbital energy landscape. The PCEs for NFA-based OPVs have been improved significantly up to $>18 \%$. However, the optimised active layers for NFA-based cells have been typically kept as thin as possible $(\sim 100$ $\mathrm{nm}$ ) to retain a high FF. Only limited cells have shown better performance even with a thick active layer. For example, Zheng et al. reported highly efficient NFA-based OPVs where they modified the hole-transporting layer to improve the FF. ${ }^{137}$ By mixing $\mathrm{WO}_{x}$ nanoparticles with PEDOT:PSS emulsion to obtain better interfacial properties, they achieved a boosted performance with an FF of 0.8079 and a PCE of $14.57 \%$ with a $110-n m$ thick active layer, which is one of the highest records for single-junction OPVs. The FF still remains over 0.7 with a $290-\mathrm{nm}$ thick active layer. They conducted contact angle measurement and found that the surface free energy increased with the introduction of $\mathrm{WO}_{x}$, improving active layer morphology on the hole transporting layer. A more balanced mobility and longer charge carrier lifetime were observed and considered to be the cause of higher FF. Therefore, morphology control is effective for reduced NGR and high FF in NFA-based OPVs. On the other hand, recent experimental results show that NGR is suppressed in blends employing fluorinated molecules. Zhang et al. found that PBDB-T- 
$2 F / I T-4 F$ solar cells exhibit a reduction factor no more than oneseventh of that in PBDB-T-2F/ITIC solar cells. ${ }^{127}$ Fluorination of molecules is reported to increase domain purity by enhancing inter/intramolecular interaction and to decrease Coulombic interaction of CTS by larger dielectric constant. ${ }^{138,139}$ Though the mechanism of reduced NGR is still unclear, fluorination can be one of strategies to suppress NGR loss.

NFAs based on n-type conjugated polymers such as F8BT are interesting candidates as an alternative to fullerenes as well. ${ }^{140,141}$ All-polymer blend solar cells routinely lagged in the PCE behind their fullerene-based analogues. This is mainly due to poor $J_{S C}$ and FF for all-polymer cells compared with the fullerene-based analogues. As mentioned above, free charge generation in all-polymer OPVs have been limited by severe GR loss. ${ }^{142-149}$ For example, Westenhoff et al. analysed the charge dynamics in a blend of PFB and F8BT by TAS. ${ }^{143}$ They found that $\sim 75 \%$ of transient signals decayed but their polarization anisotropy remained high. They therefore attributed this decay fraction to GR of CTSs to lower-lying triplet states of F8BT. Charge dissociation efficiency and the PCE for all-polymer cells have been improved gradually owing to development of better acceptor polymers such as P(NDI2OD-T2). ${ }^{150}$ A PCE of $>11 \%$ was reported in $2019 .{ }^{151}$ On the other hand, most all-polymer OPVs still have a serious problem with $F F$, which is typically $<0.6$. For example, Hwang et al. reported PBDTTT-CT/30PDI blend and PTB7-Th/PNDIS-HD blend solar cells, both of which exhibit a PCE of $>6 \%$ but an FF of $\sim 0.5 .{ }^{152,153}$ To date, very limited all-polymer OPVs exhibit an FF of $>0.7$ with a thick active layer. Schubert et al. reported an example where an FF of 0.7 was obtained with a $410-\mathrm{nm}$ thick active layer based on a blend of RR-P3HT as a donor and P(NDI2OD-T2) as an acceptor, while the PCE of this device was unfortunately poor ( 1\%). ${ }^{154}$ They found by TDCF measurements that NGR is three orders of magnitude lower than the Langevin recombination rate. ${ }^{133}$ They also suggested a strong correlation between domain purity and recombination kinetics where the strongly suppressed recombination rate can be obtained with highly pure domains.

The above discussion indicates that the common factor in blends where reduced NGR is observed is that the domain purity of donor and acceptor material is high for some reason. High crystallinity of polymer induces high domain purity, and fluorination of SMNFA enhances aggregation and then purity. Therefore, increasing domain purity by material design or fabrication condition would be effective for reduced NGR. However, we still need further studies to gain in-depth understanding of the reduced NGR.

\section{Origin of Slower NGR}

In some OPVs, NGR is reduced by $2-4$ orders of magnitude compared to Langevin theory, but there is no unified understanding of the phenomenon. Here, we will summarise recent researches about the origin of slower NGR. We also propose that an entropy effect, which decreases Coulomb attraction energy, would reduce NGR in crystalline or aggregated OPVs.

\section{“Effective mobility” Limited Recombination}

In the Langevin framework, spatially and energetically homogeneous charge transport is assumed. However, it would be not the case in $\mathrm{BHJ}$ OPVs. Koster et al. have proposed a simple recombination model based on the minimum mobility. ${ }^{155}$

$$
\gamma_{\min }=\frac{q \min \left(\mu_{\mathrm{e}}, \mu_{\mathrm{h}}\right)}{\varepsilon_{0} \varepsilon_{\mathrm{r}}}
$$

In this model, the total time for both electrons and holes to reach the interface is the limiting process of recombination. They confirmed the validity of this model by drift-diffusion simulation incorporating the model to reproduce $J-V$ characteristics of solar cells based on RR$\mathrm{P} 3 \mathrm{HT} / \mathrm{PC}_{61} \mathrm{BM}$ blend films annealed at different temperatures.

On the other hand, Groves et al. have studied the accuracy of this model by implementing kinetic Monte Carlo simulations. ${ }^{156}$ They examined the dependence of imbalanced mobility and found that while the recombination rate is well described by the Koster model when mobility is balanced, the recombination rate constant slightly depends upon the smallest mobility as shown in Figure 11 . They proposed that the deviation from the Koster model arises from the fact that charge carriers are spatially distributed with different distances from the interface.

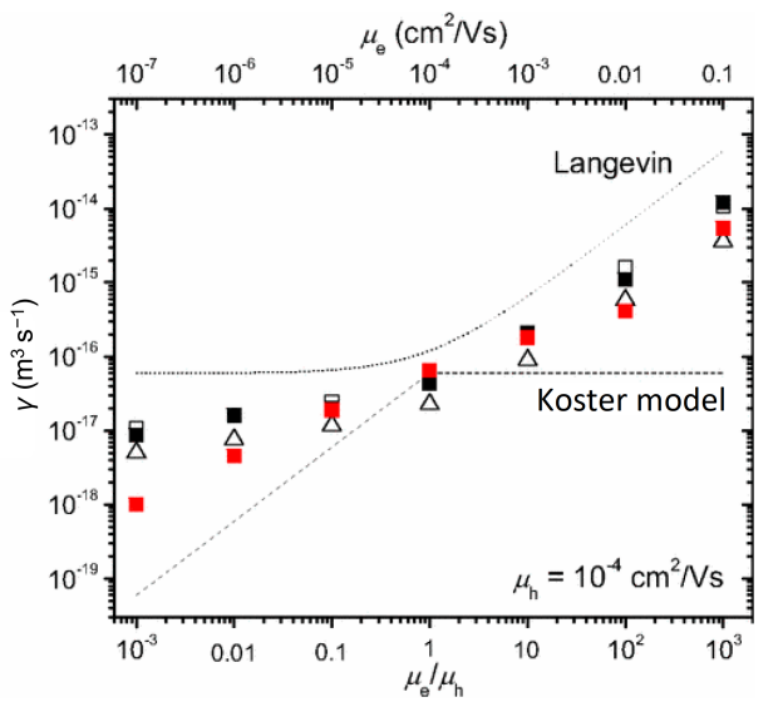

Figure 11. Log-log plots of recombination rate constant measured for a 1:1 blend of electron donor and acceptor polymers against the ratio of electron mobility to hole mobility (lower axis) and electron mobility (upper axis) with an average morphology feature domain size of $d=4$ (black) and $35 \mathrm{~nm}$ (red). The squares and triangles represent $\sigma=0$ and $75 \mathrm{meV}$, respectively, where $\sigma$ is standard deviation of a Gaussian distributed energetic disorder. The open and closed symbols represent $E=0$ and $10^{7} \mathrm{~V} \mathrm{~m}^{-1}$. In all cases $\mu_{\mathrm{h}}=10^{-4} \mathrm{~cm}^{2} \mathrm{~V}^{-1} \mathrm{~s}^{-1}$. The dotted and dashed lines represent the prediction by the Langevin equation and the Koster model, respectively. Adapted with permission from ref. 156. Copyright 2008 American Physical Society.

Heiber et al. have studied the effect of domain size $d$ in detail by kinetic Monte Carlo simulation and hence proposed a more general equation to explain their simulation results as follows 


$$
\gamma=\frac{q}{\varepsilon_{0} \varepsilon_{\mathrm{r}}} f(d) 2\left(\frac{\mu_{\mathrm{e}}^{g}+\mu_{\mathrm{h}}^{g}}{2}\right)^{1 / g}
$$

where $f(d)$ and $g$ are domain-size dependent prefactor and exponent, respectively. ${ }^{157}$ The result of the simulation is shown in Figure 12. For small domains with $d<10 \mathrm{~nm}, f(d)$ and $g$ are close to unity and hence Equation 37 corresponds to the Langevin model. For large domains with $d>35 \mathrm{~nm}$, on the other hand, $g$ continues to decrease and hence the minimum mobility effect emerges. For intermediate domains with $d$ of $10-35 \mathrm{~nm}, g$ approaches zero and the recombination is dependent on the geometric mean of mobility. These studies suggest that the actual recombination rate is somewhere in between the Langevin model and the Koster model, depending on the domain size. Coropceanu et al. also employed kinetic Monte Carlo simulation to study the impact of morphology and the lifetime of CTS on NGR dynamics. ${ }^{158}$ They considered four different morphologies including bilayers with flat and rough interfaces and BHJs with large $(25 \mathrm{~nm})$ and small $(5 \mathrm{~nm})$ average domain sizes (Figure 13a). As shown in Figure $13 \mathrm{~b}$, the NGR rate constant varied significantly depending on morphologies: the slower NGR rate was obtained with decreasing interfacial area-to-volume ratio. They also considered the impact of lifetime of CTS by modifying attempt frequency of recombination of CTS. The result suggests that long-lived CTS could reduce NGR.

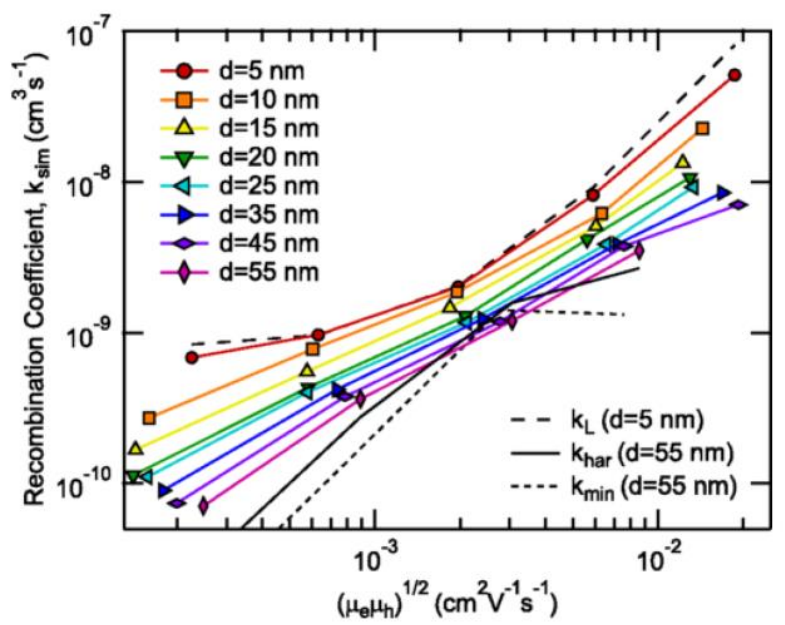

Figure 12. The result of kinetic Monte Carlo simulation of mobility dependence of NGR coefficient $\left(k_{\text {sim }}\right)$ in terms of the effect of the domain size compared to the Langevin $\left(k_{\mathrm{L}}\right)$, harmonic mean $\left(k_{\text {har }}\right)$, and the Koster $\left(k_{\text {min }}\right)$ models. Reprinted with permission from ref. 157. Copyright 2015 American Physical Society. (a)
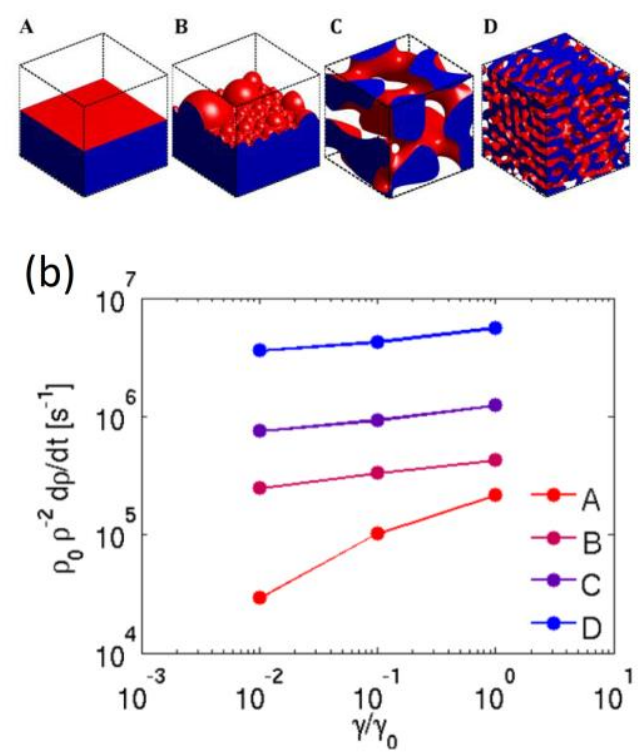

Figure 13. (a) Supposed morphologies of bilayers with flat $(A)$ and rough (B) interfaces and BHJs with large $(25 \mathrm{~nm}, \mathrm{C})$ and small ( 5 $\mathrm{nm}$, D) average domain sizes. (b) NGR rate constant plotted against the normalized attempt frequency. Reprinted with permission from ref. 158. Copyright 2017 American Chemical

\section{Concentration Gradients of Charges}

Deibel et al., explained the origin of reduced NGR with their own model considering concentration gradients of charge carriers. ${ }^{28}$ Since $\mathrm{OPV}$ is an ambipolar device with asymmetric contacts, there is concentration gradients of charge carriers in the device. As shown in Figure 14a, electron concentration would be large near the cathode, and the hole concentration would be large near the anode. Then, the local recombination rate is determined by the product of local concentration of electrons $(n(x))$ and that of holes $((p(x))$ where $x$ represents the distance from anode to cathode. In most measurements, on the other hand, spatially averaged concentration of charges $(\overline{\boldsymbol{n}(\boldsymbol{x})}$ or $\overline{\boldsymbol{p}(\boldsymbol{x})})$ are evaluated, leading to an overestimation of the recombination rate. Therefore, they described the Langevin reduction factor as

$$
\zeta=\frac{\frac{1}{L} \int_{0}^{L} n(x) p(x) \mathrm{d} x}{\overline{n(x)} \cdot \overline{p(x)}}
$$

They conducted drift-diffusion simulation and calculated local concentrations of electrons and holes to determine the reduction factor on the basis of Equation 38. Figure 14b shows the simulated reduction factor and experimental values reported by Deibel et al. ${ }^{113}$ and Juŝka et al. ${ }^{125}$ The temperature dependence of the experimental values are well reproduced with their model whereas other models cannot explain such temperature dependence. However, there is still a gap between the absolute magnitudes of simulated and experimental values by a factor of $1 / 200$ for the data of Juŝka and by a factor of $1 / 20$ for the data of Deibel. They considered the difference of the values as a constant component induced by other factors. Therefore, the actual value of the reduction factor may be 
(a)

$$
\text { our model: concentration }
$$

gradient in ambipolar device

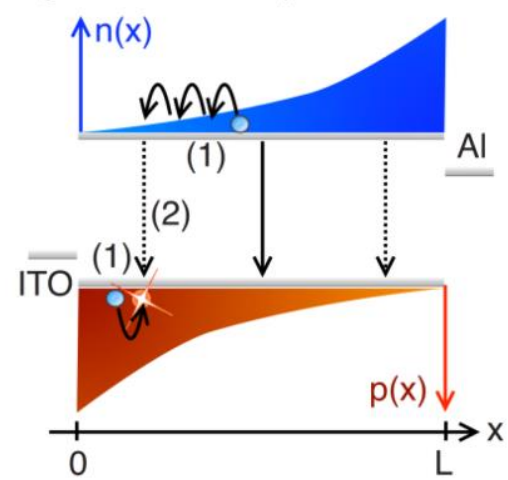

(b)

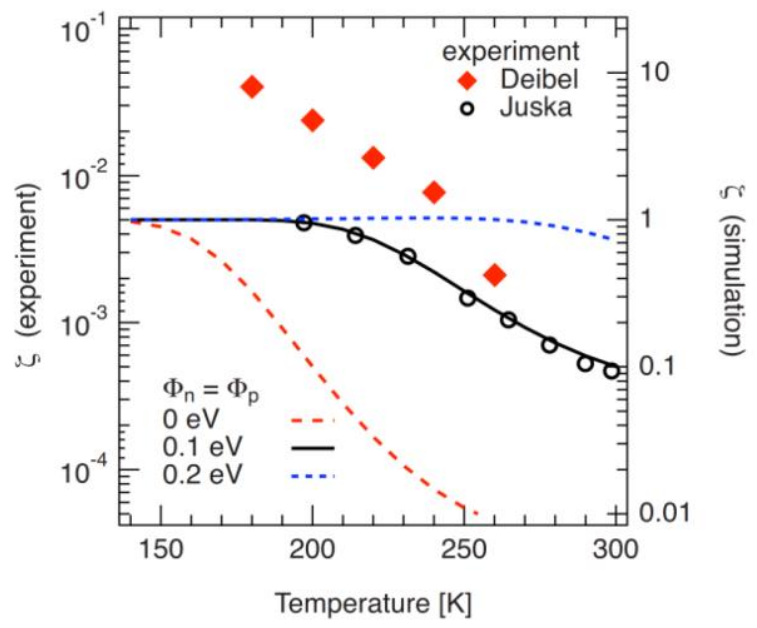

Figure 14. (a) Schematic representation of the model. $L$ is the thickness of a device and $n(x)$ and $p(x)$ are the positiondependent electron and hole density where $x$ is the distance from anode to cathode. (b) Simulated temperature dependence of reduction factor $\zeta$ with experimental values by photo-CELIV. $\Phi_{p}$ and $\Phi_{\mathrm{n}}$ represent injection barriers at the anode and at the cathode, respectively. Reprinted with permission from ref. 28. Copyright 2009 American Physical Society.

determined by a combination of this model and other factors mentioned in this section.

\section{D Langevin Recombination}

Juŝka et al. attributed the origin of reduced NGR to anisotropic transport of charges in crystalline polymers. ${ }^{30,} 159$ The time needed for charges to meet under influence of Coulomb interaction is expressed as

$$
t_{\mathrm{m}}=\int_{0}^{r_{\mathrm{m}}} \frac{\mathrm{d} r}{\left(\mu_{\mathrm{e}}+\mu_{\mathrm{h}}\right) E(r)}
$$

where $r_{\mathrm{m}}$ is an interaction radius and $E(r)$ represents Coulomb electric field.

$$
E(r)=\frac{e}{4 \pi \varepsilon \varepsilon_{0} r^{2}}
$$

In the classic Langevin model, charge recombination take place in 3D isotropic space, and the $r_{\mathrm{m}}$ is determined by $4 \pi r_{\mathrm{m}}{ }^{3} / 3=1 / n$. Therefore, 3D Langevin recombination probability for holes is given by

$$
f_{3 \mathrm{D}}=\frac{1}{t_{\mathrm{m}}}=\frac{e\left(\mu_{\mathrm{e}}+\mu_{\mathrm{h}}\right) n}{\varepsilon \varepsilon_{0}}
$$

On the other hand, the situation is quite different in blend films employing crystalline polymers. Crystalline polymers form lamellar structures, and charges are mainly transported along the 2D lamellar structure. It is reported that the mobility across and along the lamellar structure differs more than 100 times. Then, charge recombination dynamics is also limited by the $2 \mathrm{D}$ structure. When lamellar spacing $l \ll r_{\mathrm{m}}, r_{\mathrm{m}}$ is determined by $\pi r_{\mathrm{m}}{ }^{2} l=1 / n$. Thus, 2D Langevin recombination probability is given by

$$
f_{2 \mathrm{D}}=\frac{3 \sqrt{\pi}}{4} \frac{e\left(\mu_{\mathrm{e}}+\mu_{\mathrm{h}}\right)}{\varepsilon \varepsilon_{0}}(\ln )^{3 / 2}
$$

Using Equations 41 and 42, the Langevin reduction factor is expressed as

$$
\zeta=\frac{3 \sqrt{\pi}}{4} l^{3 / 2} n^{1 / 2}
$$

This indicates that the reduction factor is dependent on the lamellar spacing and charge density. The lamellar spacing of RR-P3HT is reported to be $1.6 \mathrm{~nm}$. Assuming $n=10^{16} \mathrm{~cm}^{-3}$, $\zeta$ of $6 \times 10^{-3}$ is obtained, and the value is close to experimental values for RR$\mathrm{P} 3 \mathrm{HT} / \mathrm{PC}_{61} \mathrm{BM}$ solar cells.

\section{CTS as Intermediates for NGR}

In the Langevin framework, in which electron-hole recombination occurs when the separation distance between them reaches zero, intermediate states that may be formed when electron and hole come close are not taken into account. However, this would not be the case for OPVs. An encounter of electron and hole leads to the formation of a CTS as evidenced by the observation of electroluminescence (EL) from the CTS. Figure 15a shows the EL spectra of an MDMO-PPV/PC ${ }_{61} \mathrm{BM}$ blend solar cell. ${ }^{160} \mathrm{~A}$ clear luminescence observed in the near-IR region is attributed to emission from the CTS generated via charge recombination. The fact that the CTS acts as an intermediate for NGR indicates that redissociation of these CTS allows for additional opportunities for charges to be collected before charges completely deactivate to the GS. This situation is schematically depicted in Figure 15b. In active layers, electrons and holes encounter with a rate constant of $\gamma_{\mathrm{L}}$, forming a CTS. Once the CTS is formed, they will decay or dissociate with rate constants of $k_{\text {rec }}$ and $k_{\text {dis }}$, respectively. The rate equations can be written as

$$
\begin{gathered}
\frac{\mathrm{d} n}{\mathrm{~d} t}=-\gamma_{\mathrm{L}} n p+k_{\mathrm{dis}} n_{\mathrm{CT}} \\
\frac{\mathrm{d} n_{\mathrm{CT}}}{\mathrm{d} t}=\gamma_{\mathrm{L}} n p-\left(k_{\mathrm{dis}}+k_{\mathrm{rec}}\right) n_{\mathrm{CT}}
\end{gathered}
$$

where $n_{\mathrm{CT}}$ is the density of CTS. ${ }^{61}$ Under steady-state approximation, we can assume $\mathrm{d} n_{\mathrm{CT}} / \mathrm{d} t=0$. Then $n_{\mathrm{CT}}$ is expressed as 
(a)
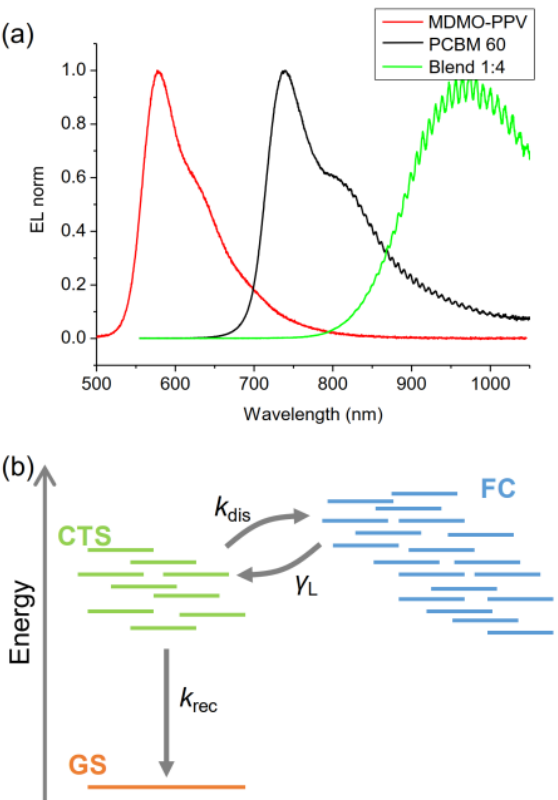

Figure 15. (a) Normalised electroluminescence spectra of pristine MDMO-PPV, $\mathrm{PC}_{61} \mathrm{BM}$, and the blend. Adapted with permission from ref. 160. Copyright 2009 American Chemical Society. (b) Kinetic scheme showing reduced Langevin recombination in OPVs. FC, CTS, and GS represent free charge carrier, charge transfer state, and ground state, respectively. Electrons and holes encounter with a rate constant of $\gamma_{\mathrm{L}}$, forming a CTS. The CTS then recombine or re-dissociate with rate constants of $k_{\text {rec }}$ or $k_{\text {dis, }}$

$$
n_{\mathrm{CT}}=\frac{\gamma_{\mathrm{L}}}{k_{\mathrm{dis}}+k_{\mathrm{rec}}} \boldsymbol{n p}
$$

Using this relation, Equation 44 turns into

$$
\frac{\mathrm{d} n}{\mathrm{~d} t}=-\frac{k_{\mathrm{rec}}}{k_{\mathrm{dis}}+k_{\mathrm{rec}}} \gamma_{\mathrm{L}} n p
$$

This means that the reduction factor is given by

$$
\zeta=\frac{\boldsymbol{k}_{\mathrm{rec}}}{\boldsymbol{k}_{\mathrm{rec}}+\boldsymbol{k}_{\mathrm{dis}}} .
$$

The dissociation efficiency from CTS is kinetically given by $\eta_{\text {CTD }}=$ $k_{\text {dis }} /\left(k_{\text {rec }}+k_{\text {dis }}\right)$. Thus, the reduction factor is given by $1-\eta_{\text {CTD }}{ }^{93,161}$ This clearly tells us that the more the CTS can re-dissociate to free charges the smaller the reduction factor $\zeta$ will be. Note that the overall charge dissociation efficiency $\eta_{\text {CD }}$ might be different from $\eta_{\text {CTD }}$ if free charge carriers are generated not only from a relaxed CTS but also from a hot CTS. As will be discussed later, re-dissociation of the CTS significantly depends on various factors such as crystallinity, domain purity, and electron spin.

\section{Equilibrium between CTS and FC}

Burke et al. proposed that if the NGR is reduced, there should be a thermal equilibrium between a population of CTS and FC. ${ }^{162}$ They showed that the density of CTS at the open-circuit voltage is given by

$$
N_{\mathrm{CT}}=f N_{0} \exp \left(\frac{\sigma_{\mathrm{CT}}^{2}}{2\left(k_{\mathrm{B}} T\right)^{2}}\right) \exp \left(\frac{q V_{\mathrm{OC}}-E_{\mathrm{CT}}}{k_{\mathrm{B}} T}\right) .
$$

where $f$ is the volume fraction related to the mixing ratio, $N_{0}$ is the effective density of states, $\sigma_{\mathrm{CT}}$ is the standard deviation of the CTS energetic distribution, and $E_{\mathrm{CT}}$ is the energy of CTS. Inspired by the fact that the CTS is in equilibrium with free carriers, Liu et al. directly correlated the reduction factor with the energetic and dynamic behaviour of the CTS. ${ }^{163}$ As a result, the reduction factor is given by

$$
\zeta=\frac{f \exp \left(\frac{\sigma_{\mathrm{CT}}^{2}}{2\left(k_{\mathrm{B}} T\right)^{2}}\right)}{\gamma_{\mathrm{L}} N_{0} \tau_{\mathrm{CT}}} \exp \left(\frac{E_{\mathrm{b}}}{k_{\mathrm{B}} T}\right) .
$$

where $E_{\mathrm{b}}$ is the binding energy of the CTS and $\tau_{\mathrm{CT}}$ is the characteristic time with which the CTS decays to the GS. Note that $\tau_{\mathrm{CT}}$ is not the overall CT lifetime but is given by $1 / k_{\text {rec }}$. This equation shows that longer $\tau_{\mathrm{CT}}$ would give a smaller reduction factor because the other parameters are independent on $\tau_{\mathrm{CT}}$. In most cases, $\tau_{\mathrm{CT}}$ is mainly dependent on nonradiative recombination rate rather than radiative recombination rate because of low quantum yield of CT emission. Thus, suppressing nonradiative recombination would result in smaller reduction factors. They also showed that the temperature dependence of the reduction factor derived from their CT model is consistent with that derived by the analytical continuum approach of Hilczer and Tachiya. ${ }^{29}$ Note that the equation can explain such qualitative trends, but cannot give quantitative values with reasonable energetic and kinetic parameters.

\section{Role of Electron Spin for NGR}

Electron spin plays an important role in organic light-emitting diodes (OLEDs). As the spins of injected charges are completely uncorrelated recombination of electrons and holes gives singlet and triplet excitons with a ratio of 1:3 according to simple spin statistics. On the other hand, the importance of spin was not experimentally demonstrated in the OPV community until Rao et al. reported. ${ }^{164,165}$ They demonstrated that NGR leads to the formation of both singlet and triplet CTS ( ${ }^{1} \mathrm{CTS}$ and ${ }^{3} \mathrm{CTS}$ ) as schematically shown in Figure 16.

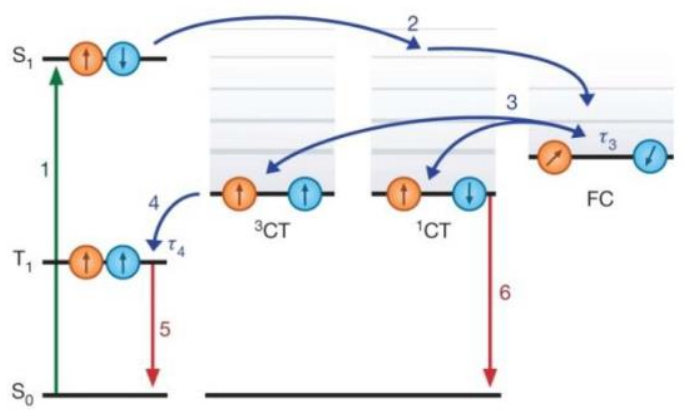

Figure 16. Kinetic scheme showing spin dependent charge recombination. Encounter of electrons and holes lead ${ }^{1} \mathrm{CTS}$ and ${ }^{3} \mathrm{CTS}$ with a ratio of $1: 3 .{ }^{1} \mathrm{CTS}$ then recombine to the GS or re-dissociate to free carriers. ${ }^{3} \mathrm{CTS}$ then recombine to triplet exciton states or re-dissociate to free carriers. The rate constants could be dependent on spin of CTS. Reprinted with permission from ref. 164. Copyright 2013 Springer Nature. 

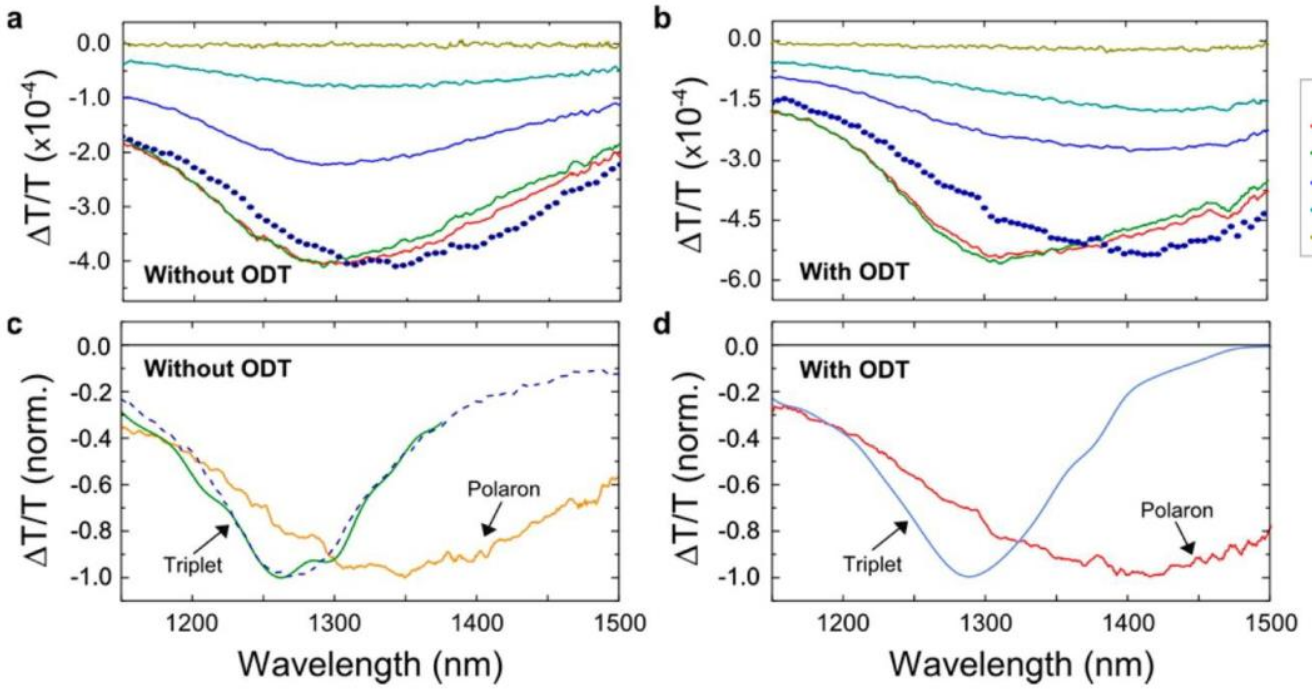

Figure 17. TA spectra in the near IR region of PCPDTBT/PC ${ }_{71} B M(1: 2)$ blend films processed without (a) and with (b) a solvent additive of 1,8-octanedithiol (ODT), excited at $532 \mathrm{~nm}$ with a fluence of $2.4 \mu \mathrm{J} \mathrm{cm}^{-2}$. The solid lines represent the spectral evolution from $1 \mathrm{~ns}$, where the samples were excited by electrically delayed pulses with a pulse width of $1 \mathrm{~ns}$. The filled circles represent the spectra measured at 100 ps (using optically delayed pulses with a pulse width of $200 \mathrm{fs}$ ). (c,d) Normalised polaron and triplet absorption spectra in the respective blend films, which were deconvoluted from the spectra shown in (a) and (b). The blue dashed line in (c) represents the triplet absorption spectra directly measured in a pristine PCPDTBT thin film (at a delay time of $50 \mathrm{~ns}$ ). The overall blue shift observed in both blends was due to the emergence of triplet absorption on a nanosecond time scale. Reprinted with permission from ref. 165. Copyright 2014 American Chemical Society.

The ${ }^{3} \mathrm{CTS}$, then, recombines to polymer triplet excitons while ${ }^{1} \mathrm{CTS}$ recombines to the GS. The back charge transfer from polymer triplet excitons to the ${ }^{3}$ CTS is usually energetically unfavourable. Rather, triplet excitons quickly annihilate with charges, indicating that recombination to triplet excitons is a terminal loss process in OPVs. They also found that polymer triplet excitons were clearly observed only in blends with disordered $\mathrm{PC}_{71} \mathrm{BM}$ or other noncrystalline fullerene derivatives such as ICMA and ICBA (Figure 17). In a blend with ordered $\mathrm{PC}_{71} \mathrm{BM}$ aggregates, triplets were only observed at a low temperature. These findings suggest that a thermally activated process competes with the formation of triplets from the ${ }^{3} \mathrm{CTS}$, which is most probably due to re-dissociation of the ${ }^{3} \mathrm{CTS}$ to free carriers. Menke et al. took the idea one step further. ${ }^{18}$ They expected that once the ${ }^{1} \mathrm{CTS}$ and ${ }^{3} \mathrm{CTS}$ are formed, they will have individual decay and re-dissociation rates. From TAS measurements, they successfully estimated decay probability of ${ }^{1} \mathrm{CTS}$ and ${ }^{3} \mathrm{CTS}$ in a PIPCP/PC ${ }_{61} \mathrm{BM}$ blend. For the PIPCP/PC 61 BM blend, the ${ }^{3} \mathrm{CTS}$ is three times more likely to decay than the ${ }^{1} \mathrm{CTS}$, suggesting that it is important to reduce the rate of energy transfer to triplet state for further improvement of this system. Armin et al. demonstrated the importance of ${ }^{3} \mathrm{CTS}$ for reduced NGR. ${ }^{166}$ They solved kinetic rate equations for a model considering the role of ${ }^{3} \mathrm{CTS}$ and calculated reduction factor. The result suggested that very small reduction factor is achieved only in the presence of both slow energy transfer from ${ }^{3} \mathrm{CTS}$ to triplet state and fast dissociation of CTS.

\section{Energy Cascade near the Interface}

The cascade energy landscape at the donor/acceptor interface is thought to play a crucial role in charge carrier dynamics. In $\mathrm{BHJ}$ blend films of a crystalline polymer and small molecule acceptor such as PCBM, there would be three phases, pure polymer crystalline phase, pure acceptor aggregated phase, and mixed amorphous phase. Then, holes and electrons are energetically more stable in each pure phase of donor and acceptor, respectively. Thus, these three phases form cascaded energy structure. ${ }^{167,168}$

Such a spatial separation of carriers could suppress NGR and hence improve charge collection efficiency. Burke et al. calculated the charge carrier lifetime in the three-phase $\mathrm{BHJ}$ with and without cascade energy landscape by assuming the thermal equilibrium between CTS and FC as mentioned above. ${ }^{162}$ They reached the conclusion that, with the aid of an energetic cascade, the charge carrier lifetime becomes longer than that without an energetic cascade, indicating reduced NGR. On the other hand, Bartelt et al. employed a 1D drift-diffusion device simulation and calculated the charge carrier mobility required to achieve an FF of 0.8 in a $300-n m$ thick solar cells. ${ }^{169}$ The results showed that the required mobility is directly proportional to the NGR rate constant. Therefore, a spatial separation of charges via cascade energy structure would suppress NGR and promote charge collection, leading to a high FF even with a thick active layer.

The energy shift between the phases was experimentally evaluated by Sweetnam et al. who combined cyclic voltammetry (CV) and ultraviolet photoelectron spectroscopy (UPS). ${ }^{168}$ The shifts of valence bands of polymer were found to be induced by two factors including bandgap widening associated with the changes in a polymer conjugation length and intermolecular interactions between polymer and fullerene. The total energy shift was in the order of 300 meV. They stated that a large cascade should push charges away 
(a)

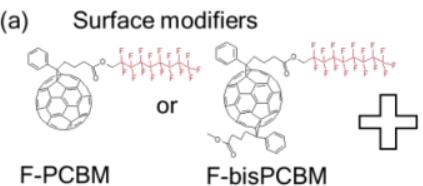

Bulk materials

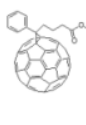

PCBM

or

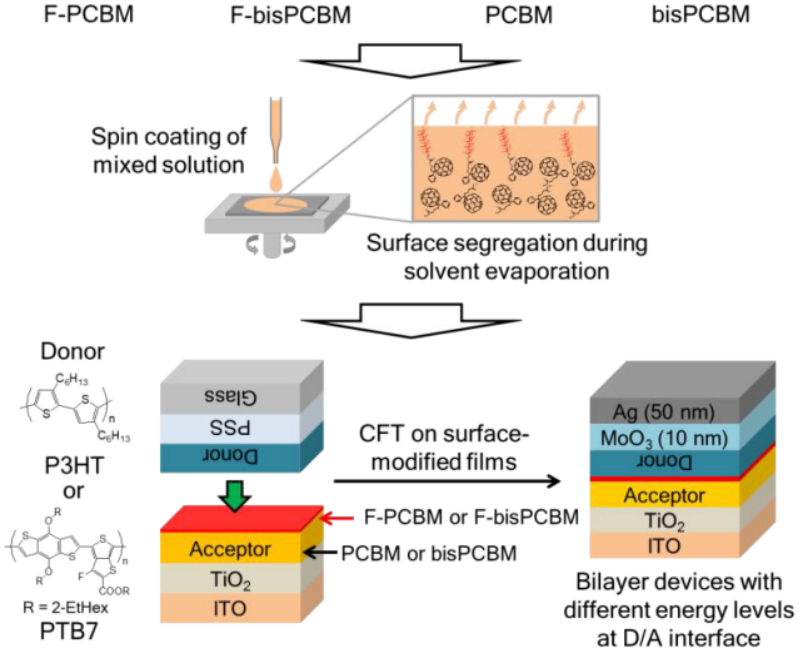

(b)
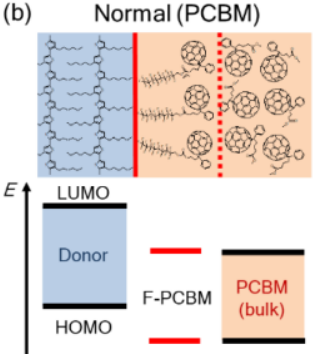

Normal (bisPCBM)

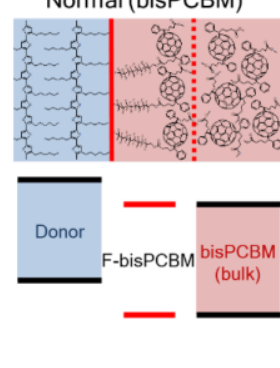

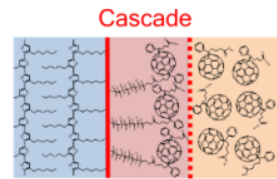

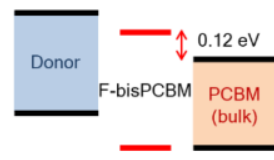

Trap

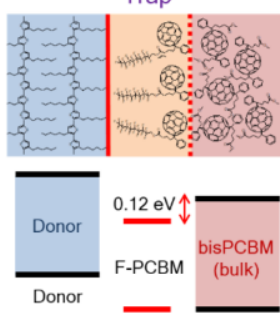

Figure 18. (a) Chemical structures of the surface modifiers and the bulk materials. The bilayer devices were fabricated by CFT method after the surface modification by SSM. (b) Four different energy level alignments at the D/A interface in the bilayer OPVs. Reprinted with permission from ref. 194. Copyright 2015 WILEY-VCH Verlag Gmbh \& Co. KGaA, Weinheim.

from interface and reduce recombination loss. Such a hole transfer due to the energy cascade have been directly observed by spectroscopic techniques. Guo et al. studied exciton and charge dynamics in RR-P3HT/PC ${ }_{61} B M$ blend films by TAS and showed that polymer polarons migrate from amorphous to crystalline domain with a time constant of several hundreds of picoseconds. ${ }^{44}$ Gallaher et al. also found hole transfer from intermixed to pure polymer region by TAS for blends based on kinds of polymers with different curvatures of backbone. ${ }^{170}$ They suggested that good miscibility with $\mathrm{PC}_{61} \mathrm{BM}$ due to high curvature leads to the formation of the threephase structure and hole migration.

The relation between the magnitude of energy shift and charge dynamics was studied by Groves. ${ }^{171}$ He conducted kinetic Monte Carlo simulation to investigate the effect of cascade energy structure on charge separation efficiency $\eta_{\mathrm{GS}}$. Assuming that the thickness of the cascade layer was $1 \mathrm{~nm}$, when the energy shift of donor HOMO $\Delta E_{\text {номо,D }}$ were changed from 0 to $300 \mathrm{meV}$, significant improvement of $\eta_{\mathrm{GS}}$ and its field dependence were observed. While $\eta_{\mathrm{GS}}$ was heavily field dependent without energy cascade, $\eta_{\mathrm{GS}}$ was larger than $\sim 0.9$ under wide range of operating field condition with $\Delta E_{\mathrm{DH}}=300 \mathrm{meV}$. This suggests that the experimentally observed energy shift on the

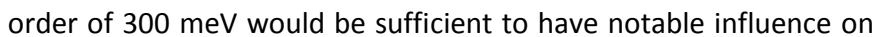
the charge separation efficiency $\eta_{\mathrm{GS}}$ and hence the reduction factor $\zeta$ as mentioned before. Indeed, charge carriers with longer lifetimes have been reported for ternary polymer solar cells with cascade energy structure as will be described below.

\section{Ternary Polymer Solar Cells}

The cascade energy structures can be more easily designed in ternary blend solar cells, in which three different materials are composed, by using appropriated materials combinations. More importantly, ternary blend solar cells can boost the light-harvesting efficiency because of complementary absorption bands. As such, they have been one of the hottest topics in this research field ${ }^{172-175}$ since Ohkita and his co-workers have successfully demonstrated improvements of the PCE in ternary blend devices. ${ }^{46,176-191}$ Ternary blend solar cells can be categorised into two types. One type relies on two parallel diodes where two donors (or two acceptors) work with a common acceptor (donor) independently. Another type has a similar fashion with dyesensitised solar cells. In the sensitised OPVs, the third component should be located at an interface between donor and acceptor materials, where the cascade HOMO and LUMO energy landscape boosts photovoltaic conversion. Thus, it is important to control the location of the third component in blend films. ${ }^{192}$ They reported sensitized OPVs where adding only a few wt\% of phthalocyanine (SiPc) or naphthalocyanine (SiNc) dyes to RR-P3HT/PC ${ }_{61} \mathrm{BM}$ blends leads to enhancement of the PCE up to $4.8 \%{ }^{46,176,177,184}$ This enhancement is due mainly to expansion of the absorption window by adding near-IR-absorbing dyes and more efficient exciton collection to the interface through Förster energy transfer from RRP3HT to near-IR dyes. They have also shown that ternary blend solar cells have potential to suppress NGR. ${ }^{184}$ More specifically, the lifetime of hole polarons in the RR-P3HT/SiPcBz6/PC ${ }_{61} B M$ ternary blends became three times longer than in RR-P3HT/PC ${ }_{61} B M$ binary blends by adding $15 \mathrm{wt} \%$ SiPcBz6 dye. This is because of the artificial cascade energy landscape constructed by the three components. Indeed, such artificial energy cascades do affect charge lifetime as Tan et al. demonstrated. ${ }^{193}$ They prepared a bilayer device based on RR-P3HT and P(NDI2OD-T2) and various trilayer devices where PDCBT acts as an interlayer between RR-P3HT and P(NDI2OD-T2). As HOMO and LUMO levels of PDCBT are in between that of RR-P3HT 
and $\mathrm{P}(\mathrm{NDI} 2 \mathrm{OD}-\mathrm{T} 2)$, those polymers form cascade energy landscape at the interface. They found that charge lifetime increased with the presence of thin ( 3-4 nm) PDCBT interlayer because it prevents charges from getting close by the same analogy as that mentioned above.

Izawa et al. have designed artificial interface cascade energy structures ${ }^{194}$ combining their contact film transfer (CFT) method ${ }^{195}$ and self-organised surface-segregated monolayer (SSM). ${ }^{196}$ Using these methods, they constructed well-organised model planar heterojunctions as shown in Figure 18a. They found that the cascade device shows higher $V_{\mathrm{OC}}$ and $\mathrm{FF}$ while lower $J_{\mathrm{SC}}, V_{\mathrm{OC}}$, and FF in the trap device compared to the normal device. Temperature dependence of $J_{S C}$ suggests that the activation energy of charge generation is negligible in the cascade device while the activation energy in the trap device is larger than the normal device. On the other hand, as shown in Figure 19, charge carrier lifetime of the cascade device evaluated from TPV/TPC measurements was longer than that of the normal device whereas that of the trap device exhibited no change. They concluded that the cascade energy landscape lowers the Coulomb binding energy at the interface and reduce both GR and NGR loss, and that controlling interface structure is essential for highperformance OPVs.
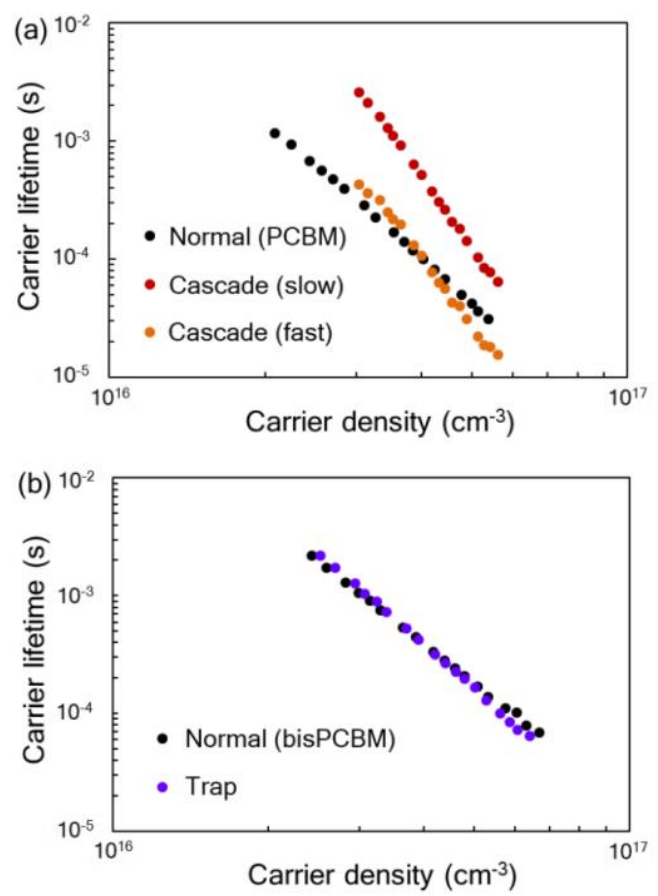

Figure 19. Log-log plots of charge carrier lifetime of the four bilayer OPV devices against charge carrier density. Reprinted with permission from ref. 194. Copyright 2015 WILEY-VCH Verlag Gmbh \& Co. KGaA, Weinheim.

Gasparini et al. have reported one of the highest FF of 0.77 for a ternary blend of PTB7/PSBTBT/PC ${ }_{71} \mathrm{BM}$ with a blend ratio of 0.85:0.15:1.5. ${ }^{197}$ Interestingly, PSBTBT contributes to efficient hole transport counterintuitive to the above sensitisation concept where holes shift from sensitiser to host donor. Rather, this blend might be a parallel-type ternary system. As shown in Figure 20, their photo-
CELIV measurements showed that adding a small amount (6\%) of PSBTBT improves charge mobility up to $28 \%$. At the same time, charge carrier lifetimes evaluated by TPV and CE measurements were enhanced up to $32 \%$ in the presence of PSBTBT. These enhancements result in efficient hole transport and a record FF of 0.77 .
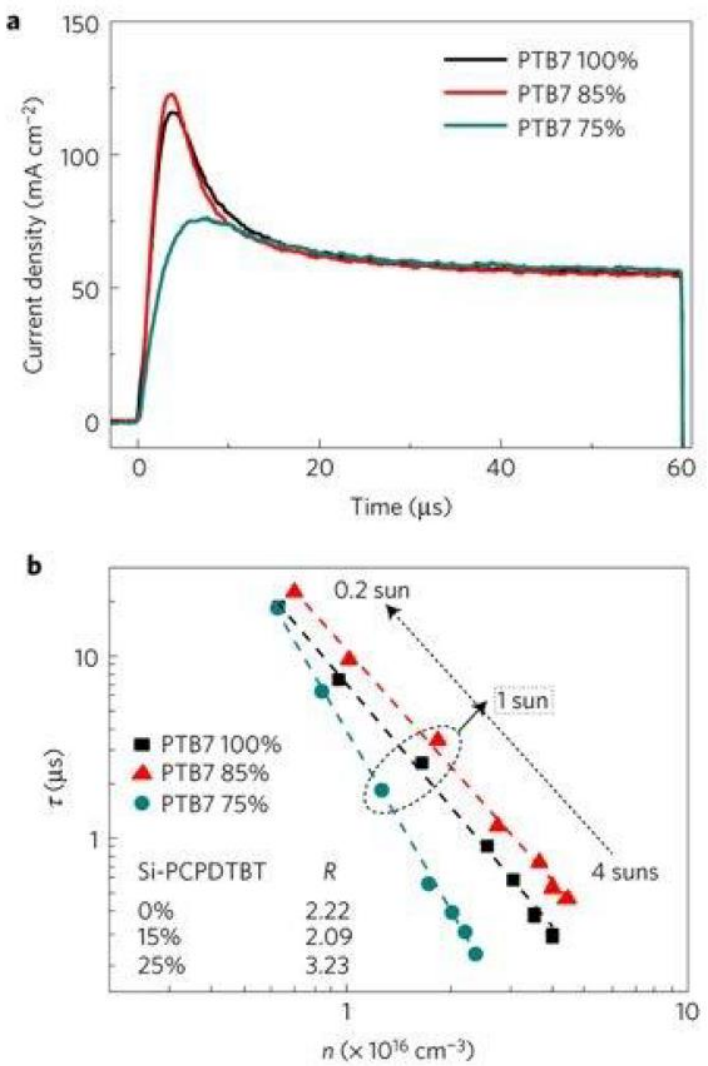

Figure 20. (a) Photo-CELIV transients of (black) PTB7/PC ${ }_{71} B M$, (red) $\mathrm{PTB7} / \mathrm{PSBTBT} / \mathrm{PC}_{71} \mathrm{BM}$ (0.85:0.15:1.5), and (green) PTB7/PSBTBT/PC ${ }_{71}$ BM (0.75:0.25:1.5) blend films exited at 780 $\mathrm{nm}$. All the transient measurements were recorded applying linearly increasing reverse bias pulse from 0 to $2 \mathrm{~V}$ for $60 \mathrm{~s}$ at a delay time $t_{\mathrm{d}}$ of $1 \mu \mathrm{s}$. (b) Log-log plots of charge carrier lifetime against charge carrier density measured by TPV/CE measurements. The inset table shows the NGR reaction order in these blend films. The dashed lines represent fitting lines. Reprinted with permission from ref. 197. Copyright 2016 Springer Nature.

Baran et al. have reported an interesting NFA-based ternary solar cell consisting of RR-P3HT as a donor and IDTBR and IDFBR as acceptors. ${ }^{198}$ The best performance was achieved with a blend ratio of 1:0.7:0.3 (RR-P3HT:IDTBR:IDFBR), giving a $J_{S C}$ of $14.4 \mathrm{~mA} \mathrm{~cm}^{-2}$, an FF of 0.64 , and a $V_{O C}$ of $0.82 \mathrm{~V}$. Importantly, this blend can retain relatively high FF values at an active layer thickness of $\sim 200 \mathrm{~nm}$. They performed optoelectronic measurements to investigate the effect of IDFBR addition on charge recombination. As shown in Figure 21, the carrier lifetime of the ternary blend was estimated to be $17 \mu \mathrm{s}$, which is two to three times longer than that for the binary analogues. Although the electron transport is slightly degraded by the addition 


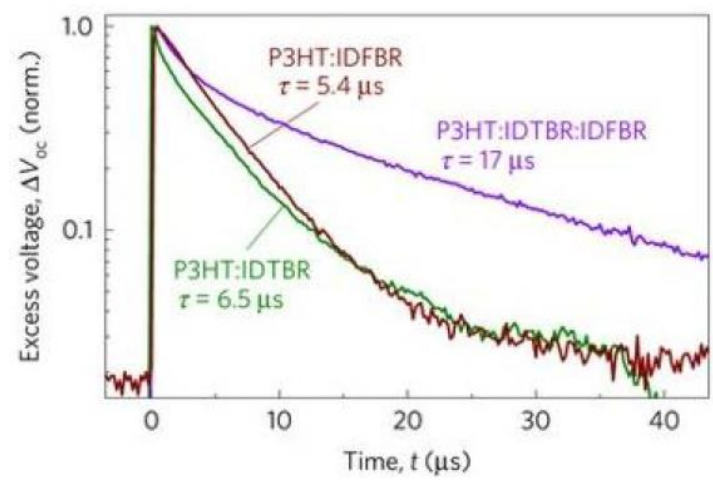

Figure 21. TPV transients for RR-P3HT/IDFBR/IDTBR ternary solar cells and their binary analogues. Reprinted with permission from ref. 198. Copyright 2016 Springer Nature.

of IDFBR because of increased electron trap density, the large enhancement in carrier lifetime cannot be rationalised by the small change in the electron mobility. Rather, it can be rationalised as a result of the cascade energy landscape at the interface. Because IDFBR exhibits a smaller electron affinity than IDTBR, and is more miscible with RR-P3HT, IDFBR is likely to locate at the interface between RR-P3HT and IDTBR, forming an energy cascade. The RRP3HT/IDFBR interface acts as an energetic barrier for electrons in the IDTBR domains to recombine with RR-P3HT holes. This increased lifetime is likely to contribute to a further $30-40 \mathrm{mV}$ increase in $V_{\text {oc }}$ of the ternary device.

\section{Entropy Effect}

Reduced NGR has been observed for OPVs where one of photovoltaic materials at least is crystalline or aggregated ones as summarised in Table 1. In such crystalline or aggregated blends, there would be pure donor, pure acceptor, and donor/acceptor mixed phases. As a result, a cascaded energy structure also is likely to be formed, which would be beneficial for reduced NGR as described before. On the other hand, such pure domains provide the greater number of charge states, which would play a key role in the entropy effect mentioned in the section of Fundamentals of NGR. This entropy effect has been discussed for efficient charge dissociation. Durrant et al. suggested that the entropy term would be comparable to the Coulomb interaction energy in ref. 41. In their review, ${ }^{42}$ they clearly demonstrated how the Coulomb attraction energy is reduced by considering the entropy contributions to the overall interfacial energetics. Figure 22 shows the overall free energy including entropy contribution as a function of separation distance of electron-hole pairs. Such a reduced Coulomb attraction energy would be beneficial for efficient charge dissociation. Later, Gregg has discussed this entropy effect on charge separation in terms of the dimensionality of organic photovoltaic materials. ${ }^{199} \mathrm{He}$ suggested that higherdimensional materials lead to larger entropy effects and hence have inherent advantages for charge separation. He also pointed out that the entropy effects are highest in equilibrium systems but decrease and become time-dependent in illuminated OPVs. We therefore think that this would be more effective for NGR in thermal equilibrium. For the NGR, the Coulombic attractive potential would

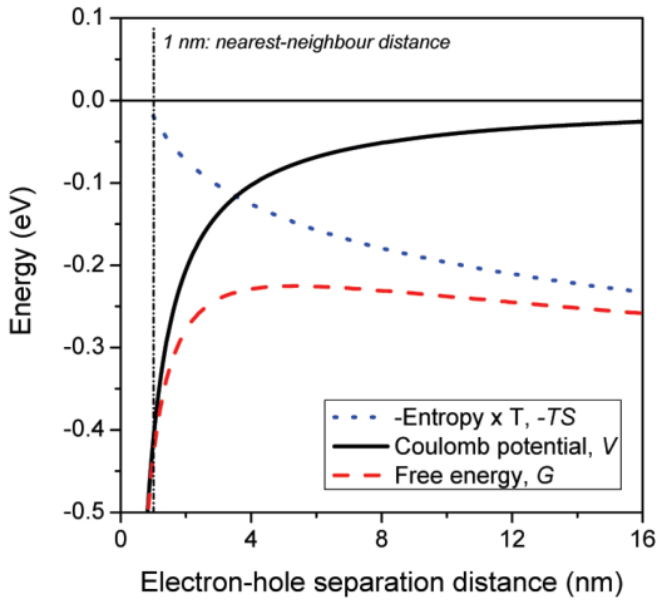

Figure 22. Calculated Coulomb potential energy, the entropy contribution to the free energy $\left(-T S=-k_{\mathrm{B}} T \ln W\right)$, where $W$ is the electronic degeneracy, and the free energy ( $G=H-T S$ ) of electron-hole pairs at a typical donor/acceptor interface as a function of electron-hole separation distance. For simplicity the authors assume the hole to be localized at the interface and only take the electron movement into consideration. The calculations were conducted with $\varepsilon_{\mathrm{r}}=3.5$ and $T=298 \mathrm{~K}$. They assumed $W$ as the number of $C_{60}$ species in the volume of a hemisphere extending from the interface and that each $\mathrm{C}_{60}$ molecule occupies $1 \mathrm{~nm}^{3}$. Reprinted with permission from ref. 42. Copyright 2010 American Chemical Society.

change to dissociative one by considering the entropy effect as shown in Figure 22. We therefore propose that the entropy effect would effectively reduce NGR in crystalline or aggregated OPVs.

\section{Relevance to Device Parameters}

\section{NGR Controls J-V Characteristics}

As schematically shown in Figure 23a, the observed current density $J$, as a function of applied voltage $V$, is a superposition of the generation current density $J_{\text {gen }}(V)$ and the loss current density $J_{\text {loss }}(V) \cdot{ }^{74,76}$

$$
J(V)=J_{\text {gen }}(V)+J_{\text {loss }}(V)
$$

Previous studies have shown that charge generation in highly efficient OPVs depends little on the external electric field, meaning that $J_{\text {gen }}$ can be assumed to be independent of $V$. On the other hand, $J_{\text {loss }}$ potentially comprises both GR and NGR, however, as mentioned above, GR loss is not significant in highly efficient OPVs. This means that the $J-V$ characteristics, or namely FFs, of OPVs are controlled by NGR as follows

$$
J(V)=J_{\mathrm{gen}}-q d R=J_{\mathrm{gen}}-q d \frac{n(V)}{\tau_{n}}
$$

where $d, \tau_{n}$ and $n(V)$ are the active layer thickness, experimentally determined charge carrier lifetime defined as $\mathrm{d} n / \mathrm{d} t=-n / \tau_{n}$, and voltage-dependent charge carrier density, respectively. Based on this idea, Maurano et al. successfully reproduced the $J-V$ curves of OPVs. ${ }^{74}$ Figure $23 \mathrm{~b}$ shows the experimental (lines) and calculated 
(a)

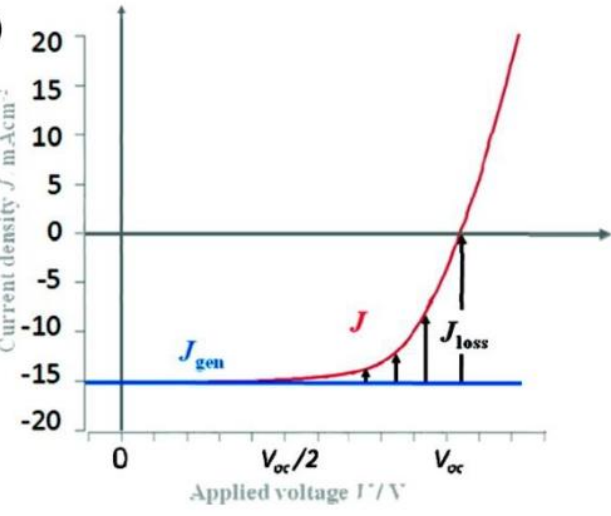

(b)

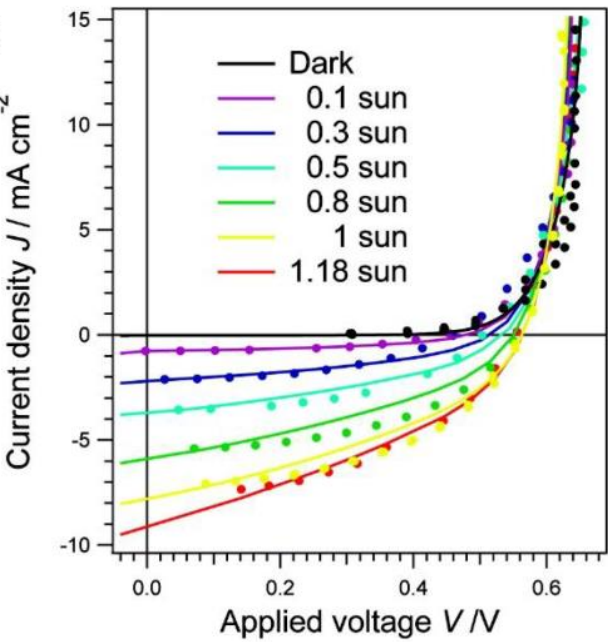

Figure 23. (a) The net current flow under illumination $J$ can be decomposed into a current due to the charge generation $J_{\text {gen }}$ and loss current due to the recombination Jloss. (b) Experimental $J-V$ curves (lines) as a function of light intensity between dark and 1.2 sun for a $\mathrm{P} 3 \mathrm{HS} / \mathrm{PC}_{61} \mathrm{BM}$ solar cell and calculated current density (circles) with $J_{\text {loss }}$ being determined by the NGR current. The current density was calculated considering external series resistance losses by $V_{\text {cell }}=V_{\text {app }}-$ $I_{\mathrm{s}}$. Reprinted with permission from ref. 74. Copyright 2011 American Chemical Society.

(circles) $J-V$ curves. The calculated data showed reasonably good agreement with the measured $J-V$ curves. This agreement provides undoubtable evidence that NGR is a dominant loss process that controls the $J-V$ characteristics.

Credgington and Durrant have successfully predicted $V_{\text {ocs }}$ of various blends from the NGR dynamics. ${ }^{77}$ At open circuit, the net current flow is zero, therefore, Equation 51 becomes

$$
J_{\text {gen }}=-J_{\text {loss }}
$$

In the range for which the above discussion is valid, $J_{\text {loss }}$ can be expressed as

$$
J_{\text {loss }}=-\frac{q d n}{\tau_{0} n^{-\lambda}}=-q d \frac{n_{0}^{1+\lambda}}{\tau_{0}} \exp \left(\frac{q(1+\lambda) V_{\mathrm{OC}}}{m k_{\mathrm{B}} T}\right)
$$

Assuming $J_{\text {gen }}$ is equal to $J_{\text {SC, }}$, and introducing a new variable $J_{0}, V_{\text {OC }}$ is expressed as an analogue to the Shockley equation for a $p$-n junction as follows.

$$
\begin{gathered}
V_{\mathrm{OC}}=\frac{n_{\mathrm{id}} k_{\mathrm{B}} T}{q} \ln \left(\frac{J_{\mathrm{SC}}}{J_{0}}\right) \\
J_{0}=q d \frac{n_{0}^{1+\lambda}}{\tau_{0}} \\
n_{\mathrm{id}}=\frac{m}{1+\lambda}
\end{gathered}
$$

Note that Equation 57 leads to Equation 6 because $1+\lambda$ is equal to $\delta$. Figure 24 shows the calculated $V_{\text {ocs }}$ of various blends based on their TPV/TPC measurements plotted against experimental values. Except for some small molecule systems for which the assumption of constant $J_{\text {gen }}$ is invalid, $V_{\text {OCS }}$ are well reproduced with an average deviation of less than $5 \mathrm{mV}$ without any free parameters. A voltage loss attributed to charge recombination differed by up to $200 \mathrm{mV}$ between the systems, confirming that NGR has a significant impact on $V_{\text {oc }}$.

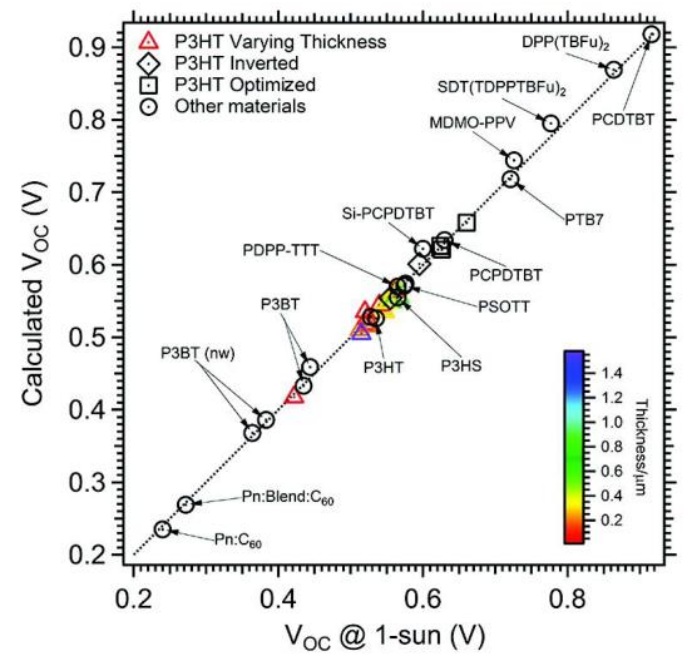

Figure 24. Predictions of 1 sun $V_{\mathrm{OC}}$ plotted against measured $V_{\mathrm{Oc}}$ under $100 \mathrm{~mW} \mathrm{~cm}^{-2}$ simulated AM1.5 illumination. The dashed line represents an exact 1:1 correlation, with average deviations from this line of approximately $\pm 5 \mathrm{mV}$. Reprinted with permission from ref. 77. Copyright 2012 American Chemical Society.

\section{Relevance to Fill Factor}

Vohra et al. have reported an efficient solar cell based on PNTz4T and $\mathrm{PC}_{71} \mathrm{BM}$ which exhibits a high FF of 0.73 and a PCE of $10.1 \%$ with 290 nm-thick active layer. ${ }^{6}$ From grazing-incidence wide-angle $X$-ray diffraction (GIWAXD) measurements, they revealed that PNTz4T forms a highly crystalline structure with face-on orientation, which is desirable for vertical charge transport in OPVs. Interestingly, PNTZ4T is more likely to form face-on structures in an inverted device structure with $\mathrm{ZnO}$ as a bottom electrode than in a conventional device structure with PEDOT:PSS, which they attribute to the 
difference of wettability of bottom electrodes. They also found that the face-on to edge-on ratio increased gradually with increasing active layer thickness, suggesting that edge-on crystallites are abundant at the film/substrate interface and that face-on crystallites are abundant in the bulk and the film/air interface. These unique characteristics for NTz-based polymer PNTz4T in the backbone orientation would facilitate the charge transport and improve FF. Kawashima et al. further modified NTz-based polymers through fluorine atom substitution. ${ }^{117}$ They synthesized PNTz4TF2 and PNTz4TF4 by introducing two and four fluorine atoms on the bithiophene moiety of PNTz4T, respectively. When blended with $\mathrm{PC}_{71} \mathrm{BM}, \mathrm{PNTz} 4 \mathrm{TF} 4$-based devices exhibited a degraded PCE of $\sim 6.5 \%$ whereas PNTz4TF2-based devices exhibited as high as 10.5\% PCE, which is slightly but statistically meaningfully higher than that of PNTz4T. A main contribution to the superior PCE for the PNTz4TF2based device is due to deeper HOMO energy of PNTz4TF2 owing to the fluorine substitution, resulting in a higher $V_{O C}$ of $0.82 \mathrm{~V}$ compared to $0.71 \mathrm{~V}$ for PNTZ4T-based devices. It is noteworthy that PNTZ4TF4based devices exhibit the highest $V_{\text {Oc }}$ of $0.93 \mathrm{~V}$. However, because of poor charge generation yield, the PCE of the device was degraded compared to PNTz4T-based device. In contrast, as shown in Figure $25 \mathrm{a}, \mathrm{FF}$ of the fluorinated devices was degraded to 0.67 for PNTZ4TF2

(a)

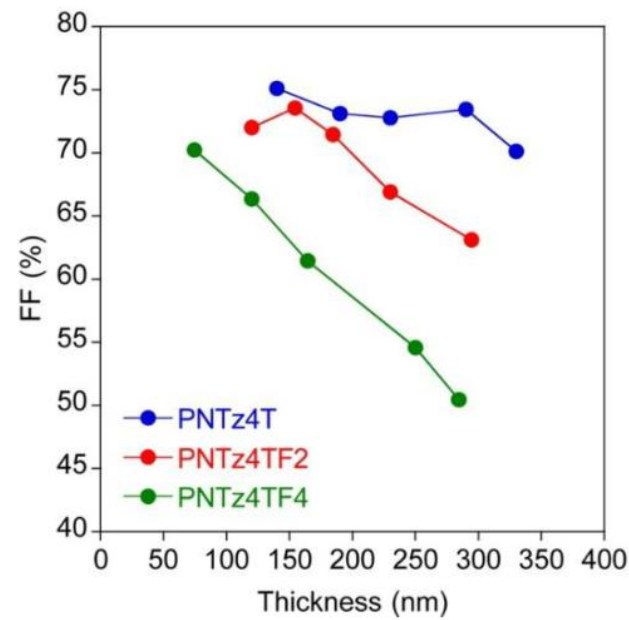

(b)

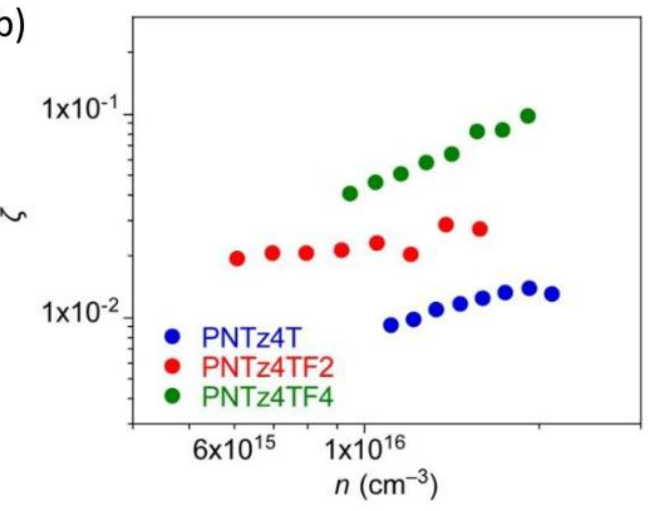

Figure 25. (a) Thickness dependence of FF of the solar cells based on PNTZ4T, PNTz4TF2, and PNTz4TF4 blended with $\mathrm{PC}_{71} \mathrm{BM}$. (b) Log-log plots of Langevin reduction factor against charge carrier density. Reproduced with permission from ref. 117. Copyright 2016 American Chemical Society. and 0.66 for PNTz4TF4. To explore the origin of these FFs, they conducted $J-V$ analysis using Equation 52 . Here, $\tau_{n}$ was evaluated by TPV/TPC and $n(V)$ was assumed to increase exponentially with an applied voltage. Based on these analyses, experimental $J-V$ curves were well reproduced, suggesting that NGR is a limiting factor of the FFs. As shown in Figure 25b, the reduction factor draws near unity, i.e. the Langevin limit, with increasing fluorine atoms. This is probably due to undesirable polymer crystallinity and backbone orientation. This study shows the importance, and at the same time difficulty, of precise molecular design to accomplish a high $V_{O C}$ and high FF simultaneously.

Neher et al. recently proposed a parameter $\vartheta$, which strongly correlates with $\mathrm{FF}$, by quantifying the competition between charge extraction and recombination. ${ }^{200,201}$

$$
\theta=\frac{\gamma G d^{4}}{\mu_{\mathrm{e}} \mu_{\mathrm{h}} V_{\text {int }}^{2}} \propto \frac{J_{\text {rec }}}{J_{\text {extr }}}
$$

where $V_{\text {int }}$ is an internal voltage and $J_{\text {rec }}$ and $J_{\text {extr }}$ are recombination and extraction current density, respectively. They confirmed that $\vartheta$ is a good measure of FF based on experimental results of solar cells of 15 different blend systems and 1D drift-diffusion simulation with parameters entering $\vartheta$ being varied over a wide range. They also proposed that the classical Shockley equation, which assumes high electrical conductivity, cannot be applied to OPVs, and obtained a new analytical expression for $J-V$ curves. ${ }^{202}$ The model is based on the fact that the low mobility of organic materials cause charge accumulation in the active layer leading to significant difference between the separation of the quasi Fermi levels (internal voltage, $\left.V_{\text {int }}\right)$ and the external voltage $\left(V_{\text {ext }}\right)$.

$$
\begin{gathered}
J=J_{0}\left\{\exp \left(\frac{q V_{\text {int }}}{k_{\mathrm{B}} T}\right)-1\right\}-J_{\text {gen }} \\
J_{0}=q d \gamma n_{\mathrm{i}}^{2} \\
V_{\text {ext }}=V_{\text {int }}+\frac{d}{\sigma} J
\end{gathered}
$$

where $n_{\mathrm{i}}$ is the intrinsic charge density and $\sigma$ is the electrical conductivity given by

$$
\sigma=2 q \sqrt{\mu_{\mathrm{e}} \mu_{\mathrm{h}}} n_{\mathrm{i}} \exp \left(\frac{q V_{\mathrm{int}}}{2 k_{\mathrm{B}} T}\right)
$$

Note that they assumed that the gradient of the quasi Fermi levels of electrons and holes are identical and constant throughout the active layer. The analytical equations were well consistent with the simulated $J-V$ curves. On the basis of these considerations, they obtained a new empirical equation of FF. ${ }^{201}$ A commonly used equation of $\mathrm{FF}$ is

$$
\mathrm{FF}=\frac{v_{\mathrm{OC}}-\ln \left(1+v_{\mathrm{OC}}\right)}{v_{\mathrm{OC}}+1}
$$

where $v_{O C}$ is the normalised open-circuit voltage defined as

$$
v_{\mathrm{OC}}=\frac{q V_{\mathrm{OC}}}{n_{\mathrm{id}} k_{\mathrm{B}} T}
$$

On the other hand, they proposed a new equation 


$$
\mathrm{FF}=\frac{v_{\mathrm{OC}}-\ln \left(0.79+0.66 v_{\mathrm{OC}}^{1.2}\right)}{v_{\mathrm{OC}}+1}
$$

where $v_{\mathrm{OC}}$ is expressed as

$$
v_{\mathrm{OC}}=\frac{q V_{\mathrm{OC}}}{(1+\alpha) k_{\mathrm{B}} T}
$$

Here, $\alpha$ is a figure of merit of charge collection efficiency, which is related to $\vartheta$ as follows:

$$
\alpha^{2}=\frac{q^{2} \gamma G d^{4}}{4 \mu_{\mathrm{e}} \mu_{\mathrm{h}}\left(k_{\mathrm{B}} T\right)^{2}}=\left(\frac{q V_{\mathrm{int}}}{2 k_{\mathrm{B}} T}\right)^{2} \theta=\left(\frac{V_{\mathrm{int}}}{2 V_{\mathrm{t}}}\right)^{2} \theta
$$

where $V_{\mathrm{t}}$ is the thermal voltage defined as $k_{\mathrm{B}} T / q$. Note that this $\alpha$ is different from the previous ones. Calculated FFs by Equations 63 and 65 are shown as broken and solid lines, respectively, in Figure 26. It shows that the new empirical equation coincides the simulated FF over a wider range of $\alpha$ compared to the conventional equation. The dependence of FF on $\alpha$ suggests that reducing NGR rate constant while realizing high electron and hole mobility directly leads to smaller $\alpha$ and therefore higher FF.

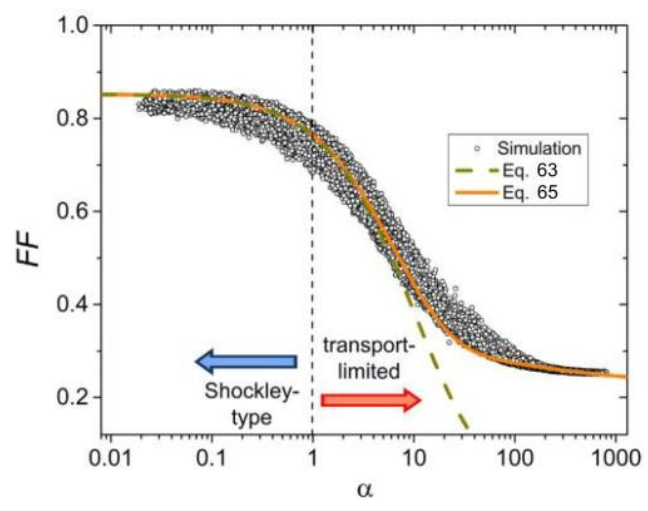

Figure 26. FF as a function of the figure of merit $\alpha$. Open circles represent $\mathrm{FF}$ of simulated $J-V$ curves with balanced mobilities and $V_{\mathrm{OC}}$ between 0.7 and $0.9 \mathrm{~V}$ (see ref. 200 for the simulation parameters). The broken and solid lines represent analytical dependence of FF on $\alpha$ according to Equations 63 and 65, respectively, with the normalised open-circuit voltage $V_{O C}$ expressed as Equation $66\left(T=300 \mathrm{~K}, V_{\mathrm{OC}}=0.8 \mathrm{~V}\right)$. Photocurrents will become strongly transport-limited for $\alpha>1$, resulting in a progressive decrease in FF when $\alpha>1$. Reprinted with permission from ref. 201. Copyright 2016 Springer Nature.

\section{Relevance to Voltage Loss}

Since the quasi-Fermi levels for electrons and holes are flat under an open-circuit condition, charge densities can be described as follows.

$$
\begin{aligned}
& n=N_{0} \exp \left(\frac{E_{\mathrm{fn}}-E_{\text {LUMO }, \mathrm{A}}}{k_{\mathrm{B}} T}\right) \\
& p=N_{0} \exp \left(\frac{E_{\text {HOMO,D }}-E_{\mathrm{fp}}}{k_{\mathrm{B}} T}\right)
\end{aligned}
$$

Here, $N_{0}$ is the effective density of states, typically $\sim 10^{21} \mathrm{~cm}^{-3}$ for organic semiconductors, $E_{\mathrm{fn}}$ and $E_{\mathrm{fp}}$ are the quasi-Fermi levels for electrons and holes, respectively, and $E_{\mathrm{LUMO}, \mathrm{A}}$ and $E_{\mathrm{HOMO}, \mathrm{D}}$ are the LUMO energy for an acceptor and the HOMO energy for a donor, respectively. Note that we assumed the same effective density of states for donor and acceptor for simplicity because it is not essential here. Under the open-circuit condition, the recombination rate $R$ expressed by Equation 1 equals to the generation rate $G$. The $V_{O C}$ is equal to the quasi-Fermi level splitting between electrons and holes as

$$
q V_{\mathrm{oC}}=E_{\mathrm{fn}}-E_{\mathrm{fp}}=E_{0}-k_{\mathrm{B}} T \ln \left(\frac{\gamma N_{0}^{2}}{G}\right)
$$

where $E_{0}$ represents the difference between $E_{\mathrm{LUMO}, \mathrm{A}}$ and $E_{\mathrm{HOMO}, \mathrm{D}}$. NGR via trap states originating from energetic disorders causes additional voltage loss. Considering the exponential distribution of tail states and recombination between trapped and free charges, Equation 70 turns into

$$
q V_{\mathrm{OC}}=E_{0}-n_{\mathrm{id}} k_{\mathrm{B}} T \ln \left(\frac{\gamma N_{\mathrm{t}} N}{G}\right)
$$

where $N_{\mathrm{t}}$ and $N$ are the total density of states of trapped and free charges, respectively. ${ }^{203}$ Since the ideality factor $n_{\text {id }}$ is related to the slope of the tail states as Equation 71, a larger degree of disorder leads to larger $n_{\text {id }}$ and therefore causes voltage loss.

To be more precise, $E_{0}$ should be replaced by the CTS energy $E_{\mathrm{CT}}$ to take into account Coulomb binding energy at the interface as below.

$$
q V_{\mathrm{OC}}=E_{\mathrm{CT}}-k_{\mathrm{B}} T \ln \left(\frac{q \mu \zeta N_{0}^{2}}{\varepsilon_{0} \varepsilon_{\mathrm{r}} G}\right)
$$

Equation 72 clearly indicates a strong correlation between $V_{\mathrm{OC}}$ and the reduction of NGR. The more the recombination is suppressed, the smaller the logarithmic term becomes, and the smaller the voltage loss will be.

Taking into account the equilibrium between CTS and FC as mentioned earlier, Burke et al., proposed the expression for $V_{O C}$ using parameters related to CTS

$$
q V_{\mathrm{OC}}=E_{\mathrm{CT}}-\frac{\sigma_{\mathrm{CT}}^{2}}{2 k_{\mathrm{B}} T}-k_{\mathrm{B}} T \ln \left(\frac{q f N_{0} L}{\tau_{\mathrm{CT}} J_{\mathrm{SC}}}\right)
$$

where $f, L$, and $\tau_{C T}$ are the volume fraction of the solar cell, active layer thickness, and lifetime of CTS, respectively. ${ }^{162}$ The CTS lifetime is typically in between $0.1 \mathrm{~ns}$ and $1 \mathrm{~ns}$, and rarely longer than $10 \mathrm{~ns}$. This is the reason why the difference between $E_{\mathrm{CT}}$ and $q V_{\mathrm{OC}}$ is almost always $0.5-0.7 \mathrm{eV}$ in most OPVs. They estimated that we would have an opportunity to obtain $120 \mathrm{mV}$ improvement in $V_{\mathrm{OC}}$ if we could increase CTS lifetime from 0.1 to $10 \mathrm{~ns}$.

The third term in Equation 73 includes radiative and nonradiative recombination losses because $k_{\mathrm{rec}}$ is given by $1 / \tau_{\mathrm{CT}}=k_{\mathrm{r}}+k_{\mathrm{nr}}$ where $k_{\mathrm{r}}$ and $k_{\mathrm{nr}}$ are radiative and nonradiative transition rate constant, respectively. In other words, the smaller $k_{\text {rec }}$ will give the smaller radiative and nonradiative recombination losses. Detailed balance analysis tells us that any type of solar cells must have unavoidable, intrinsic voltage loss relative to radiative recombination even though there are no disorders or trap sites. In the Shockley-Queisser (SQ) framework, the radiative recombination loss puts the upper limit of 
maximum achievable $q V_{\text {oc }}$ to be $\sim 0.3 \mathrm{eV}$ lower than the $E_{\mathrm{CT}}$. However, it is well known that the voltage loss in OPVs is much larger than the SQ limit. The difference between $E_{\mathrm{CT}}$ and $q V_{\mathrm{OC}}$ is typically $0.5-0.7 \mathrm{eV}$ as mentioned above ${ }^{21}$. This implies that there is another loss channel due to nonradiative recombination. The amount of voltage loss due to nonradiative recombination is quantified as

$$
\Delta V_{\mathrm{nr}}=-\frac{k_{\mathrm{B}} T}{q} \ln \left(\mathrm{EQE}_{\mathrm{EL}}\right) \sim-\frac{k_{\mathrm{B}} T}{q} \ln \left(\frac{p_{\mathrm{e}} k_{\mathrm{r}}}{p_{\mathrm{e}} k_{\mathrm{r}}+k_{\mathrm{nr}}}\right)
$$

where $E Q E_{E L}$ represents external quantum efficiency of EL from the solar cell device, $p_{\mathrm{e}}$ is an emission probability, and $k_{\mathrm{r}}\left(k_{\mathrm{nr}}\right)$ is (non-)radiative transition rate constant. ${ }^{204}$ Because the transition dipole moment of CTS is usually quite small due to poor spatial overlap between the donor HOMO and acceptor LUMO, $k_{n r}$ is much larger than $k_{\mathrm{r}}$ and hence $\mathrm{EQE}_{\mathrm{EL}}$ is reported typically in the order of $10^{-6}-10^{-7}$, resulting in additional $350-400 \mathrm{mV}$ voltage loss.

On the other hand, Benduhn et al. have found an inverse relationship between the $E_{\mathrm{CT}}$ and $\Delta V_{\mathrm{nr}}$ for a wide variety of fullerene-based solar cells as shown in Figure 27, which can be attributed to the energy gap law, or the transition rate in the Marcus inverted regime, for nonradiative transition of the CTS. ${ }^{20}$ In this regime, the nonradiative transition rate from the CTS with the lowest vibrational energy to an iso-energetic high vibrational state in the GS is proportional to the vibrational wave function overlap between those states, which decreases with increasing difference in vibrational quantum number. They tried to suppress nonradiative transition by replacing hydrogen in the $\mathrm{C}-\mathrm{H}$ bonds with deuterium. The results were, however, unpleasant for them. Deuteration had negligible impact on the photovoltaic performance and the EL spectra. They suggested that the large nonradiative recombination loss is unavoidable as it is intrinsic to the $\mathrm{C}-\mathrm{C}$ bond vibration.

On the other hand, one possibility for reducing nonradiative loss would be utilizing NFAs. Some recent studies have shown much higher $\mathrm{EQE}_{\mathrm{EL}} .{ }^{17,205-210}$ If we could obtain NFA-based devices with $\mathrm{EQE}_{\mathrm{EL}}$ two orders of magnitude larger $\left(\mathrm{EQE}_{\mathrm{EL}} \sim 10^{-4}\right)$ than conventional fullerene-based devices, the nonradiative loss would be $<250 \mathrm{mV}$. Such a high EQE $E_{\mathrm{EL}}$ has already been achieved for NFA-based OPVs where nonradiative loss was suppressed to only 200 meV. ${ }^{208,210}$ Recent studies suggest that radiative recombination rate for CTS in NFA-based OPVs could be enhanced because of intensity borrowing from a highly emissive local excitation (LE) state of NFA. ${ }^{211}$ Such a hybridisation between LE and CT states have been already proposed for fullerene-based OPVs with a small energy difference between LE and CT states. ${ }^{212,213}$ Indeed, PNOz4T/PC ${ }_{71} \mathrm{BM}$ polymer solar cells have been reported to exhibit a high EQE $E_{\mathrm{EL}}\left(\sim 10^{-4}\right)$ and hence a small nonradiative loss $(0.24 \mathrm{~V}) .{ }^{214}$ Thus, it would be a good strategy for reducing nonradiative losses to decrease the energy difference between LE and CT states. In particular, this effect would be larger in NFA-based OPVs than in fullerene-based OPVs because of much higher PL quantum efficiencies. As a result, nonradiative recombination loss would be relatively reduced. Nonetheless, this issue is still not fully understood. Thus, further studies should be needed.

To obtain highly emissive NFA materials, we need molecular design rules to suppress nonradiative transition rate. Recently, Sato et al. have theoretically calculated the nonradiative transition rate of

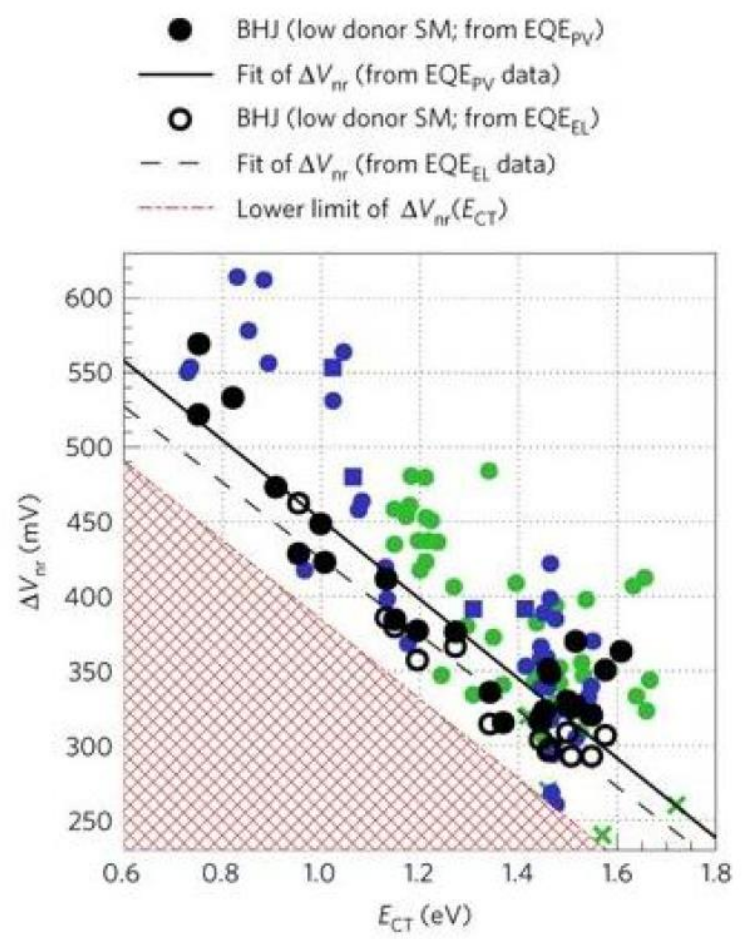

Figure 27. Nonradiative voltage loss $\Delta V_{\mathrm{nr}}$ plotted against the $E_{\mathrm{CT}}$. The filled symbols represent data calculated from EQE $E_{\mathrm{PV}}$ and the open symbols correspond to data measured by $\mathrm{EQE}_{\mathrm{EL}}$. The red dashed line shows an empirical lower limit of $\Delta V_{n r}$ as a function of the $E_{\text {CT. }}$. The circles represent $\mathrm{BHJ}$ OPVs whereas the squares represent $\mathrm{PHJ}$ devices. The black symbols represent a specific series of devices for which small molecule donors are diluted at $6 \mathrm{~mol} \%$ in $\mathrm{C}_{60}$ molecules, the blue symbols indicate small molecule donors at a usual donor/acceptor blend ratio, and the green symbols represent devices comprising polymer donors mixed with fullerenes for which the sensitively measured $E_{\mathrm{CQ}} \mathrm{PV}$ spectra were reanalysed for this plot. The crosses represent recently published OPVs for which data were available. Reprinted with permission from ref. 20. Copyright 2017 Springer Nature.

organic materials on the basis of vibronic coupling and related concepts. ${ }^{215}$ They successfully explained the difference in fluorescence properties of OLED materials and proposed a design principle for fluorescent molecules. Such theoretical molecular design guidelines would also be useful for developments of OPV materials with reduced nonradiative recombination.

\section{Concluding Remarks and Perspective to Future Developments}

As summarised in this review, suppression of the NGR is undoubtedly the top priority for further improving the device performance of OPVs. Reduced NGR compared with diffusion-limited Langevin theory by 2-4 orders of magnitude was reported for some blend systems. Recent experimental results show that blends employing highly crystalline polymers or fluorinated SMNFAs, which have strong inter/intramolecular interaction, exhibit relatively small reduction 
factors. This suggests that domain purity due to crystallization or aggregation is related to NGR reduction in blend films. Several mechanisms have proposed for the reduced NGR. Among them, many researches have shed light on the importance of crystallinity/morphology near the interface for suppression of the NGR.

The cascade energy landscape near the interface can enhance charge dissociation and hence suppress NGR effectively. However, it is yet difficult to accurately evaluate the local structure of the cascade and the energy difference between pure and mixed phase in nanoscale $\mathrm{BHJ}$ blend films. The influence of such cascade characteristics on NGR rate and solar cell performance should be studied. In order to improve PCE further, we need material/device design strategy to optimise local morphology. One possibility for further optimisation of blend morphology would be utilising ternary blend system, where the cascade energy landscape is artificially constructed. On the other hand, pure domains provide the greater number of charge states and therefore enhance entropy effect in the free energy. Entropy effect decreases Coulomb attraction energy and would be effective for not only efficient charge generation but also reduced NGR.

Recent improvements in the PCE of OPVs mainly relay on developments of new NFA materials. Of particular importance is that NFA-based OPVs can suppress voltage losses. One possible explanation is that hybridisation between LE and CT states could enhance radiative recombination rate of CTS and hence reduce nonradiative recombination loss relatively. Although this is true for both fullerene-based and NFA-based OPVs, this strategy would be more effective for NFA-based OPVs because of their higher PL quantum efficiencies especially. Thus, it would be of importance to develop highly emissive NFA materials in order to reduce nonradiative recombination losses. 


\section{Appendix}

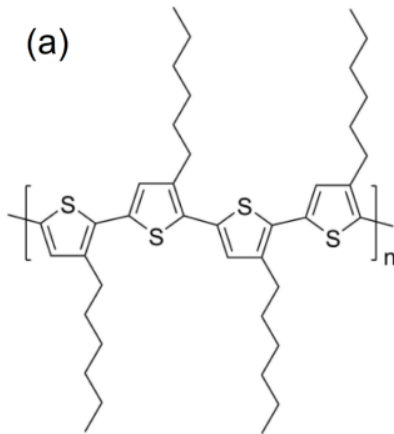

(d)

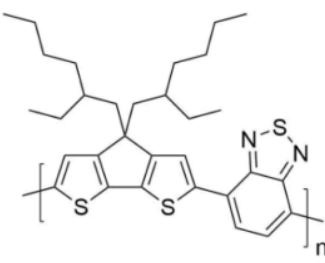

(b)

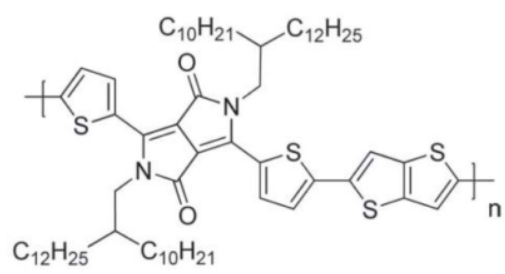

(e)<smiles>CCCCC(CC)COC</smiles>

(c)

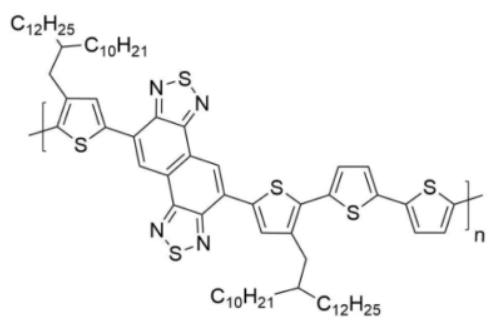

(f)

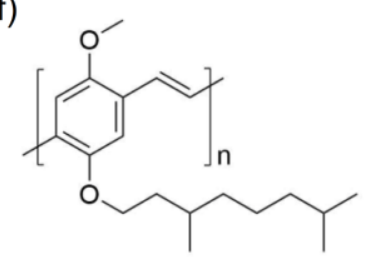

(g)

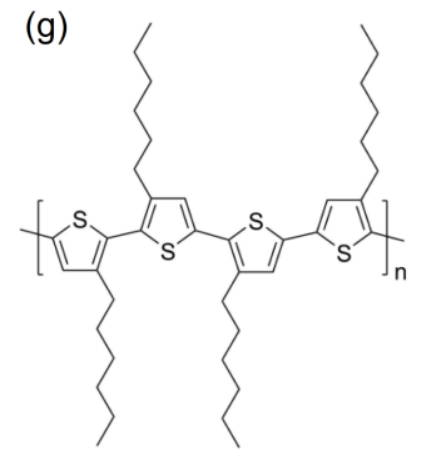

(h)

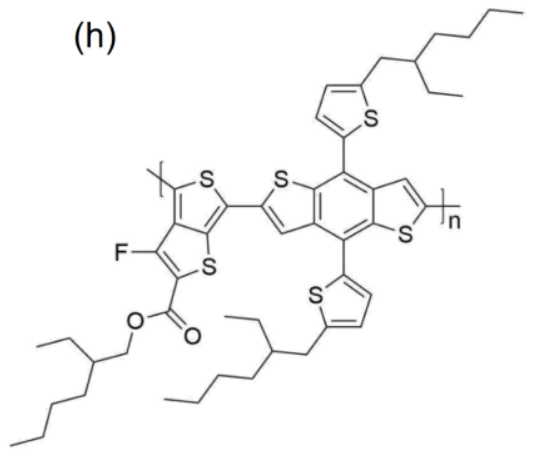

(i)

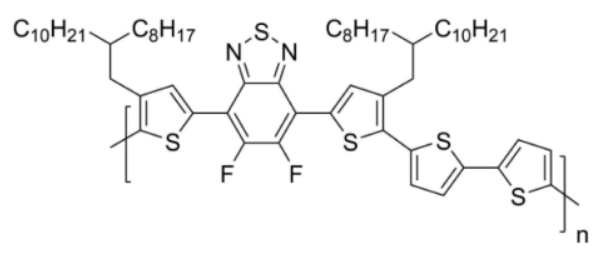

(j)

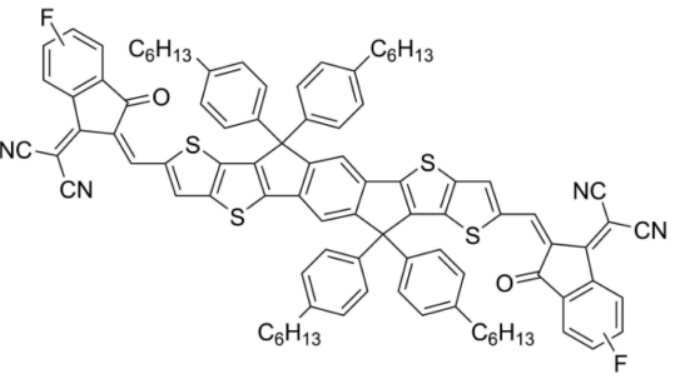

(I)

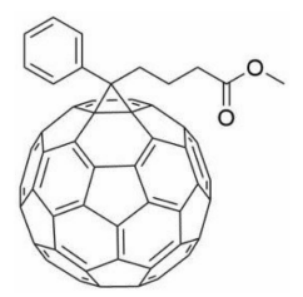

(m)

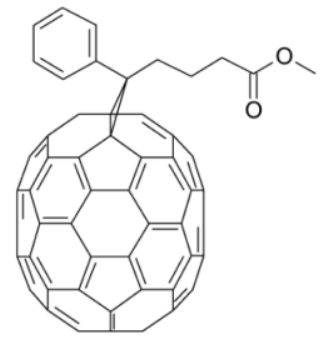

(k)

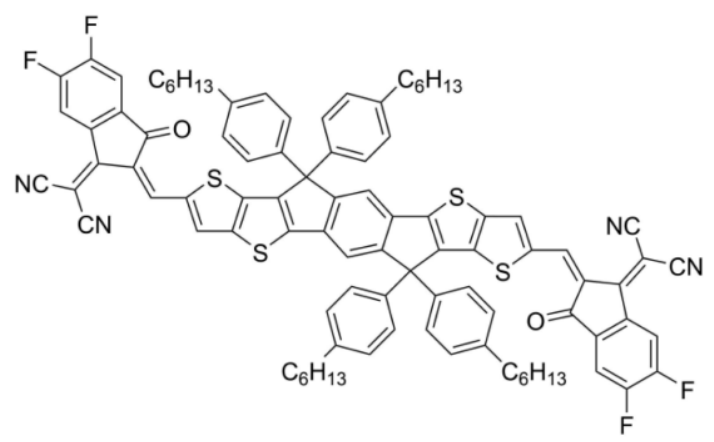

(n)

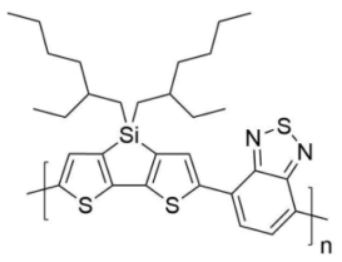

Figure A1. Chemical structures of (a) RR-P3HT, (b) DT-PDPP2T-TT, (c) PNTZ4T, (d) PCPDTBT, (e) PTB7, (f) MDMO-PPV, (g) RRa-P3HT, (h) PTB7-th, (i) PffBT4T-2OD, (j) ITIC-2F, (k) IT-4F, (I) PC 61 BM, (m) PC ${ }_{71} B M$, and (n) PSBTBT. 
(a)

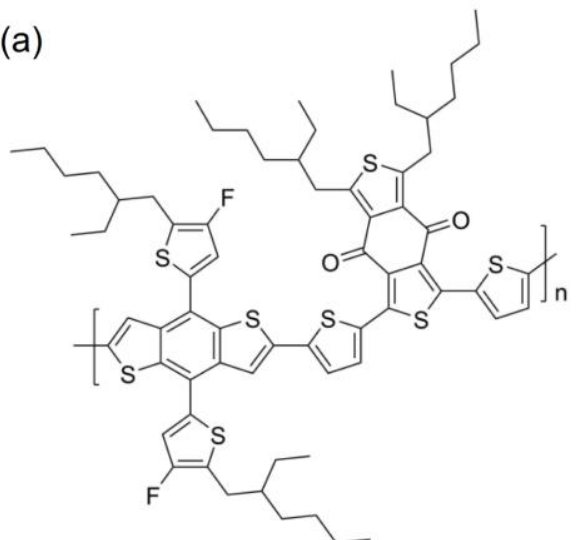

(c)

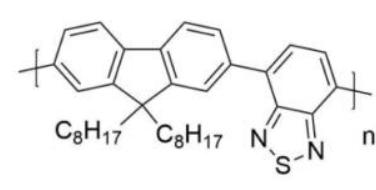

(f)

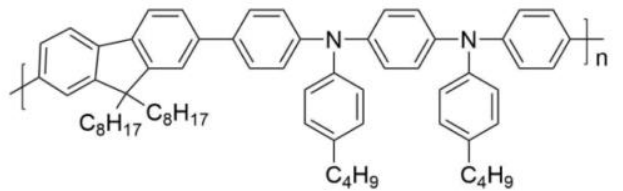

(g)
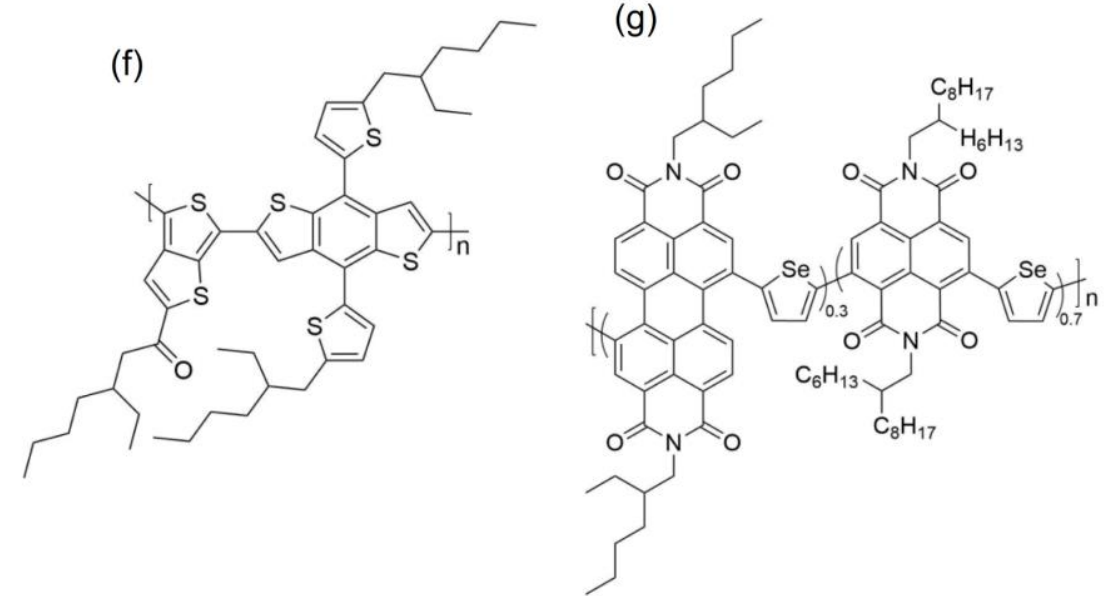

(b)

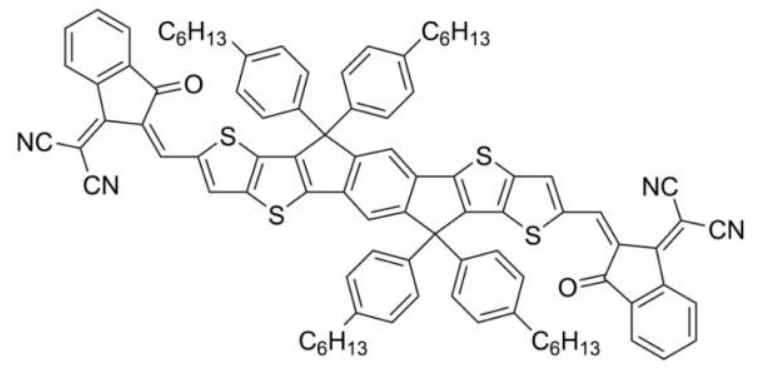

(e)

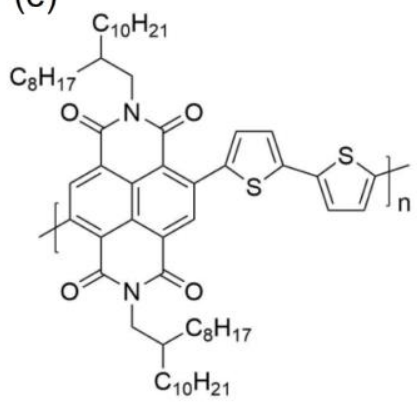

(i)

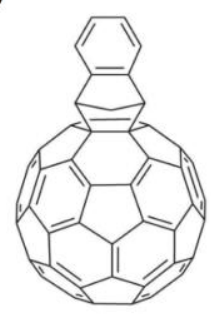

(j)

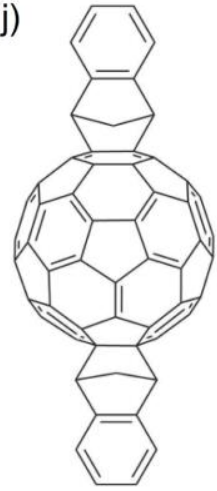

(k)

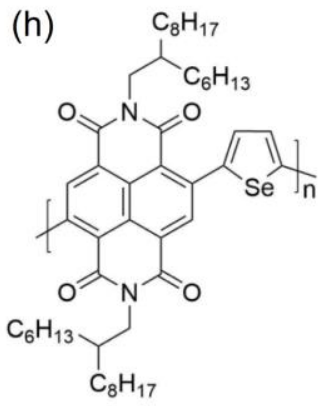

Figure A2. Chemical structures of (a) PBDBT-2F, (b) ITIC, (c) F8BT, (d) PFB, (e) N2200, (f) PBDTTT-CT, (g) 30PDI, (h) PNDIS-HD, (i)ICMA, (j) ICBA, and (k) PIPCP. 
(a)
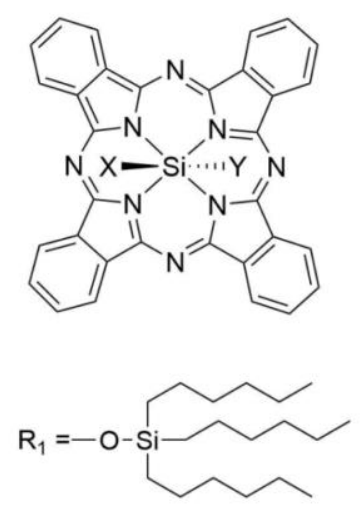

(b)

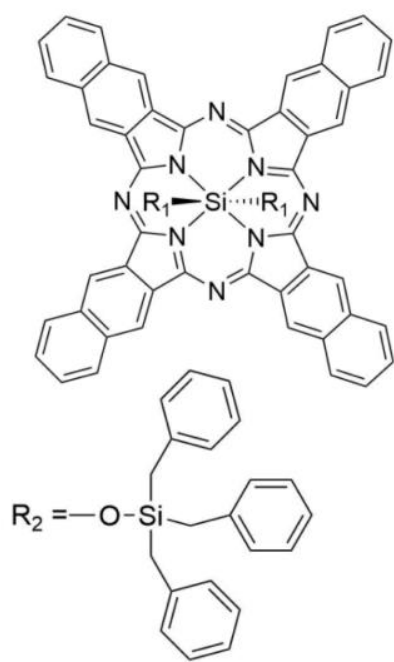

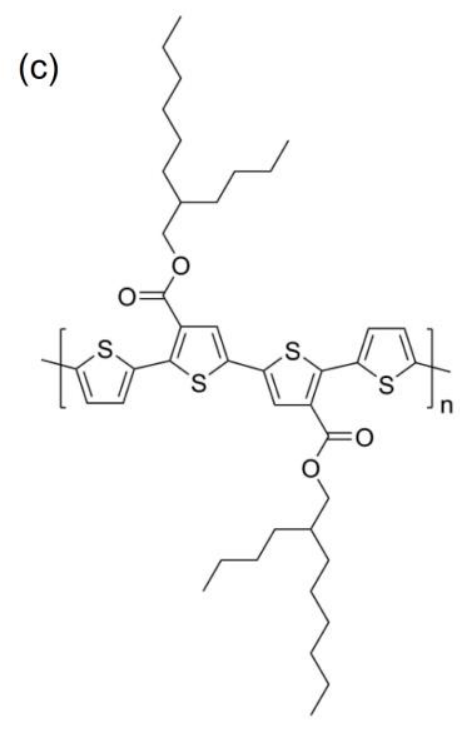

(d)

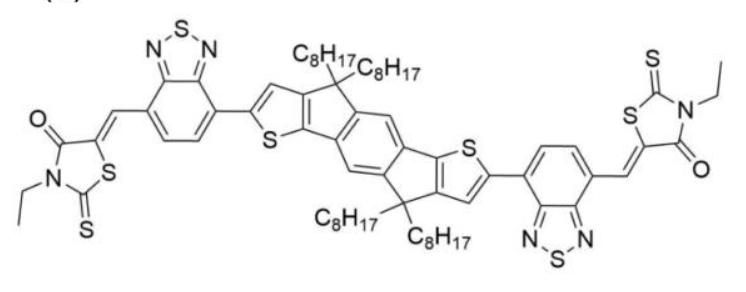

(e)

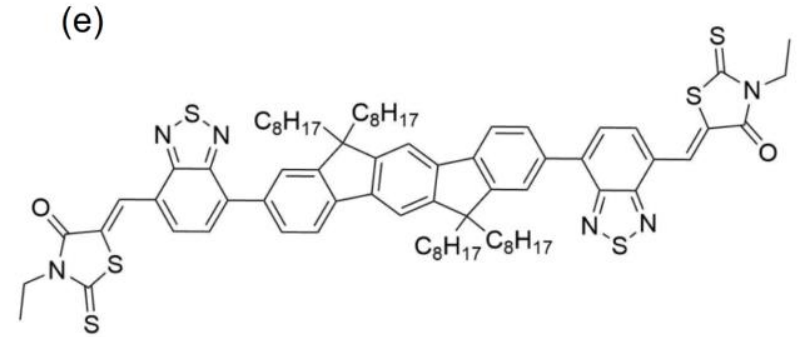

(f)

(g)

(h)
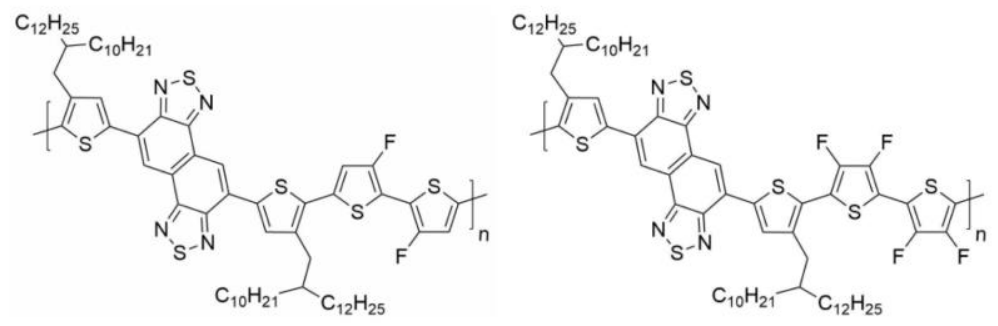

Figure A3. Chemical structures of (a) SiPc $\left(X=Y=R_{1}\right)$ or SiPcBz6 $\left(X=R_{1}, Y=R_{2}\right)$, (b) SiNc, (c) PDCBT, (d) IDTBR, (e) IDFBR, (f) PNTz4TF2, (g) PNTz4TF4, and (h) PNOz4T. 


\section{Conflicts of interest}

There are no conflicts to declare.

\section{Acknowledgements}

This work was partly supported by the JSPS KAKENHI Grant Number 17K14527, 18J20587, and 26248033, JST PRESTO program Grant Number JPMJPR1874, JST ALCA program Grant Number JPMJAL1404, and The Murata Science Foundation, Japan. Y.T. acknowledges financial support from Trycom Advance Co., Ltd. T.F. acknowledges support from the JSPS Research Fellowships for Young Scientists.

\section{Notes and references}

\section{Corresponding Authors}

*E-mail: tamai@photo.polym.kyoto-u.ac.jp

*E-mail: ohkita@photo.polym.kyoto-u.ac.jp

\section{ORCID}

Tomohiro Fukuhara: 0000-0002-2231-249X

Yasunari Tamai: 0000-0002-3074-0208

Hideo Ohkita: 0000-0002-7403-3492

\section{Biographies}

Tomohiro Fukuhara is a doctor course student of the Department of Polymer Chemistry at Kyoto University. He received his B.S. (2016) and M.S. (2018) degree from Kyoto University. His research focuses on charge recombination dynamics in organic photovoltaics.

Yasunari Tamai received his PhD from Kyoto University in 2013 on the excited state dynamics in nanostructured polymer systems. He was a postdoctoral fellow with Prof Sir Richard Friend at University of Cambridge, focussing on ultrafast spectroscopy for organic semiconductors. Since 2016, he has been an Assistant Professor at Kyoto University. Since 2018, he has also been a JST PRESTO researcher. His research interests include exciton and charge dynamics in organic nanomaterials. His current research focuses on photovoltaic conversion in organic systems.

Hideo Ohkita is a Professor in the Department of Polymer Chemistry at Kyoto University. He obtained a Doctoral degree in 1997 at Kyoto University. He became an Assistant Professor in 1997, was promoted to Associate Professor in 2006, and to Professor of the Department of Polymer Chemistry at Kyoto University in 2016. He concurrently worked as an academic visitor with Professor Durrant at Imperial College London from 2005 to 2006, and as a researcher in the Precursory Research for Embryonic Science and Technology (PRESTO) program "Photoenergy Conversion Systems and Materials for the Next Generation Solar Cells", Japan Science and Technology Agency (JST), from 2009 to 2015. His research interests include studying photophysics and photochemistry in polymer systems. His current research focuses on spectroscopic studies of photophysics in polymer solar cells.

\section{References}

1 S. Shaheen, C. Brabec, N. Sariciftci, F. Padinger, T. Fromherz and J. Hummelen, Appl. Phys. Lett., 2001, 78, 841.
2 F. Padinger, R. Rittberger and N. Sariciftci, Adv. Funct. Mater., 2003, 13, 85

3 G. Li, V. Shrotriya, J. Huang, Y. Yao, T. Moriarty, K. Emery and Y. Yang, Nat. Mater., 2005, 4, 864.

4 J. Peet, J. Y. Kim, N. E. Coates, W. L. Ma, D. Moses, A. J. Heeger and G. C. Bazan, Nat. Mater., 2007, 6, 497.

5 Y. Liu, J. Zhao, Z. Li, C. Mu, W. Ma, H. Hu, K. Jiang, H. Lin, H. Ade and H. Yan, Nat. Commun., 2014, 5, 5293.

6 V. Vohra, K. Kawashima, T. Kakara, T. Koganezawa, I. Osaka and K. Takimiya, Nat. Photonics, 2015, 9, 403.

7 J. Zhao, Y. Li, G. Yang, K. Jiang, H. Lin, H. Ade, W. Ma and H. Yan, Nat. Energy, 2016, 1, 15027.

8 J. Zhang, Y. Li, J. Huang, H. Hu, G. Zhang, T. Ma, P. C. Y. Chow, H. Ade, D. Pan and H. Yan, J. Am. Chem. Soc., 2017, 139, 16092.

9 S. Zhang, Y. Qin, J. Zhu and J. Hou, Adv. Mater., 2018, 30, 1800868

10 L. Zhan, S. Li, T. K. Lau, Y. Cui, X. Lu, M. Shi, C. Z. Li, H. Li, J. Hou and H. Chen, Energy Environ. Sci., 2020, 13, 635.

11 L. Liu, Y. Kan, K. Gao, J. Wang, M. Zhao, H. Chen, C. Zhao, T. Jiu, A. K. Y. Jen and Y. Li, Adv. Mater., 2020, 32, 1907604.

12 Y. Cui, H. Yao, J. Zhang, K. Xian, T. Zhang, L. Hong, Y. Wang, Y. Xu, K. Ma, C. An, C. He, Z. Wei, F. Gao and J. Hou, Adv. Mater., 2020, 32, 1908205

13 Q. Liu, Y. Jiang, K. Jin, J. Qin, J. Xu, W. Li, J. Xiong, J. Liu, Z. Xiao, K. Sun, S. Yang, X. Zhang, L. Ding, Sci. Bull., 2020, 65, 272.

14 X. Guo, N. Zhou, S. J. Lou, J. Smith, D. B. Tice, J. W. Hennek, R. Ponce Ortiz, J. T. Lopez Navarrete, S. Li, J. Strzalka, L. X. Chen, R. P. H. Chang, A. Facchetti and T. J. Marks, Nat. Photonics, 2013, 7, 825.

15 G. Dennler, M. C. Scharber, and C. J. Brabec, Adv. Mater., 2009, 21, 1323

16 K. Kawashima, Y. Tamai, H. Ohkita, I. Osaka and K. Takimiya, Nat. Commun., 2015, 6, 10085.

17 J. Liu, S. Chen, D. Qian, B. Gautam, G. Yang, J. Zhao, J. Bergqvist, F. Zhang, W. Ma and H. Ade, Nat. Energy, 2016, 1, 16089.

18 S. M. Menke, A. Sadhanala, M. Nikolka, N. A. Ran, M. K. Ravva, S. Abdel-Azeim, H. L. Stern, M. Wang, H. Sirringhaus, T.-Q. Nguyen, J. Brédas, G. C. Bazan and R. H. Friend, ACS Nano, 2016, 10, 10736.

19 N. A. Ran, J. A. Love, C. J. Takacs, A. Sadhanala, J. K. Beavers, S. D. Collins, Y. Huang, M. Wang, R. H. Friend, G. C. Bazan and T.-Q. Nguyen, Adv. Mater., 2016, 28, 1482. 
20 J. Benduhn, K. Tvingstedt, F. Piersimoni, S. Ullbrich, Y. Fan, M. Tropiano, K. A. McGarry, O. Zeika, M. K. Riede, C. J. Douglas, S. Barlow, S. R. Marder, D. Neher, D. Spoltore and K. Vandewal, Nat. Energy, 2017, 2, 17053.

21 S. M. Menke, N. A. Ran, G. C. Bazan and R. H. Friend, Joule, 2018 2, 25.

22 C. Wang, X. Xu, W. Zhang, J. Bergqvist, Y. Xia, X. Meng, K. Bini, W. Ma, A. Yartsev, K. Vandewal, M. R. Andersson, O. Inganäs, M. Fahlman and E. Wang, Adv. Energy Mater., 2016, 6, 1600148.

23 H. Zhang, S. Li, B. Xu, H. Yao, B. Yang and J. Hou, J. Mater. Chem. A, 2016, 4, 18043.

24 X. Xu, T. Yu, Z. Bi, W. Ma, Y. Li and Q. Peng, Adv. Mater., 2018, 30, 1703973.

25 G. Adriaenssens and V. Arkhipov, Solid State Commun., 1997, 103, 541.

26 A. Pivrikas, N. S. Sariciftci, G. Juŝka and R. Österbacka, Prog. Photovoltaics, 2007, 15, 677.

27 J. Szmytkowski, Chem. Phys. Lett., 2009, 470, 123.

28 C. Deibel, A. Wagenpfahl and V. Dyakonov, Phys. Rev. B, 2009, 80, 075203 .

29 M. Hilczer and M. Tachiya, J. Phys. Chem. C, 2010, 114, 6808.

30 R. Österbacka, A. Pivrikas, G. Juŝka, A. Poskus, H. Aarnio, G. Sliauzys, K. Genevicius, K. Arlauskas and N. S. Sariciftci, IEEE J. Sel. Top. Quantum Electron., 2010, 16, 1738.

31 C. M. Proctor, M. Kuik and T.-Q. Nguyen, Prog. Polym. Sci., 2013, 38, 1941.

32 D. H. K. Murthy, A. Melianas, Z. Tang, G. Juŝka, K. Arlauskas, F. Zhang, L. D. A. Siebbeles, O. Inganäs and T. J. Savenije, Adv. Funct. Mater., 2013, 23, 4262.

33 G. Lakhwani, A. Rao and R. H. Friend, Annu. Rev. Phys. Chem., 2014, 65, 557.

34 R. Tempelaar, L. J. A. Koster, R. W. A. Havenith, J. Knoester and T. L. C. Jansen, J. Phys. Chem. Lett., 2016, 7, 198.

35 A. Wagenpfahl, J. Phys. Condes. Matter, 2017, 29, 373001.

36 C. Goehler, A. Wagenpfahl and C. Deibel, Adv. Electron. Mater., 2018, 4, 1700505.

37 S. Shoaee, M. Stolterfoht and D. Neher, Adv. Energy Mater., 2018, 8, 1703355.
38 Y. Tamai, H. Ohkita, H. Benten and S. Ito, J. Phys. Chem. Lett., 2015, 6, 3417.

39 J. J. M. Halls, C. A. Walsh, N. C. Greenham, E. A. Marseglia, R. H. Friend, S. C. Moratti and A. B. Holmes, Nature, 1995, 376, 498.

40 G. Yu, J. Gao, J. C. Hummelen, F. Wudl and A. J. Heeger, Science, 1995, 270, 1789.

41 H. Ohkita, S. Cook, Y. Astuti, W. Duffy, S. Tierney, W. Zhang, M. Heeney, I. McCulloch, J. Nelson, D. D. C. Bradley and J. R. Durrant, J. Am. Chem. Soc., 2008, 130, 3030.

42 T. M. Clarke and J. R. Durrant, Chem. Rev., 2010, 110, 6736.

43 F. Gao and O. Inganäs, Phys. Chem. Chem. Phys., 2014, 16, 20291.

44 J. Guo, H. Ohkita, H. Benten and S. Ito, J. Am. Chem. Soc., 2010, 132,6154 .

45 F. Etzold, I. A. Howard, R. Mauer, M. Meister, T. Kim, K. Lee, N. S. Baek and F. Laquai, J. Am. Chem. Soc., 2011, 133, 9469.

46 S. Honda, S. Yokoya, H. Ohkita, H. Benten and S. Ito, J. Phys. Chem. C, 2011, 115, 11306.

47 Y. Tamai, K. Tsuda, H. Ohkita, H. Benten and S. Ito, Phys. Chem. Chem. Phys., 2014, 16, 20338.

48 S. Gélinas, A. Rao, A. Kumar, S. L. Smith, A. W. Chin, J. Clark, T. S. van der Poll, G. C. Bazan and R. H. Friend, Science, 2014, 343, 512.

49 A. C. Jakowetz, M. L. Bohm, J. Zhang, A. Sadhanala, S. Huettner, A. A. Bakulin, A. Rao and R. H. Friend, J. Am. Chem. Soc., 2016, 138, 11672.

50 A. C. Jakowetz, M. L. Bohm, A. Sadhanala, S. Huettner, A. Rao and R. H. Friend, Nat. Mater., 2017, 16, 551.

51 Y. Tamai, Y. Fan, V. O. Kim, K. Ziabrev, A. Rao, S. Barlow, S. R. Marder, R. H. Friend and S. M. Menke, ACS Nano, 2017, 11, 12473.

52 S. H. Park, A. Roy, S. Beaupre, S. Cho, N. Coates, J. S. Moon, D. Moses, M. Leclerc, K. Lee and A. J. Heeger, Nat. Photonics, 2009, 3, 297.

53 J. A. Bartelt, Z. M. Beiley, E. T. Hoke, W. R. Mateker, J. D. Douglas, B. A. Collins, J. R. Tumbleston, K. R. Graham, A. Amassian, H. Ade, J. M. J. Frechet, M. F. Toney and M. D. McGehee, Adv. Energy Mater., 2013, 3, 364.

54 X. Xu, Z. Li, W. Zhang, X. Meng, X. Zou, D. Di Carlo Rasi, W. Ma, A. Yartsev, M. R. Andersson, R. A. J. Janssen and E. Wang, Adv. Energy Mater., 2018, 8, 1700908.

55 R. Hall, Phys. Rev., 1952, 87, 387. 
56 W. Shockley and W. Read, Phys. Rev., 1952, 87, 835.

57 A. Richter, S. W. Glunz, F. Werner, J. Schmidt and A. Cuevas, Phys. Rev. B, 2012, 86, 165202.

58 T. Kirchartz, B. E. Pieters, J. Kirkpatrick, U. Rau and J. Nelson, Phys. Rev. B, 2011, 83, 115209.

59 T. Kirchartz and J. Nelson, Phys. Rev. B, 2012, 86, 165201.

60 P. Langevin, Ann. Chim. Phys., 1903, 28, 433.

61 M. Pope and C. E. Swenberg, Electronic Processes in Organic Crystals and Polymers, Oxford Scientific Publications, New York, 1999.

62 P. Debye, J. Electrochem. Soc., 1942, 82, 265.

63 M. V. Smoluchowski, Z. Phys. Chem., 1917, 92, 129.

64 M. M. Mandoc, W. Veurman, L. J. A. Koster, B. de Boer and P. W. M. Blom, Adv. Funct. Mater., 2007, 17, 2167.

65 C. R. McNeill, J. J. M. Halls, R. Wilson, G. L. Whiting, S. Berkebile, M. G. Ramsey, R. H. Friend and N. C. Greenham, Adv. Funct. Mater., 2008, 18, 2309.

66 S. R. Cowan, A. Roy and A. J. Heeger, Phys. Rev. B, 2010, 82, 245207.

67 L. Lu, W. Chen, T. Xu and L. Yu, Nat. Commun., 2015, 6, 7327.

68 L. J. A. Koster, M. Kemerink, M. M. Wienk, K. Maturova and R. A. J. Janssen, Adv. Mater., 2011, 23, 1670.

69 C. G. Shuttle, B. O'Regan, A. M. Ballantyne, J. Nelson, D. D. C. Bradley, J. de Mello and J. R. Durrant, Appl. Phys. Lett., 2008, 92, 093311.

70 A. Foertig, A. Baumann, D. Rauh, V. Dyakonov and C. Deibel, Appl. Phys. Lett., 2009, 95, 052104.

71 A. Maurano, R. Hamilton, C. G. Shuttle, A. M. Ballantyne, J. Nelson, B. O'Regan, W. Zhang, I. McCulloch, H. Azimi, M. Morana, C. J. Brabec and J. R. Durrant, Adv. Mater., 2010, 22, 4987.

72 C. G. Shuttle, R. Hamilton, B. C. O'Regan, J. Nelson and J. R. Durrant, Proc. Natl. Acad. Sci. U. S. A., 2010, 107, 16448.

73 Z. Li, F. Gao, N. C. Greenham and C. R. McNeill, Adv. Funct. Mater., 2011, 21, 1419.

74 A. Maurano, C. C. Shuttle, R. Hamilton, A. M. Ballantyne, J. Nelson, W. Zhang, M. Heeney and J. R. Durrant, J. Phys. Chem. $C$, 2011, 115, 5947.
75 A. Foertig, J. Rauh, V. Dyakonov and C. Deibel, Phys. Rev. B, 2012, 86,115302 .

76 D. Credgington, F. C. Jamieson, B. Walker, T.-Q. Nguyen and J. R. Durrant, Adv. Mater., 2012, 24, 2135.

77 D. Credgington and J. R. Durrant, J. Phys. Chem. Lett., 2012, 3, 1465.

78 D. Spoltore, W. D. Oosterbaan, S. Khelifi, J. N. Clifford, A. Viterisi, E. Palomares, M. Burgelman, L. Lutsen, D. Vanderzande and J. Manca, Adv. Energy Mater., 2013, 3, 466.

79 S. Khelifi, E. Voroshazi, D. Spoltore, F. Piersimoni, S. Bertho, T. Aernouts, J. Manca, J. Lauwaert, H. Vrielinck and M. Burgelman, Sol. Energy Mater. Sol. Cells, 2014, 120, 244.

80 C. G. Shuttle, A. Maurano, R. Hamilton, B. O'Regan, J. C. de Mello and J. R. Durrant, Appl. Phys. Lett., 2008, 93, 183501.

81 G. F. A. Dibb, F. C. Jamieson, A. Maurano, J. Nelson and J. R. Durrant, J. Phys. Chem. Lett., 2013, 4, 803.

82 B. Wright, Y. Nakajima, T. M. Clarke, K. Okuda, H. Paananen, A. J. Mozer and S. Mori, Adv. Energy Mater., 2017, 7, 1602026.

83 T. M. Clarke, J. Peet, P. Denk, G. Dennler, C. Lungenschmied and A. J. Mozer, Energy Environ. Sci., 2012, 5, 5241.

84 T. M. Clarke, C. Lungenschmied, J. Peet, N. Drolet, K. Sunahara, A. Furube and A. J. Mozer, Adv. Energy Mater., 2013, 3, 1473.

85 G. Zhang, T. M. Clarke and A. J. Mozer, J. Phys. Chem. C, 2016, 120, 7033 .

86 C. G. Shuttle, B. O'Regan, A. M. Ballantyne, J. Nelson, D. D. C. Bradley and J. R. Durrant, Phys. Rev. B, 2008, 78, 113201.

87 J. Guo, H. Ohkita, S. Yokoya, H. Benten and S. Ito, J. Am. Chem Soc., 2010, 132, 9631.

88 H. Ohkita and S. Ito, Polymer, 2011, 52, 4397.

89 Y. Tamai, H. Ohkita, H. Benten and S. Ito, J. Phys. Chem. C, 2013, 117, 10277.

90 H. Ohkita and S. Ito, "Exciton and Charge Dynamics in Polymer Solar Cells Studied by Transient Absorption Spectroscopy", in Organic Solar Cells, ed. W. H. Choy, Springer-Verlag, London, 2013 pp. 103-137.

91 Y. Tamai, Y. Matsuura, H. Ohkita, H. Benten and S. Ito, J. Phys. Chem. Lett., 2014, 5, 399.

92 Y. Tamai, H. Ohkita, H. Benten and S. Ito, Chem. Mater., 2014, 26, 2733. 
93 H. Ohkita, Y. Tamai, H. Benten and S. Ito, IEEE J. Sel. Top. Quantum Electron., 2016, 22, 4100112.

94 G. Juŝka, K. Arlauskas, M. Viliunas and J. Kocka, Phys. Rev. Lett., 2000, 84, 4946

95 G. Juŝka, K. Arlauskas, M. Viliunas, K. Genevicius, R. Österbacka and H. Stubb, Phys. Rev. B, 2000, 62, R16235.

96 A. Mozer, G. Dennler, N. Sariciftci, M. Westerling, A. Pivrikas, R. Österbacka and G. Juŝka, Phys. Rev. B, 2005, 72, 035217.

97 A. Mozer, N. Sariciftci, L. Lutsen, D. Vanderzande, R. Österbacka, M. Westerling and G. Juŝka, Appl. Phys. Lett., 2005, 86, 112104.

98 G. Dennler, A. Mozer, G. Juŝka, A. Pivrikas, R. Österbacka, A. Fuchsbauer and N. Sariciftci, Org. Electron., 2006, 7, 229.

99 C. Deibel, Phys. Status Solidi A-Appl. Mater., 2009, 206, 2731.

100 J. Lorrmann, B. H. Badada, O. Inganäs, V. Dyakonov and C. Deibel, J. Appl. Phys., 2010, 108, 113705.

101 A. Armin, G. Juŝka, M. Ullah, M. Velusamy, P. L. Burn, P. Meredith and A. Pivrikas, Adv. Energy Mater., 2014, 4, 1300954.

102 J. Peng, X. Chen, Y. Chen, O. J. Sandberg, R. Österbacka and Z. Liang, Adv. Electron. Mater., 2016, 2, 1500333.

103 J. Vazgela, K. Genevicius and G. Juŝka, Chem. Phys., 2016, 478, 126.

104 O. J. Sandberg, M. Nyman, S. Dahlstrom, S. Sanden, B. Torngren, J. Smatt and R. Österbacka, Appl. Phys. Lett., 2017, 110, 153504.

105 M. Stephen, K. Genevicius, G. Juŝka, K. Arlauskas and R. C. Hiorns, Polym. Int., 2017, 66, 13.

106 J. Kniepert, M. Schubert, J. C. Blakesley and D. Neher, J. Phys. Chem. Lett., 2011, 2, 700.

107 S. Albrecht, W. Schindler, J. Kurpiers, J. Kniepert, J. C. Blakesley, I. Dumsch, S. Allard, K. Fostiropoulos, U. Scherf and D. Neher, J. Phys. Chem. Lett., 2012, 3, 640.

108 S. Albrecht, J. R. Tumbleston, S. Janietz, I. Dumsch, S. Allard, U. Scherf, H. Ade and D. Neher, J. Phys. Chem. Lett., 2014, 5, 1131.

109 J. Kniepert, I. Lange, N. J. van der Kaap, L. J. A. Koster and D. Neher, Adv. Energy Mater., 2014, 4, 1301401.

110 G. J. A. H. Wetzelaer, N. J. V. Kaap, L. J. A. Koster and P. W. M. Blom, Adv. Energy Mater., 2013, 3, 1130.

111 L. M. Rosenberg, and M. A. Lampert, J. Appl. Phys., 1970, 41, 508.
112 T. M. Clarke, C. Lungenschmied, J. Peet, N. Drolet and A. J. Mozer, Adv. Energy Mater., 2015, 5, 1401345.

113 C. Deibel, A. Baumann and V. Dyakonov, Appl. Phys. Lett., 2008, 93, 163303.

114 R. Hamilton, C. G. Shuttle, B. O'Regan, T. C. Hammant, J. Nelson and J. R. Durrant, J. Phys. Chem. Lett., 2010, 1, 1432.

115 T. Fukuhara, M. Osaka, Y. Tamai, H. Ohkita, H. Benten and S. Ito, J. Photopolym. Sci. Technol., 2016, 29, 575.

116 T. Fukuhara, Y. Tamai, I. Osaka and H. Ohkita, Jpn. J. Appl. Phys., 2018, 57, 08RE01.

117 K. Kawashima, T. Fukuhara, Y. Suda, Y. Suzuki, T. Koganezawa, H. Yoshida, H. Ohkita, I. Osaka and K. Takimiya, J. Am. Chem. Soc., 2016, 138, 10265.

118 M. Nyman, O. J. Sandberg and R. Österbacka, Adv. Energy Mater., 2015, 5, 1400890.

119 A. Armin, Z. Chen, Y. Jin, K. Zhang, F. Huang and S. Shoaee, Adv. Energy Mater., 2018, 8, 1701450.

120 M. C. Heiber, T. Okubo, S. J. Ko, B. R. Luginbuhl, N. A. Ran, M. Wang, H. Wang, M. A. Uddin, H. Y. Woo, G. C. Bazan and T.-Q. Nguyen, Energy Environ. Sci., 2018, 11, 3019.

121 C. Vijila, S. P. Singh, E. Williams, P. Sonar, A. Pivrikas, B. Philippa, R. White, E. N. Kumar, S. G. Sandhya, S. Gorelik, J. Hobley, A. Furube, H. Matsuzaki and R. Katoh, J. Appl. Phys., 2013, 114, 184503.

122 H. Cha, S. Wheeler, S. Holliday, S. D. Dimitrov, A. Wadsworth, H. H. Lee, D. Baran, I. McCulloch and J. R. Durrant, Adv. Funct. Mater., 2018, 28, 1704389.

123 S. Albrecht, S. Janietz, W. Schindler, J. Frisch, J. Kurpiers, J. Kniepert, S. Inal, P. Pingel, K. Fostiropoulos, N. Koch and D. Neher, J. Am. Chem. Soc., 2012, 134, 14932.

124 T. M. Clarke, J. Peet, A. Nattestad, N. Drolet, G. Dennler, C. Lungenschmied, M. Leclerc and A. J. Mozer, Org. Electron., 2012, 13, 2639 .

125 G. Juŝka, K. Arlauskas, J. Stuchlik and R. Österbacka, J. Noncryst. Solids, 2006, 352, 1167.

126 X. Zhang, X. Zuo, S. Xie, J. Yuan, H. Zhou and Y. Zhang, J. Mater. Chem. A, 2017, 5, 17230.

127 X. Zhang, D. Zhang, Q. Zhou, R. Wang, J. Zhou, J. Wang, H. Zhou and Y. Zhang, Nano Energy, 2019, 56, 494.

128 S. M. Hosseini, S. Roland, J. Kurpiers, Z. Chen, K. Zhang, F. Huang, A. Armin, D. Neher and S. Shoaee, J. Phys. Chem. C, 2019, 123, 6823. 
129 J. Vollbrecht, V. V. Brus, S. J. Ko, J. Lee, A. Karki, D. X. Cao, K. Cho, G. C. Bazan and T.-Q. Nguyen, Adv. Energy Mater., 2019, 9, 1901438.

130 Y. Xie, W. Huang, Q. Liang, J. Zhu, Z. Cong, F. Lin, S. Yi, G. Luo T. Yang, S. Liu, Z. He, Y. Liang, X. Zhan, C. Gao, H. Wu and Y. Cao, ACS Energy Lett., 2019, 4, 8.

131 X. Song, N. Gasparini, M. M. Nahid, H. Chen, S. M. Macphee, W. Zhang, V. Norman, C. Zhu, D. Bryant, H. Ade, I. McCulloch and D. Baran, Adv. Funct. Mater., 2018, 28, 1802895

132 J. Wang, S. Xie, D. Zhang, R. Wang, Z. Zheng, H. Zhou and Y. Zhang, J. Mater. Chem. A, 2018, 6, 19934.

133 S. Roland, M. Schubert, B. A. Collins, J. Kurpiers, Z. Chen, A. Facchetti, H. Ade and D. Neher, J. Phys. Chem. Lett., 2014, 5, 2815.

134 A. Yin, D. Zhang, S. H. Cheung, S. K. So, Z. Fu, L. Ying, F. Huang, H. Zhou and Y. Zhang, J .Mater. Chem. C, 2018, 6, 7855.

135 S. Karuthedath, A. Melianas, Z. Kan, V. Pranculis, M. Wohlfahrt, J. I. Khan, J. Gorenflot, Y. Xia, O. Inganäs, V. Gulbinas, M. Kemerink and F. Laquai, J. Mater. Chem. A, 2018, 6, 7428

136 H. Choi, S. Ko, T. Kim, P. Morin, B. Walker, B. H. Lee, M. Leclerc, J. Y. Kim and A. J. Heeger, Adv. Mater., 2015, 27, 3318.

137 Z. Zheng, Q. Hu, S. Zhang, D. Zhang, J. Wang, S. Xie, R. Wang, Y. Qin, W. Li, L. Hong, N. Liang, F. Liu, Y. Zhang, Z. Wei, Z. Tang, T. P. Russell, J. Hou and H. Zhou, Adv. Mater., 2018, 30, 1801801.

138 W. Zhao, S. Li, H. Yao, S. Zhang, Y. Zhang, B. Yang and J. Hou, J. Am. Chem. Soc., 2017, 139, 7148.

139 W. Li, L. Ye, S. Li, H. Yao, H. Ade and J. Hou, Adv. Mater., 2018, 30, 1707170 .

140 A. Facchetti, Mater. Today, 2013, 16, 123.

141 H. Benten, D. Mori, H. Ohkita and S. Ito, J. Mater. Chem. A, 2016, 4, 5340.

142 C. R. McNeill, S. Westenhoff, C. Groves, R. H. Friend and N. C. Greenham, J. Phys. Chem. C, 2007, 111, 19153.

143 S. Westenhoff, I. A. Howard, J. M. Hodgkiss, K. R. Kirov, H. A. Bronstein, C. K. Williams, N. C. Greenham and R. H. Friend, J. Am. Chem. Soc., 2008, 130, 13653.

144 J. M. Hodgkiss, A. R. Campbell, R. A. Marsh, A. Rao, S. AlbertSeifried and R. H. Friend, Phys. Rev. Lett., 2010, 104, 177701.

145 J. R. Moore, S. Albert-Seifried, A. Rao, S. Massip, B. Watts, D. J. Morgan, R. H. Friend, C. R. McNeill and H. Sirringhaus, Adv. Energy Mater., 2011, 1, 230.
146 H. Ohkita, J. Kosaka, J. Guo, H. Benten and S. Ito, J. Photonics Energy, 2011, 1, 011118.

147 M. Schubert, B. A. Collins, H. Mangold, I. A. Howard, W. Schindler, K. Vandewal, S. Roland, J. Behrends, F. Kraffert, R. Steyrleuthner, Z. Chen, K. Fostiropoulos, R. Bittl, A. Salleo, A. Facchetti, F. Laquai, H. W. Ade and D. Neher, Adv. Funct. Mater., 2014, 24, 4068.

148 D. Mori, H. Benten, H. Ohkita and S. Ito, Adv. Energy Mater., 2015, 5, 1500304.

149 B. R. Gautam, C. Lee, R. Younts, W. Lee, E. Danilov, B. J. Kim and K. Gundogdu, ACS Appl. Mater. Interfaces, 2015, 7, 27586.

150 H. Yan, Z. Chen, Y. Zheng, C. Newman, J. R. Quinn, F. Dotz, M. Kastler and A. Facchetti, Nature, 2009, 457, 679.

151 L. Zhu, W. Zhong, C. Qiu, B. Lyu, Z. Zhou, M. Zhang, J. Song, J. Xu, J. Wang, J. Ali, W. Feng, Z. Shi, X. Gu, L. Ying, Y. Zhang, F. Liu, Adv. Mater., 2019, 31, 1902899.

152 Y. Hwang, B. A. E. Courtright, A. S. Ferreira, S. H. Tolbert and S. A. Jenekhe, Adv. Mater., 2015, 27, 4578.

153 Y. Hwang, T. Earmme, B. A. E. Courtright, F. N. Eberle and S. A. Jenekhe, J. Am. Chem. Soc., 2015, 137, 4424.

154 M. Schubert, D. Dolfen, J. Frisch, S. Roland, R. Steyrleuthner, B. Stiller, Z. Chen, U. Scherf, N. Koch, A. Facchetti and D. Neher, Adv. Energy Mater., 2012, 2, 369.

155 L. Koster, V. Mihailetchi and P. Blom, Appl. Phys. Lett., 2006, 88, 052104.

156 C. Groves and N. C. Greenham, Phys. Rev. B, 2008, 78, 155205.

157 M. C. Heiber, C. Baumbach, V. Dyakonov and C. Deibel, Phys. Rev. Lett., 2015, 114, 136602.

158 V. Coropceanu and J. L. Brédas, J. Phys. Chem. C, 2017, 121, 24954.

159 G. Juška, K. Genevičius, N. Nekrašas, G. Sliaužys and R. Österbacka, Appl. Phys. Lett., 2009, 95, 013303.

160 K. Tvingstedt, K. Vandewal, A. Gadisa, F. Zhang, J. Manca and O. Inganäs, J. Am. Chem. Soc., 2009, 131, 11819.

161 S. Yamamoto, H. Ohkita, H. Benten and S. Ito, J. Phys. Chem. C, 2012, 116, 14804.

162 T. M. Burke, S. Sweetnam, K. Vandewal and M. D. McGehee, Adv. Energy Mater., 2015, 5, 1500123.

163 Y. Liu, K. Zojer, B. Lassen, J. K. Hansen, H. G. Rubahn and M. Madsen, J. Phys. Chem. C, 2015, 119, 26588. 
164 A. Rao, P. C. Y. Chow, S. Gelinas, C. W. Schlenker, C. Li, H. Yip, A. K. Y. Jen, D. S. Ginger and R. H. Friend, Nature, 2013, 500, 435.

165 P. C. Y. Chow, S. Gelinas, A. Rao and R. H. Friend, J. Am. Chem. Soc., 2014, 136, 3424.

166 A. Armin, J. R. Durrant and S. Shoaee, J. Phys. Chem. C, 2017, 121, 13969.

167 F. C. Jamieson, E. B. Domingo, T. McCarthy-Ward, M. Heeney, N. Stingelin and J. R. Durrant, Chem. Sci., 2012, 3, 485.

168 S. Sweetnam, K. R. Graham, G. O. N. Ndjawa, T. Heumueller, J. A. Bartelt, T. M. Burke, W. Li, W. You, A. Amassian and M. D. McGehee, J. Am. Chem. Soc., 2014, 136, 14078.

169 J. A. Bartelt, D. Lam, T. M. Burke, S. M. Sweetnam and M. D. McGehee, Adv. Energy Mater., 2015, 5, 1500577.

170 J. K. Gallaher, S. K. K. Prasad, M. A. Uddin, T. Kim, J. Y. Kim, H. Y. Woo and J. M. Hodgkiss, Energy Environ. Sci., 2015, 8, 2713.

171 C. Groves, Energy Environ. Sci., 2013, 6, 1546.

172 L. Yang, L. Yan and W. You, J. Phys. Chem. Lett., 2013, 4, 1802.

173 L. Lu, M. A. Kelly, W. You and L. Yu, Nat. Photonics, 2015, 9, 491.

174 Y. Yang, W. Chen, L. Dou, W. Chang, H. Duan, B. Bob, G. Li and Y. Yang, Nat. Photonics, 2015, 9, 190.

175 Q. An, F. Zhang, J. Zhang, W. Tang, Z. Deng and B. Hu, Energy Environ. Sci., 2016, 9, 281.

176 S. Honda, T. Nogami, H. Ohkita, H. Benten and S. Ito, ACS Appl. Mater. Interfaces, 2009, 1, 804.

177 S. Honda, H. Ohkita, H. Benten and S. Ito, Chem. Commun., 2010, 46, 6596

178 S. Honda, H. Ohkita, H. Benten and S. Ito, Adv. Energy Mater., 2011, 1, 588.

179 H. Xu, T. Wada, H. Ohkita, H. Benten and S. Ito, Electrochim. Acta, 2013, 100, 214.

180 H. Xu, H. Ohkita, T. Hirata, H. Benten and S. Ito, Polymer, 2014, 55, 2856.

181 H. Xu, H. Ohkita, H. Benten and S. Ito, Jpn. J. Appl. Phys., 2014, 53, $01 \mathrm{AB} 10$.

182 Y. Wang, B. Zheng, Y. Tamai, H. Ohkita, H. Benten and S. Ito, J. Electrochem. Soc., 2014, 161, D3093.
183 Y. Wang, H. Ohkita, H. Benten and S. Ito, Trans. Mater. Res. Soc. Jpn., 2014, 39, 439.

184 H. Xu, H. Ohkita, Y. Tamai, H. Benten and S. Ito, Adv. Mater., 2015, 27, 5868.

185 Y. Wang, H. Ohkita, H. Benten and S. Ito, Phys. Chem. Chem. Phys., 2015, 17, 27217.

186 H. Benten, T. Nishida, D. Mori, H. Xu, H. Ohkita and S. Ito, Energy Environ. Sci., 2016, 9, 135.

187 H. Benten, T. Nishida, D. Mori, H. Ohkita and S. Ito, J. Photopolym. Sci. Technol., 2016, 29, 537.

188 Y. Wang, J. Chen, H. D. Kim, B. Wang, R. Iriguchi and H. Ohkita, Front. Energy Res., 2018, 6, 113.

189 Y. Wang, H. D. Kim, B. Wang and H. Ohkita, J. Photopolym. Sci. Technol., 2018, 31, 177.

190 H. D. Kim, R. Shimizu and H. Ohkita, Chem. Lett., 2018, 47, 1059.

191 Y. Wang, T. Wang, J. Chen, H. Do Kim, P. Gao, B. Wang, R. Iriguchi and H. Ohkita, Dyes Pig., 2018, 158, 213.

192 H. Xu, T. Wada, H. Ohkita, H. Benten and S. Ito, Sci. Rep., 2015, 5, 9321.

193 Z. Tan, K. Johnson, Y. Vaynzof, A. A. Bakulin, L. Chua, P. K. H. Ho and R. H. Friend, Adv. Mater., 2013, 25, 4131.

194 S. Izawa, K. Nakano, K. Suzuki, K. Hashimoto and K. Tajima, Adv. Mater., 2015, 27, 3025.

195 Q. Wei, K. Tajima and K. Hashimoto, ACS Appl. Mater. Interfaces, 2009, 1, 1865.

196 Q. Wei, K. Tajima, Y. Tong, S. Ye and K. Hashimoto, J. Am. Chem. Soc., 2009, 131, 17597.

197 N. Gasparini, X. Jiao, T. Heumueller, D. Baran, G. J. Matt, S. Fladischer, E. Spiecker, H. Ade, C. J. Brabec and T. Ameri, Nat. Energy, 2016, 1, 16118.

198 D. Baran, R. S. Ashraf, D. A. Hanifi, M. Abdelsamie, N. Gasparini, J. A. Rohr, S. Holliday, A. Wadsworth, S. Lockett, M. Neophytou, C. J. M. Emmott, J. Nelson, C. J. Brabec, A. Amassian, A. Salleo, T. Kirchartz, J. R. Durrant and I. McCulloch, Nat. Mater., 2017, 16, 363.

199 B. A. Gregg, J. Phys. Chem. Lett., 2011, 2, 3013.

200 D. Bartesaghi, I. del C. Pérez, J. Kniepert, S. Roland, M. Turbiez, D. Neher and L. J. A. Koster, Nat. Commun., 2015, 6, 7083. 
201 D. Neher, J. Kniepert, A. Elimelech and L. J. A. Koster, Sci. Rep., 2016, 6, 24861.

202 U. Würfel, D. Neher, A. Spies and S. Albrecht, Nat. Commun., $2015,6,6951$.

203 J. C. Blakesley and D. Neher, Phys. Rev. B, 2011, 84, 075210.

204 M. Azzouzi, J. Yan, T. Kirchartz, K. Liu, J. Wang, H. Wu and J. Nelson, Phys. Rev. X, 2018, 8, 031055.

205 D. Baran, T. Kirchartz, S. Wheeler, S. Dimitrov, M. Abdelsamie, J. Gorman, R. S. Ashraf, S. Holliday, A. Wadsworth, N. Gasparini, P. Kaienburg, H. Yan, A. Amassian, C. J. Brabec, J. R. Durrant and I. McCulloch, Energy Environ. Sci., 2016, 9, 3783.

206 W. Zhao, D. Qian, S. Zhang, S. Li, O. Inganäs, F. Gao and J. Hou, Adv. Mater., 2016, 28, 4734.

207 M. E. Ziffer, S. B. Jo, H. Zhong, L. Ye, H. Liu, F. Lin, J. Zhang, X. Li, H. W. Ade, A. K. Y. Jen and D. S. Ginger, J. Am. Chem. Soc., 2018, 140, 9996.

208 X. Liu, X. Du, J. Wang, C. Duan, X. Tang, T. Heumueller, G. Liu, Y. Li, Z. Wang, J. Wang, F. Liu, N. Li, C. J. Brabec, F. Huang and Y. Cao, Adv. Energy Mater., 2018, 8, 1801699.

209 J. Yuan, T. Huang, P. Cheng, Y. Zou, H. Zhang, J. L. Yang, S. Chang, Z. Zhang, W. Huang, R. Wang, D. Meng, F. Gao and Y. Yang, Nat. Commun., 2019, 10, 570.

210 Y. Wang, Y. Wang, L. Zhu, H. Liu, J. Fang, X. Guo, F. Liu, Z. Tang, M. Zhang and Y. Li, Energy Environ. Sci., 2020, 13, 1309.

211 F. D. Eisner, M. Azzouzi, Z. Fei, X. Hou, T. D. Anthopoulos, T. S. Dennis, M. Heeney and J. Nelson, J. Am. Chem. Soc., 2019, 141, 6362.

212 G. D'Avino, L. Muccioli, Y. Olivier and D. Beljonne, J. Phys. Chem. Lett., 2016, 7, 536.

213 X. K. Chen, V. Coropceanu and J. L. Brédas, Nat. Commun., 2018, 9, 5295.

214 D. Qian, Z. Zheng, H. Yao, W. Tress, T. R. Hopper, S. Chen, S. Li, J. Liu, S. Chen, J. Zhang, X. K. Liu, B. Gao, L. Ouyang, Y. Jin, G. Pozina, I. A. Buyanova, W. M. Chen, O. Inganäs, V. Coropceanu, J. L. Brédas, H. Yan, J. Hou, F. Zhang, A. A. Bakulin and F. Gao, Nat. Mater., 2018, 17, 703.

215 T. Sato, R. Hayashi, N. Haruta and Y. J. Pu, Sci. Rep., 2017, 7, 4820. 